\title{
Drug Use and the Risk of Motor Vehicle Collision in Adults 65 Years of Age and Older
}

\author{
Toni Marie Rudisill
}

Follow this and additional works at: https://researchrepository.wvu.edu/etd

\section{Recommended Citation}

Rudisill, Toni Marie, "Drug Use and the Risk of Motor Vehicle Collision in Adults 65 Years of Age and Older" (2015). Graduate Theses, Dissertations, and Problem Reports. 6539.

https://researchrepository.wvu.edu/etd/6539

This Dissertation is protected by copyright and/or related rights. It has been brought to you by the The Research Repository @ WVU with permission from the rights-holder(s). You are free to use this Dissertation in any way that is permitted by the copyright and related rights legislation that applies to your use. For other uses you must obtain permission from the rights-holder(s) directly, unless additional rights are indicated by a Creative Commons license in the record and/ or on the work itself. This Dissertation has been accepted for inclusion in WVU Graduate Theses, Dissertations, and Problem Reports collection by an authorized administrator of The Research Repository @ WVU.

For more information, please contact researchrepository@mail.wvu.edu. 
Drug Use and the Risk of Motor Vehicle Collision in Adults 65 Years of Age and Older

Toni Marie Rudisill, MS

Dissertation submitted

to the School of Public Health

at West Virginia University

in partial fulfillment of the requirements for the degree of

Doctor of Philosophy in

Epidemiology

Motao Zhu, MD, MS, PhD Chair

Danielle Davidov, PhD

D. Leann Long, PhD

Usha Sambamoorthi, PhD

Marie Abate, PharmD

Vincent Delagarza, MD

Department of Epidemiology

Morgantown, West Virginia

2015

Keywords: Collisions, Motor vehicle, Seniors, Epidemiology, Drugs

Copyright 2015 Toni Marie Rudisill, MS 


\title{
ABSTRACT \\ Drug Use and the Risk of Motor Vehicle Collision in Adults 65 Years of Age and Older
}

\author{
Toni Marie Rudisill, MS
}

Introduction: The population of adults 65 years of age and older is growing at an exponential rate in the United States. The current generation of older adults is more mobile and driving much later in life compared to previous cohorts. While the current generation of older adults is living longer, they are not necessarily healthier. Older adults consume roughly $30 \%$ of all written prescriptions, are the largest consumers of over-the-counter medications, and report a higher prevalence of lifetime licit and illicit drug use compared to previous generations. There is concern that many older adults will drive while taking drugs - either illicit or licit - that will interfere with their ability to safely operate a motor vehicle. Licit and illicit drug use is largely understudied in this population of drivers, particularly in the United States.

Methods: This study employed a systematic review to discern which individual licit drugs were associated with increased risk/odds of motor vehicle collision, a cross-sectional analysis of a national traffic fatality database to discern which types of licit and/or illicit drugs and combinations were most prevalent among fatally injured senior drivers compared to middle-aged drivers, and a case-crossover study using electronic medical records to assess the risk that individual licit drugs pose to drivers 65 years of age and older in West Virginia who were admitted to medical treatment following a motor vehicle collision.

Results: Among the 53 specific medications investigated by the 27 studies included in the systematic review, 15 medications (28.3\%) were associated with an increased risk of motor vehicle collision. The medications that were associated with an increased risk of collision were: Buprenorphine, Codeine, Dihydrocodeine, Methadone, Tramadol, Levocitirizine, Diazepam, Flunitrazepam, Flurazepam, Lorazepam, Temazepam, Triazolam, Carisoprodol, Zolpidem, and Zopiclone. In the cross-sectional analysis, licit and illicit drug use among senior drivers occurred in 17.8\% of those tested. Among drugpositive senior drivers, benzodiazepines and narcotics were frequent. The rates of testing positive for any drug, multiple drugs, combined drug and alcohol, and alcohol use alone among seniors were $47 \%$ (Rate ratio, $\mathrm{RR}=0.53,95 \% \mathrm{CI} 0.50,0.57), 57 \%(\mathrm{RR}=0.43,95 \% \mathrm{CI} 0.39,0.48), 88 \%(\mathrm{RR}=0.12,95 \% \mathrm{CI} 0.10$, $0.15)$ and $79 \%(\mathrm{RR}=0.21,95 \%$ CI $0.19,0.24)$ less, respectively, compared to middle-aged drivers. In the case-crossover study, few drivers tested positive for drugs. Of those testing positive, benzodiazepines and opiates were the most common. After adjusting for the number of medications a driver was consuming during case and control periods, cases consuming Tramadol (Odds ratio, OR: 11.41; 95\% CI 1.27, 102.15), were at a significantly increased risk of motor vehicle collision if they consumed these medications 14 days before the collision compared to control periods. Numerous other medications, including Clopidogrel, Gabapentin, Citalopram, Insulin, Hydrochlorothiazide, Metoprolol, Zolpidem, and Nitroglycerine, were trending towards being associated with motor vehicle collision, but were not found statistically significant.

Conclusions: The findings of this research pose numerous clinical and research implications. As many of the licit drugs included in this analysis are widely prescribed, patients should be informed that their driving ability may be affected. Further exploration into this area is research is necessary as older adults are choosing to maintain their mobility later in life. 


\section{DEDICATION}

I dedicate my doctoral dissertation to my parents, John and Annette Pietrolaj, and my loving husband, Brandon Rudisill. Without all your love and support over the years, this dissertation would not have been possible.

\section{ACKNOWLEDGEMENTS}

There is the old adage, 'it takes a village to raise a child,' and I believe this holds true with a doctoral education - it takes a village to produce a $\mathrm{PhD}$. There are many, many people who greatly influenced my life and education from the beginning making this lifelong dream become a reality. First, I would like to extend my most heartfelt gratitude to my parents, John and Annette Pietrolaj, who fostered a loving environment throughout my youth. I love you both and am grateful for everything you have done. Second, I would like to extend my gratitude to my husband, Brandon. I cannot thank you enough for all your love, patience, and support. I am sorry that I often spent more time in front of the computer than with you or if I was grouchy at times during this LONG process. I am so grateful to have you in my life. You're the best!

I would also like to extend my sincere appreciation to my mentor, Dr. Motao Zhu. I sincerely appreciate your time, guidance, and patience over the past few years. It has been a pleasure to work with you and I am so thankful that you agreed to oversee my research. I would also like to extend my gratitude towards my dissertation committee members, Dr. Danielle Davidov, Dr. Leann Long, Dr. Usha Sambamoorthi, Dr. Marie Abate, and Dr. Vincent Delagarza. Thank you for your time, guidance, and helpful comments-you are all wonderful to work with!

I would also like to extend my gratitude to West Virginia University and the faculty and staff of the School of Public Health. Thank you for accepting me into your community and program and for providing guidance and wisdom. I would also like to especially thank Dr. Keith Zullig. Words cannot express my gratitude towards you. Without your encouragement and support, this dissertation would never have happened.

A special, heartfelt thank you goes to the current and former students enrolled in the Public Health Sciences program, but especially to members of my cohort: Z Haile, Courtney Pilkerton, Meagan Stabler, and Yahya Alamir. You are all such wonderful, intelligent people. You all made some of those long hours studying more bearable with your wit and humor. I am honored to have worked with all of you. 


\section{TABLE OF CONTENTS}

\section{Chapter 1: Literature Review}

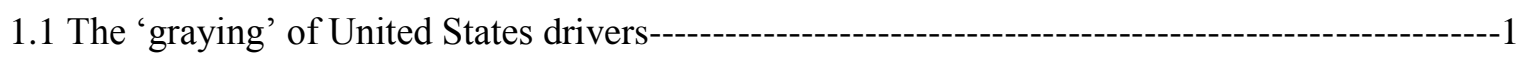

1.2 The importance of mobility on health and well-being ---

1.3 Crash risks among older adult drivers ------------------------------------------------5

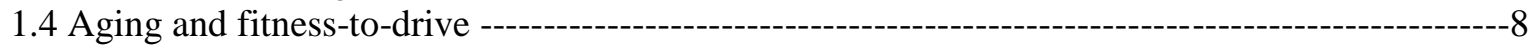

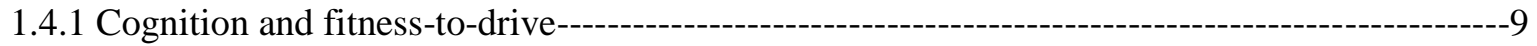

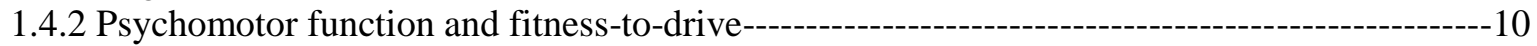

1.4.3 Vision and fitness-to-drive--------

1.4.4 Hearing and fitness-to-drive-------

1.4.5 Physical limitations and fitness-to-drive----------12

1.5 Medical conditions associated with motor vehicle collision risk-------------------------------------12

1.6 Licit drugs and motor vehicle collision risk---on

1.6.1 The effects of licit drugs on motor vehicle collision risk--------- 18

1.6.2 The effects of licit drugs on actual driving ability--------------------------- 23

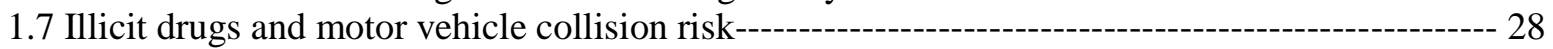

1.7.1 The effects of individual illicit drugs on motor vehicle collision risk-----------------28

1.7.2 The effects of illicit drugs on actual driving ability----------29

1.8 Challenges of studying the association between drug use and driving ability--------------------30

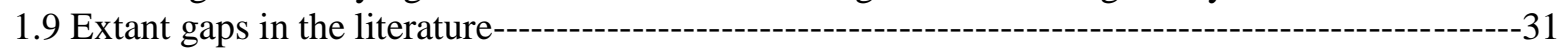

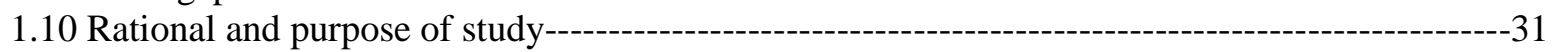

\section{Chapter 2: Manuscript 1}

Medication use and the risk of motor vehicle collisions among licensed drivers: A systematic review

2.1 Introduction----------------------------------------------------------------------------34

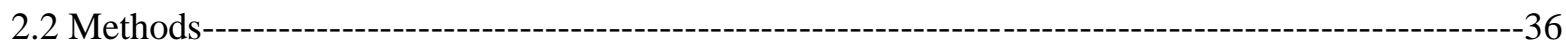

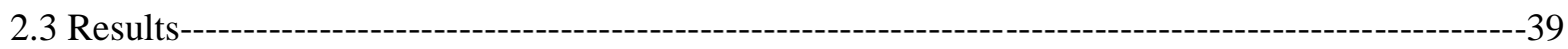

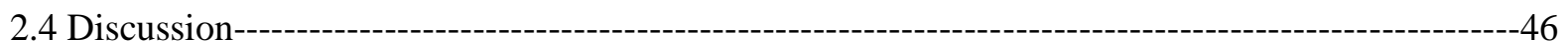

Chapter 3: Manuscript 2

Licit and illicit drug use among senior drivers killed in U.S. motor vehicle collisions, 2008-2012

3.1 Introduction-----

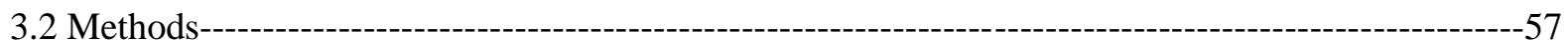

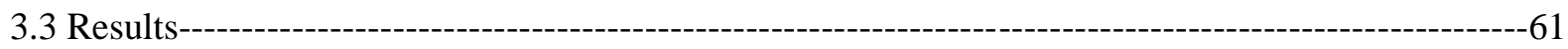

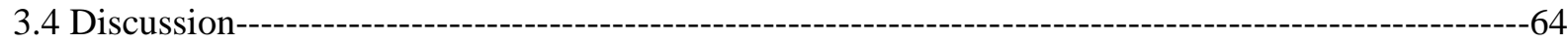

Chapter 4: Manuscript 3

Medication use and the risk of motor vehicle collision in West Virginia drivers 65 years of age and older: a case-crossover study

4.1 Introduction----

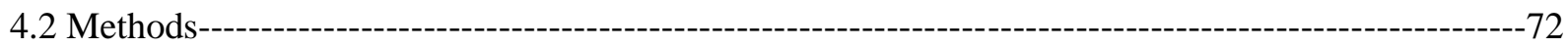

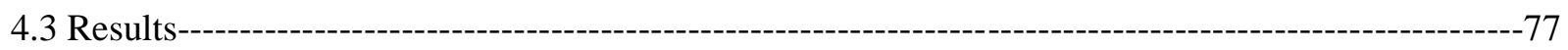

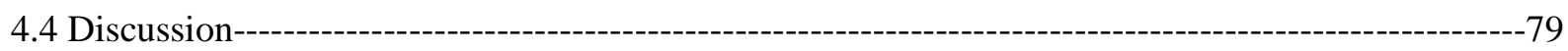

\section{Chapter 5: Discussion}

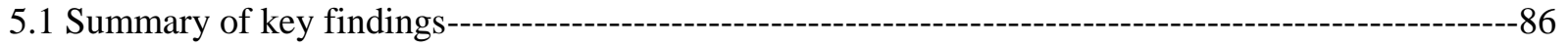

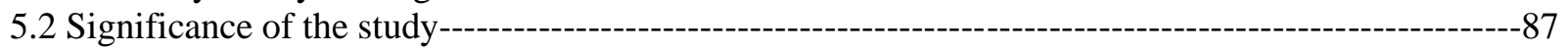

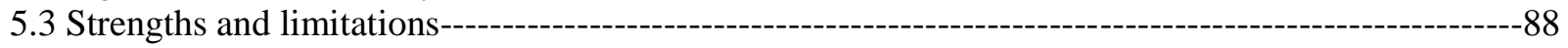

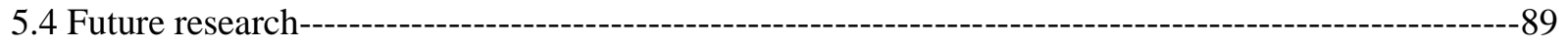




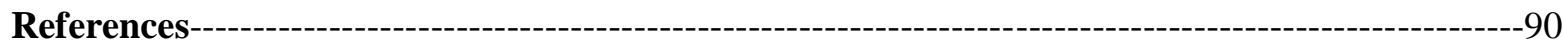

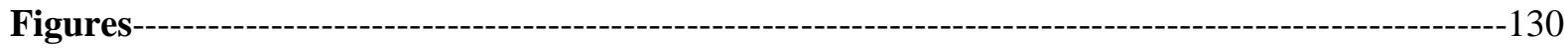

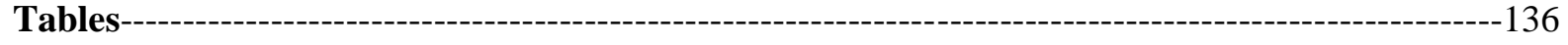




\section{Chapter 1: Literature review}

\subsection{The 'graying' of United States drivers}

Due to increases in both life expectancy and fertility post-World War II, the United States (U.S.) is experiencing an unprecedented demographic shift with the number of adults 65 years of age and older increasing faster than any other age group (Centers for Disease Control and Prevention 2003). This translates into a one million person per year increase in the 65 and older age group as projected through 2030 (Centers for Disease Control and Prevention 2003). The current generation of older adults is living their lives quite differently than previous cohorts. Quality of life is highly valued (Rappaport 2009), and compared to their predecessors, the older adults of today are increasingly mobile and heavily engaged in their personal and professional lives (Becton, Walker et al. 2014). In a 2010 survey of 225 older adults, most reported that they planned to continue working later in life and intended to increase their travel and civic engagements (MaloneBeach and Langeland 2010). Overall, the current generation of older adults is wealthier, better educated, and more likely than their predecessors to remain employed (Topiwala, Patel et al. 2014). Between 1977 and 2007, the number of adults who continued to work past 75 years of age increased 172\% (Tang, Choi et al. 2013), which has been attributed to gaps in health insurance, educational attainment burdens, and decreases in pension plans (Mermin, Johnson et al. 2007).

The current demographic shift is not without consequence. Despite higher socio-economic status and advancements in healthcare, the current generation of older adults is not markedly healthier than their predecessors, though they are living longer (Martin, Freedman et al. 2009). Consequently, it is projected that this generation of older adults will experience increases in chronic disease and complex medical conditions which will burden the healthcare system (Dall, Gallo et al. 2013). It is projected that this demographic shift will cause a 14\% increase in demand for primary care physicians (Dall, Gallo et al. 2013) along with increases in the length of hospital stays and number of hospital admissions (Pallin, Allen et al. 2013; Pallin, Espinola et al. 2014). Emergency department capacity and hospital admission rates will need to increase $10 \%$ and $23 \%$, respectively, faster than the population grows in order to keep 
up with demand (Pallin, Allen et al. 2013; Pallin, Espinola et al. 2014). Therefore, meeting the healthcare demands of this generation of older adults is one of the largest public health hurdles to date.

Besides the increased demands on the healthcare system, the demographic shift has also placed additional strain on the transportation system. Because older adults are continuing to work and travel, they are also driving later in life compared to previous generations. Agencies, such as the U.S. Department of Transportation, have been proactively working to develop a national agenda to promote safe mobility due to increasing number of older drivers (Stutts 2005). These measures have included ways to make roads safer through engineering pursuits, to promote safer drivers thru stricter relicensing measures, and encouraging the motor vehicle industry to create safer vehicles (Oxley, Langford et al. 2010). Such agencies acknowledge that driving is inherent to American culture and many depend solely on personal transportation for their mobility. Reliance on public transportation in the U.S. is declining among older adults while their number of trips taken and miles traveled continue to increase (Rosenbloom 2003). Approximately $79 \%$ of older adults reside in rural or suburban areas (Rosenbloom 2003), causing many of these individuals to rely on their personal vehicles for transportation purposes (Schwarzlose, Mjelde et al. 2014).

\subsection{The importance of mobility on health and well-being}

Mobility is broadly defined as an individual's ability to move about their environment (Webber, Porter et al. 2010). While individuals can navigate their environment various ways, such as walking, taking public transportation, riding as a passenger, or driving, most Americans equate mobility with driving a personal vehicle (King, Meuser et al. 2011). It is widely accepted that driving is associated with freedom and independence. Dickerson et al investigated the importance of instrumental activities of daily living (IADL) in a group of 30 seniors who were returning home after receiving extensive rehabilitation; when asked to rate the importance, being able to drive and manage medications, respectively, were the first and second most valued tasks (Dickerson, Reistetter et al. 2013). A significant body of literature 
exists regarding the importance of mobility and its effect on individual health and well-being. Driving cessation has been linked to various negative health outcomes, which can be categorized as physical/tangible measures, including unmet leisure needs (Bonnel 1999; Marottoli, de Leon et al. 2000; Haustein and Siren 2014), reduced social networks (Mezuk and Rebok 2008; Pekmezaris, Kozikowski et al. 2013), even mortality (Edwards, Perkins et al. 2009), or poorer emotional/mental health (Johnson 1999; Fonda, Wallace et al. 2001; Ragland, Satariano et al. 2005; Gagliardi, Marcellini et al. 2010; King, Meuser et al. 2011; Al-Hassani and Alotaibi 2014).

Three studies investigated the effects of driving cessation on unmet leisure needs. A qualitative study conducted by Bonnell et al investigated the effects of driving cessation on 15 women through focus group sessions (Bonnel 1999). Driving cessation is an important issue to older women's health as more women live alone (41\%) as age increases (Bonnel 1999). Virtually all participants reported that they gave up social outings, missed doctor's appointments, church and family gatherings, meals, volunteering opportunities, and leisure activities (such as shopping) when they ceased driving. Many sought out other means of transport which included walking, relying on family and friends, services vans, and public transportation (Bonnel 1999). A study by Haustein and Siren investigated how important a driving license is for seniors to fulfill their mobility needs (Haustein and Siren 2014). Data were collected in 2012 from 863 individuals born in either 1939 or 1940 via telephone interviews. The study compared those that were currently licensed, had never been licensed, or gave up their licenses. Both never having a license and having given up a license significantly affected unmet mobility needs (Haustein and Siren 2014). A study by Marotolli et al was designed to test the hypothesis that driving cessation leads to a decline in out-of-home activity levels (Marottoli, de Leon et al. 2000). Participants came from the New Haven Established Populations for Epidemiologic Studies of the Elderly (EPESE) cohort. After adjusting for potential demographic and health-related variables, driving cessation was significantly associated with decreased out-of-home activity levels (Marottoli, de Leon et al. 2000). 
Two studies have investigated the effects of driving cessation on social isolation. A study by Mezuk et al evaluated the impact of driving cessation on social integration and perceived support from relatives and friends among older adults (Mezuk and Rebok 2008). Data came from the population-based Baltimore Epidemiologic Catchment Area Study. Social integration and perceived social support were assessed at two interviews staged over the course of 13 years. The use of public transportation was investigated as a potential mediator. Driving cessation was significantly associated with a reduced number/network of friends that was not mediated by the use of public transportation (Mezuk and Rebok 2008). A study by Pekmezarius et al surveyed 1,151 seniors to examine the needs of seniors living in a large suburban community across two counties in New York State (Pekmezaris, Kozikowski et al. 2013). Findings revealed that seniors over 85 years of age and those who do not drive were more socially isolated even though public transportation was readily available (Pekmezaris, Kozikowski et al. 2013).

One study investigated if driving cessation was a predictor of mortality (Edwards, Perkins et al. 2009). In a 2000-2004 prospective study of 660, community-dwelling, adults over 63 years of age, being a non-driver at follow up was significant predictor of three year survival (Edwards, Perkins et al. 2009).

Six studies investigated the effects of driving cessation on mental health. In a study by AlHassani et al a questionnaire was sent to 114 community dwelling seniors regarding their driving status and mental health. Results showed that for non-drivers, driving cessation created feelings of dependency and loss of control (Al-Hassani and Alotaibi 2014). A study by Gagliardi et al investigated association between mobility and positive well-being in two sample populations of older adults from Germany $(\mathrm{n}=$ 1518) and Italy $(n=600)$ who were part of the MOBILATE European cohort study. Results showed that in both countries the driving car is an important predictor of well-being, mobility, health, and life satisfaction (Gagliardi, Marcellini et al. 2010). A study by Johnson investigated the effects of licensure forfeiture in a convenience sample of 285 urban dwelling adults who had stopped driving within the past year in an undisclosed city in the Western U.S. Approximately $80 \%$ of respondents reported feelings of loneliness after drivers license forfeiture and felt their mobility was compromised even though public 
transportation was available (Johnson 1999). A study by King et al investigated attitudes, beliefs, values, and emotions associated with real or anticipated changes in mobility status in a convenience sample of 30 adults over 57 years of age in an undisclosed Midwestern city (King, Meuser et al. 2011). Participants were separated into focus groups by age and disability status. Fear of dependence was an important feeling in all groups. Many said they experienced an array of emotions such as devastation, resentment, anger, frustration, loneliness, and isolation after they could no longer drive (King, Meuser et al. 2011). A study by Fonda et al investigated whether changes in driving patterns (i.e. limiting driving distances or driving cessation) were associated with depressive symptoms among older adults (Fonda, Wallace et al. 2001). The participants in this study $(n=4,102)$ were individuals from the Asset and Health Dynamics among the Oldest Old (AHEAD) cohort. Results showed that respondents who stopped driving had greater risk of worsening depressive symptoms. Interestingly, having a spouse available to drive those who no longer drove did not mediate this effect (Fonda, Wallace et al. 2001). A study by Ragland et al evaluated clinical depression in former drivers compared with active drivers. Participants were part of a cohort of 1,953 residents of Sonoma County, California, aged 55 years and older, as part of a communitybased study of aging and physical performance (Ragland, Satariano et al. 2005). Results showed that after controlling for age, sex, education, health, and marital status, former drivers reported higher levels of depression than did active drivers. Increased depression was substantially higher in men that no longer drove compared to women who no longer drove (Ragland, Satariano et al. 2005).

Findings from these studies show how important driving is to an individual's health and wellbeing. While the ability to drive is highly revered among older adults, findings from the Bonnell et al study report that pressure from adult children to cease driving, decreases in reflexes, fear of liability, car repair costs, and safety concerns are some of the reasons why some seniors choose to no longer drive (Bonnel 1999). Respondents from the Johnson study reported that mandatory license revocation or pressure from family and friends were the primary reasons for driver's license forfeiture (Johnson 1999).

\subsection{Crash risks among older adult drivers}


While maintaining mobility is crucial for older adults' health and well-being, driving cessation is unavoidable for many. Deciding when to cease driving is not always clear. Due to the increasing number of older adult drivers, the crash risk that older drivers pose to themselves and others has generated much research interest. To date, this issue has been investigated numerous ways, but can generally be grouped into the following categories: 1) trend analyses of older driver crash rates, 2) comparisons of fatal and non-fatal crash rates between different age groups of drivers, 3) hospitalization or injury rates of elderly drivers, passengers, and/or pedestrians, and 4) where and how older driver collisions occur.

As for trend analyses, one study has been conducted to date (Cheung and McCartt 2011). Cheung et al investigated fatal and non-fatal crash rates of older drivers using crash data from 13 states between 1997 and 2008. The findings from this analysis reveal that both fatal and non-fatal crash rates have declined considerably in the U.S. among older adult drivers during this 12 -year period (Cheung and McCartt 2011).

While crash rates appear to be declining among older adult drivers, they are not necessarily low. In 2011, the National Highway Traffic Safety Administration reports that $17 \%$ of all traffic fatalities were among adults aged 65 years and older (National Highway Traffic Safety Administration 2013). Three studies have been conducted in the U.S. using national crash data to compare rates between different age groups (Massie, Campbell et al. 1995; Li, Braver et al. 2003; Williams and Shabanova 2003). Li et al compared older drivers age 60 and over, 16-19, 20-29, and 30-59 year old drivers. Results showed that older drivers had much higher death rates per vehicle miles traveled compared to other age groups ( $\mathrm{Li}$, Braver et al. 2003). Massie et al compared fatal crash involvements per million miles traveled in 16-19, 20-24, 25-29, 30-34, 35-39, 40-44, 45-49, 50-54, 55-59, 60-64, 65-69, and $\geq 70$ year old drivers. Findings show that the oldest drivers had the highest fatal crash involvement rates compared to all other age groups (Massie, Campbell et al. 1995). Williams et al compared the rates of drivers in fatal crashes involving one or two passenger vehicles per 100,000 license holders, by driver age group and gender. The authors also investigated driver fault. Findings showed that the oldest drivers were more likely than any other 
group to be responsible for their fatal crashes (Williams and Shabanova 2003). The findings from the U.S. studies are similar to those conducted in Australia (Ryan, Legge et al. 1998; Keall and Frith 2004; Keall and Frith 2006; Langford, Bohensky et al. 2008; Langford, Koppel et al. 2008; Langford, Charlton et al. 2013) and Canada (Lefrancois and D'Amours 1997; Myrick 2001). The findings from the Australian and Canadian studies have also found drivers over 70 to be involved in more fatal and nonfatal collisions per vehicle miles traveled and drivers who drive less miles per year to be at heightened crash risk (Lefrancois and D'Amours 1997; Ryan, Legge et al. 1998; Myrick 2001; Keall and Frith 2004; Keall and Frith 2006; Langford, Bohensky et al. 2008; Langford, Koppel et al. 2008; Langford, Charlton et al. 2013).

As for injury or hospitalization rates of older adult drivers and their passengers, three studies have investigated these topics (Tavris, Kuhn et al. 2001; Braver and Trempel 2004; Dellinger, Kresnow et al. 2004). Tavris et al studied the differences in gender and hospitalization rates for drivers and passengers involved in motor vehicle collisions in Wisconsin using administrative claims data. Results showed that males were hospitalized 33\% more than females and that hospitalizations of drivers increased significantly in men starting at age 70. Passenger injury rates were highest in women over 85 years of age (Tavris, Kuhn et al. 2001). Braver et al used insurance data to calculate the injury rates per driver for various road users. Results of this analysis showed that older drivers pose a $152 \%$ increased risk of fatality to their passengers who also tend to be aged (Braver and Trempel 2004). Dellinger et al assessed the risk of fatality and non-fatal injury to other road users (i.e. cyclists, pedestrians, etc) by drivers over 65 years of age and compared these rates to various other age groups. The findings showed that drivers over 74 years of age posed more risk of death to others and that drivers $\geq 85$ years of age posed the most risk of injury to other road users per million miles driven (Dellinger, Kresnow et al. 2004).

Four studies have investigated which type of road conditions or driving situations pose the most risk to older adult driver safety (McGwin and Brown 1999; Bedard 2000; Laing 2003; Surapaneni 2006). Bedard et al studied every fatal traffic incident from 1975-1998 using U.S. crash data and found that 
drivers over 80 years of age were eight times at risk for committing driving errors associated with road signs or warnings, six times the risk of collision for entering or exiting traffic flow, and seven times are risk to commit errors at intersections compared to drivers aged 40-49 (Bedard 2000). Laing et al investigated collisions in British Columbia by age between 1996 and 2000 and found that drivers over 65 years of age have higher collision rates at intersections (Laing 2003). Mcgwin et al studied traffic collisions by different age groups in Alabama during 1996. Findings from their analysis showed that older drivers experienced more collisions at intersections or during turns or lane changes and often failed to yield the right of way or heed traffic control devices compared to other age groups (McGwin and Brown 1999). Surapaneni investigated collisions of older adult drivers ( $\geq 75$ years) compared to various other age groups in Clark County, Nevada. Based on rates per million vehicle miles traveled, older drivers had higher rates of collisions involving turns, failure to yield, and lane changes compared to other ages (Surapaneni 2006).

As evident from the reviewed literature, older adult drivers do appear to be at higher risk of collision compared to younger age groups, especially when taking the amount they drive (i.e. miles driven daily, weekly, etc) into account. Older drivers seem to be at higher risk of hospitalization or injury compared to younger drivers possibly due to fragility. Older drivers do appear to pose an increased risk to their passengers. Collisions occurring at intersections, turning, and lane changes appear problematic to many older drivers.

\subsection{Aging and fitness-to-drive}

Part of the reason why older adult drivers may experience more collisions than younger drivers is due the natural course of aging. Cognitive and psychomotor declines, decreases in vision and/or hearing, and physical limitations could potentially lead to driving errors or increased risk of motor vehicle collision. The following sub-sections discuss the extant research on the association with aging and the ability for a driver to safely operate a motor vehicle (e.g. fitness-to-drive). 


\subsubsection{Cognition and fitness-to-drive}

Three studies have investigated the associations between cognitive declines, driving errors, and/or driving restrictions (McKnight and McKnight 1999; Anstey and Wood 2011; Rapoport, Naglie et al. 2013). All three studies revealed that decreased cognition is associated with more driving errors. Antsey et al evaluated the association age, cognitive function, and driving errors in a group of drivers $(n=266)$ aged 70-88 years. Participants performed several laboratory tests which evaluated their visual attention, processing speed, inhibition, reaction time, task switching along with an actual driving assessment. The authors determined that all error types increased with age of participants (Anstey and Wood 2011). Mcknight et al studied the association between cognitive function and unsafe driving incidents in 407 elderly drivers 62 year of age and older using several laboratory assessments and a driving test (McKnight and McKnight 1999). The authors found a significant association between participants with lower cognitive function and unsafe driving actions (McKnight and McKnight 1999). Rapoport et al examined the association between cognitive performance, driver perceptions, and self-reported driving restriction in a cohort of 928 drivers aged 70-94 years across several Canadian cities (Rapoport, Naglie et al. 2013). Cognition was evaluated laboratory assessment while driver perceptions were assessed using a scaled instrument. Those with lower cognitive functioning moderately reduced their driving frequency and avoidance of complex driving environments (Rapoport, Naglie et al. 2013).

Two studies have investigated how cognitive training can actually improve driver performance or prolong driving (Edwards, Delahunt et al. 2009; Ball, Edwards et al. 2010). Ball et al tested a randomized, cognitive intervention on 908 community dwelling seniors to see if increased cognitive training decreased the incidence of motor vehicle collisions (Ball, Edwards et al. 2010). Findings from the study showed that cognitive speed-of-processing and reasoning training resulted in a lower at-fault MVC rate in older drivers compared to controls (Ball, Edwards et al. 2010). Edwards et al also performed a cognitive function intervention $(n=276)$ to assess whether drivers with cognitive deficits who partook in the training would drive longer/later in life compared to controls (Edwards, Delahunt et al. 
2009). The results showed that drivers receiving the training were $40 \%$ less likely to cease driving compared to controls (Edwards, Delahunt et al. 2009).

\subsubsection{Psychomotor function and fitness-to-drive}

Three studies have investigated the relationship between psychomotor function and fitness-todrive (McKnight and McKnight 1999; Horswill, Pachana et al. 2009; Dawson, Uc et al. 2010). Dawson et al assessed the neuropsychological abilities in a laboratory setting and in an instrumented vehicle of older adults with no cognitive impairments $(n=111)$ compared to middle-aged adults ( $n=80)$ (Dawson, Uc et al. 2010). After adjusting for age, education, and sex, older drivers psychomotor functioning was significantly less than those of middle-aged adults (Dawson, Uc et al. 2010). A study conducted by Horswill et al also compared psychomotor function in young, middle and older adults and found that older adults had reduced response to perceived hazards compared to the other age groups (Horswill, Pachana et al. 2009). Mcknight et al conducted a psychomotor assessment and on the road driving assessment of 407 drivers over 62 years of age. The results showed that significant correlations were found between poor psychomotor functioning and unsafe driving errors (McKnight and McKnight 1999).

\subsubsection{Vision and fitness-to-drive}

The association between visual impairment and increased motor vehicle collision, decreased driving ability, and/or driving cessation has been extensively studied in the literature (Ivers, Mitchell et al. 1999; McGwin Jr, Chapman et al. 2000; Satariano, MacLeod et al. 2004; Ross, Anstey et al. 2009; Green, McGwin et al. 2013; Sandlin, McGwin et al. 2014). Green et al investigated the association between visual impairments and motor vehicle collision in a population based sample of 2,000 licensed drivers over 70 years of age in Alabama. Those with declines in contrast sensitivity were at a significantly higher risk of motor vehicle collision (Green, McGwin et al. 2013). A study by Ivers et al in examined the association between decreased visual acuity and motor vehicle collision in a cohort of individuals $(n=3,654)$ in Australia (Ivers, Mitchell et al. 1999). Compared to younger drivers, drivers over 80 years 
of age experienced significant more motor vehicle collisions if they experienced a decrease in visual acuity (Ivers, Mitchell et al. 1999). Mcgwin et al evaluated the association between visual function and driving difficulty in a group of drivers $(\mathrm{n}=384)$ (McGwin Jr, Chapman et al. 2000). Individuals with decreased visual acuity and contrast sensitivity were more likely to be involved in self-reported high-risk driving situations (McGwin Jr, Chapman et al. 2000). A study by Ross et al found that in an Australian cohort of adults over 65 years of age $(n=5,206)$, those with self-reported poor vision were more likely to cease driving compared to those with better self-reported vision (Ross, Anstey et al. 2009). A study by Sandlin et al examined the relationship between visual impairments and the amount that a person drives in a population-based sample of older adults in Alabama 70 years of age and older (Sandlin, McGwin et al. 2014). The results of the analysis showed that those with impaired contrast sensitivity reduced the number of trips and annual mileage compared to those with normal contrast sensitivity (Sandlin, McGwin et al. 2014). A study by Satariano et al also examined the relations between self-reported visual problems and driving cessation in a community-based study of adults $(n=1,840)$ over 55 years of age in Sonoma, California (Satariano, MacLeod et al. 2004). The results showed that drivers with more self-reported vision problems were more likely to reduce their driving trips/mileage (Satariano, MacLeod et al. 2004).

\subsubsection{Hearing and fitness-to-drive}

Three studies have investigated the relationship between hearing loss and fitness-to-drive (Ivers, Mitchell et al. 1999; Hickson, Wood et al. 2010; Green, McGwin et al. 2013). Green et al investigated the association between hearing impairment and motor vehicle collision in a population based sample of 2,000 licensed drivers over 70 years of age in Alabama (Green, McGwin et al. 2013). Drivers with hearing impairments were $52 \%$ more likely to be involved in a motor vehicle collision $(\mathrm{RR}=1.52,95 \%$ $\mathrm{CI}=1.01-2.30)$ (Green, McGwin et al. 2013). Hickson et al investigated the effects of hearing impairment on older adults driving ability and distractibility in a laboratory setting and an actual driving assessment (Hickson, Wood et al. 2010). It was found that older adult drivers with hearing impairments (both self-reported and clinically confirmed) had significantly poorer driving performance compared to 
those without impairments (Hickson, Wood et al. 2010). A study by Ivers et al in examined the association between hearing loss and motor vehicle collision in a cohort of individuals $(n=3,654)$ in Australia (Ivers, Mitchell et al. 1999). Compared to younger drivers, drivers over 80 years of age experienced significant more motor vehicle collisions if they experienced moderate hearing loss of loss of hearing in their right ears (Ivers, Mitchell et al. 1999). Therefore, declines in hearing may lead to increase in motor vehicle collision.

\subsubsection{Physical limitations and fitness-to-drive}

Two studies have investigated the relationship between physical limitations and fitness-to-drive (Reimer, D'Ambrosio et al. 2008; Antin, Lockhart et al. 2012). A study by Antin compared 49 drivers and non-drivers 65 years of age and older on various physical and mental assessments (Antin, Lockhart et al. 2012). Results showed that non-drivers had more physical and cognitive limitations than those who continued to drive (Antin, Lockhart et al. 2012). A study by Reimer et al investigated the effects of flexibility on safe vehicle operation in younger and older drivers (Reimer, D'Ambrosio et al. 2008). Results showed that while older drivers were less flexible than younger drivers, many had developed compensatory measures (i.e. bracing) to overcome flexibility limitations to prolong their driving (Reimer, D'Ambrosio et al. 2008).

\subsection{Medical conditions associated with motor vehicle collision risk}

While declines in cognition, psychomotor function, vision, hearing, and physical limitations associated with the normal aging process may pose a risk to drivers' safety, numerous medical conditions have been linked to increased motor vehicle collision risk and/or decreased driving ability. Alzheimer's/dementias (Fitten, Perryman et al. 1995; Tuokko, Tallman et al. 1995; Cox, Quillian et al. 1998; Duchek, Carr et al. 2003; Frittelli, Borghetti et al. 2009; Eby, Silverstein et al. 2012), anxiety/depression (Sagberg 2006; Wingen, Ramaekers et al. 2006), arrhythmias (Stewart 1993; Gresset and Meyer 1994), cataracts (Owsley, Stalvey et al. 1999; Owsley, McGwin Jr et al. 2002), glaucoma 
(Haymes, Leblanc et al. 2007), chronic obstructive pulmonary disease (Karakontaki, Gennimata et al. 2013), cardiovascular diseases (Koepsell, Wolf et al. 1994; Guibert, Potvin et al. 1998; Lundqvist, Gerdle et al. 2000; Sagberg 2006; Hours, Fort et al. 2008), diabetes (Hansotia and Broste 1991; Koepsell, Wolf et al. 1994; McGwin, Sims et al. 1999; Sagberg 2006; Redelmeier, Kenshole et al. 2009; Signorovitch, Macaulay et al. 2013), epilepsy (Hansotia and Broste 1991; Beaussart, Beaussart-Defaye et al. 1997), Parkinson's disease (Lings and Dupont 1992; Classen, Brumback et al. 2014), and sleep apnea (George, Boudreau et al. 1996; Mulgrew, Nasvadi et al. 2008; Gieteling, Bakker et al. 2012) are all associated with increased risk of motor vehicle collision and/or decreased driving ability.

The associations between Alzheimer's disease/dementias (AD) and motor vehicle collision risk and/or affected driving ability have been studied using driving simulators, instrumented vehicles, or collision rates. Cox et al performed a matched case control study of 29 patients with Alzheimer's disease (AD) using a driving simulator to understand how driving is related to mental status (Cox, Quillian et al. 1998). Those with AD performed much worse than controls (Cox, Quillian et al. 1998). Frittelli et al also assessed the driving performance of $20 \mathrm{AD}$ patients, 20 patients with mild cognitive impairment, and 20 healthy controls using a driving simulator. Those with AD performed significantly worse than those with mild cognitive impairments and controls (Frittelli, Borghetti et al. 2009). Duckeck et al assessed the driving performance of $\mathrm{AD}$ patients with mild/early symptoms to those of healthy controls in a longitudinal study (Duchek, Carr et al. 2003). Those with early AD were significantly more likely to experience decreased driving performance compared to controls. These findings suggest even at early disease onset, those with AD may have compromised driving ability (Duchek, Carr et al. 2003). Eby et al assessed the driving performance of early stage dementia patients $(n=17)$ compared to healthy controls $(n=26)$ using instrumented vehicles (Eby, Silverstein et al. 2012). While driving performance did not differ vastly between groups, those with dementia did not drive as far and were more likely to become lost than controls (Eby, Silverstein et al. 2012). Tuokko et al retrospectively assessed the driving records of patients receiving treatment for dementia $(n=249)$ and compared these to a matched cohort of patients 
at the same clinic without diagnosed dementia (Tuokko, Tallman et al. 1995). The authors found that those with dementia had a collision rate 2.5 times greater than the control group (Tuokko, Tallman et al. 1995). Consequently, there is evidence that $\mathrm{AD}$ affects driving ability, but it may be dependent on the severity of cognitive impairment more than diagnosis (Fitten, Perryman et al. 1995).

Two studies have assessed the relationship between anxiety/depression, motor vehicle collision, and/or affected driving ability (Sagberg 2006; Wingen, Ramaekers et al. 2006). Sagberg et al administered a self-reported questionnaire to older drivers $(n=4,448)$ to assess which medical conditions and symptoms may be associated with motor vehicle collision. Patients who reported being anxious or depressed had significantly higher odds of motor vehicle collision (Sagberg 2006). Wingen et al assessed the driving performance of clinically depressed patients compared to healthy controls. Those with depression had poorer driving performance and were more likely to weave within driving lanes and have slower reaction times compared to controls (Wingen, Ramaekers et al. 2006).

Two studies have investigated the relationship between arrhythmias and motor vehicle collision or affected driving ability (Stewart 1993; Gresset and Meyer 1994). Gresset et al conducted a matched case control study of men aged 70 years or older residing in Quebec who had been involved in motor vehicle collisions (Gresset and Meyer 1994). The purpose was to assess which self-reported medical conditions were associated with higher odds of collision. Those with heart arrhythmias were 1.63 times more likely to be involved in collisions (OR=1.63, CI: 1.00-2.65) (Gresset and Meyer 1994). Stewart et al assessed the risk of motor vehicle collision by self-reported medical conditions in a cohort of Florida residents (Stewart 1993). Heart arrhythmias were a statistically significant predictor of motor vehicle collision in this population (Stewart 1993).

Three studies have investigated the association between motor vehicle collision and optometric diseases such as cataracts (Owsley, Stalvey et al. 1999; Owsley, McGwin Jr et al. 2002) and glaucoma (Haymes, Leblanc et al. 2007). Owsley et al compared the collision data of those with and without 
cataracts in a population of older drivers in Alabama (Owsley, Stalvey et al. 1999). Those with cataracts were 2.5 times more likely to be involved in an at-fault motor vehicle collision compared to those without the disease (Owsley, Stalvey et al. 1999). Additional studies by the same research group showed that cataract surgery can actually reduce collision risk (Owsley, McGwin Jr et al. 2002). Haymes et al conducted a matched case control study to investigate the odds of motor vehicle collision in a group of patients with glaucoma compared to a control group (Haymes, Leblanc et al. 2007). After adjusting for annual miles driven, glaucoma patients were involved in motor vehicle collisions 6 times more than controls (Haymes, Leblanc et al. 2007).

One study has investigated the effects of chronic obstructive pulmonary disease (COPD) on skills related to driving performance. COPD patients $(n=35)$ and healthy controls $(n=10)$ underwent a battery of psychomotor testing related to driving skills. COPD patients performed significantly worse than controls on all measurements (Karakontaki, Gennimata et al. 2013).

Several studies have assessed the relationship between cardiovascular ailments (i.e. heart attack, stroke, hypertension, coronary artery disease) and motor vehicle collision (Koepsell, Wolf et al. 1994; Guibert, Potvin et al. 1998; Lundqvist, Gerdle et al. 2000; Sagberg 2006; Hours, Fort et al. 2008). The findings are often mixed. Guibert et al conducted a population-based case control study in Quebec, Canada to assess whether male drivers with self-reported cardiovascular disease were more likely to be involved in a motor vehicle collision. After adjusting for annual miles driven, those with cardiovascular disease experienced fewer motor vehicle collisions (Guibert, Potvin et al. 1998). Sagberg et al administered a self-reported questionnaire to older drivers $(n=4,448)$ to assess which medical conditions and symptoms may be associated with motor vehicle collision. Patients who reported having a previous heart attack had significantly higher odds (OR: 1.77) of motor vehicle collision (Sagberg 2006).

Lundqvist et al conducted a case control study to assess the effects of stroke on driving performance using psychomotor testing, a driving simulator, and actual driving assessment. Stoke patients performed worse than controls in all testing environments (Lundqvist, Gerdle et al. 2000). Koepsell et al conducted a case 
control study to assess which medical conditions may be associated with motor vehicle collision in a group of drivers over 65 years of age living in Washington State. Those diagnosed with coronary heart disease were $40 \%$ more likely to be involved in a motor vehicle collision (OR: 1.4; 95\% CI 1.0, 2.2) (Koepsell, Wolf et al. 1994). Hours et al also conducted a nested case control study to assess which medical conditions were associated with motor vehicle collision in a cohort of individuals living in Rhone, France. Those with hypertension were at a significantly increased risk of motor vehicle collision (OR: 3.82, 95\% CI 1.42, 10.24) (Hours, Fort et al. 2008).

Several studies have investigated the relationship between type- 1 or type- 2 diabetes and the odds/risk of motor vehicle collision (Hansotia and Broste 1991; Koepsell, Wolf et al. 1994; McGwin, Sims et al. 1999; Sagberg 2006; Redelmeier, Kenshole et al. 2009; Signorovitch, Macaulay et al. 2013). The findings of these studies are often mixed. Hansotia et al conducted a population-based retrospective cohort study $(n=30,420)$ of adults with and without diabetes in Marshfield, Wisconsin. Those with diabetes experienced $32 \%$ more collisions and $14 \%$ more moving violations compared to those without the condition (Hansotia and Broste 1991). Mcgwin et al conducted a population-based case control study of drivers over 65 years of age in Alabama to assess if those with diabetes experienced more motor vehicle collisions. Those with diabetes were not at a higher risk of collisions or at-fault collisions compared to controls (McGwin, Sims et al. 1999). Koepsell et al conducted a case control study to assess which medical conditions may be associated with motor vehicle collision in a group of drivers over 65 years of age living in Washington State. Those diagnosed with diabetes were 2.6 times more likely to be involved in a motor vehicle collision (OR: 2.6; 95 \% CI 1.4, 4.7) (Koepsell, Wolf et al. 1994). Redelmeier et al studied that association between glycosylated hemoglobin, diabetes and motor vehicle collision via a population-based case control study. Findings suggested that even with tight glycemic control (i.e. improved glycosylated hemoglobin levels), the odds of motor vehicle collisions were still higher than controls (Redelmeier, Kenshole et al. 2009). Sagberg et al administered a self-reported questionnaire to older drivers $(n=4,448)$ to assess which medical conditions and symptoms may be 
associated with motor vehicle collision. Patients who reported non-medicated diabetes had 3.08 higher odds of motor vehicle collision (Sagberg 2006). Signorovitch et al assessed the association between motor vehicle collision and those who had type-2 diabetes that were not treated with insulin via a retrospective cohort study using a medical claims database $(n=5582)$. Those admitted for hypoglycemic attacks were also at a higher risk of being admitted for motor vehicle collisions (HR 1.82; 95\% CI 1.18, 2.80) (Signorovitch, Macaulay et al. 2013).

Two studies have investigated the association between epilepsy and motor vehicle collisions (Hansotia and Broste 1991; Beaussart, Beaussart-Defaye et al. 1997). Beaussart et al conducted a longitudinal study of epileptic patients to assess the relationship between motor vehicle collision and epileptic seizures while driving (Beaussart, Beaussart-Defaye et al. 1997). Results showed that the incidence of seizures was very low and severities of collisions were minor. Hansotia et al conducted a population-based retrospective cohort study $(n=30,420)$ of adults with and without epilepsy in Marshfield, Wisconsin. Those with epilepsy experienced 33\% more collisions compared to those without the condition (Hansotia and Broste 1991).

Two studies have investigated how driving ability is affected by Parkinson's disease (PD) (Lings and Dupont 1992; Classen, Brumback et al. 2014). Classen et al compared the driving ability of PD patients $(n=101)$ to healthy controls $(n=138)$ via an actual driving assessment. PD patients made significantly more driving errors overall compared to controls (Classen, Brumback et al. 2014). Lings et al used a driving simulator to assess the driving ability of PD patients $(\mathrm{n}=28)$ compared to healthy controls $(\mathrm{n}=109)$. Those with PD had more driving errors, such as failure to react, reduced strength, and increased reaction times, compared to controls (Lings and Dupont 1992).

Three studies have investigated how sleep apnea affects motor vehicle collision risk and/or driving ability (George, Boudreau et al. 1996; Mulgrew, Nasvadi et al. 2008; Gieteling, Bakker et al. 2012). Mulgrew et al investigated the association between obstructive sleep apnea and motor vehicle 
collision via a case-control study $(n=783)$. Those with sleep apnea were at a significantly increased risk of collision (Mulgrew, Nasvadi et al. 2008). George et al investigated the driving ability of male patients with sleep apnea $(n=21)$ matched by sex and age to those without the condition $(n=21)$ using a driving simulator. Patients with sleep apnea performed worse on all tests compared to controls (George, Boudreau et al. 1996). Geiteling et al also investigated the driving ability of sleep apnea patients $(\mathrm{n}=18)$ compared to a control group $(\mathrm{n}=18)$ and a group of patients with periodic limb movement disorder $(\mathrm{n}=18)$ via a driving simulation experiment. Those with sleep apnea and periodic limb movement disorder performed slightly worse than controls over the course of the experiment, particularly regarding vigilance (Gieteling, Bakker et al. 2012).

\subsection{Licit drugs and motor vehicle collision risk}

While the natural aging process and existing medical conditions can influence motor vehicle collision risk and/or driving ability, so can prescription or over-the-counter medication usage. It is wellestablished in the literature that medication usage increases with age. While the number of prescription and over-the-counter medications (i.e. licit drugs) taken each day can vary between individuals, it is estimated that approximately $60 \%$ of all adults 65 years of age and older consume one prescription medication daily (Fulton and Allen 2005), while only $20 \%$ use three or more prescriptions each day (Jorgensen, Johansson et al. 2001). Current research also suggests that adults 65 years of age an older consume approximately $30 \%$ of annual written prescriptions (Bushardt, Massey et al. 2008). This age group is also the largest consumers of over-the-counter medications based on retail drug sales (Fulton and Allen 2005). Unfortunately, both prescription and over-the-counter medication may produce side effects that interfere with safe driving. Numerous medications have been associated with motor vehicle collision risk and/or decreased driving ability. The following sections review the current research pertaining to this issue.

\subsubsection{The effects of licit drugs on motor vehicle collision risk}


Most of the research pertaining to the relationship between licit drug use and motor vehicle collision risk has grouped and analyzed these drugs as broad categories. The majority of drugs studied have included psychotropic drugs, which include antidepressants, antipsychotics, benzodiazepines and zdrugs (i.e. sleep medications). Narcotic analgesics have also been studied extensively, in addition to antidiabetic drugs.

At least twelve studies have investigated the risk posed by antidepressant medication (Ray, Fought et al. 1992; Leveille, Buchner et al. 1994; Bramness, Skuytveit et al. 2008; Hours, Fort et al. 2008; Rapoport, Herrmann et al. 2008; Coupland, Dhiman et al. 2011; Meuleners, Duke et al. 2011; Rapoport, Zagorski et al. 2011; Ravera, van Rein et al. 2011; Orriols, Queinec et al. 2012; Chang, Wu et al. 2013; Orriols, Wilchesky et al. 2013). The results of these studies are often mixed. Bramness et al found that sedating (Incidence ratio, IR 1.4; 95\% Cl 1.2, 1.6) and non-sedating (IR 1.6; 95\% CI 1.5, 1.7) antidepressants were associated with increased collision risk in a retrospective cohort of drivers aged 1869 years in Norway (Bramness, Skuytveit et al. 2008). In a matched case-control study in Taiwan using claims data, Chang et al found that antidepressant use one week or one month was significantly associated with motor vehicle collisions (Chang, Wu et al. 2013). In a large cohort study ( $n=60,746)$ of clinically depressed adults over 65 years of age conducted in England using claims data, Coupland et al found that only certain individual antidepressants were associated with collision risk (Coupland, Dhiman et al. 2011). In a case-control study conducted in France, Hours et al found that antidepressant use was significantly associated with at-fault motor vehicle collisions (OR 3.6; 95\% CI 1.3, 10.0) (Hours, Fort et al. 2008). In a case control study conducted in Seattle, WA of drivers over 65 years of age, Leveille et al found that tricyclic antidepressant use was associated with a 2.3 increased risk of collision (Leveille, Buchner et al. 1994). In a population-based case-crossover study conducted in Australia, Meuleners et al found that men who were prescribed an antidepressant were at an increased risk of collision while females were not (Meuleners, Duke et al. 2011). In a population-based case-crossover study conducted in France, Orriols et al found that when antidepressant medications were initiated or changed, those who consumed 
these medications were at a $49 \%$ and $32 \%$, respectively, higher risk of motor vehicle collision (Orriols, Queinec et al. 2012). In a case-crossover study conducted in Quebec, Canada among drivers over 65 years of age $(n=109,406)$, drivers who consumed antidepressants one month before their crash were at a higher risk of collision compared to antidepressant use that occurred 4-8 months before the collision (Orriols, Wilchesky et al. 2013). In a case-crossover study conducted in Ontario, Canada among adults over 65 years of age diagnosed with dementia, Rapoport et al found that second generation antidepressant use (i.e. newer antidepressant medication) was associated with a higher collision risk than first generation antidepressants (i.e. older antidepressant medications) (Rapoport, Herrmann et al. 2008). In a retrospective cohort study conducted in Ontario, Canada among adult driver over 65 years of age, second generation antidepressants were associated with a modest increase (i.e. 10\%) in motor vehicle collision (OR 1.10; 95\% CI 1.07, 1.13) (Rapoport, Zagorski et al. 2011). In a case control study conducted in the Netherlands, Ravera et al found that younger female drivers who were prescribed second generation antidepressants were at an increased risk of collision (Ravera, van Rein et al. 2011). In a retrospective cohort study of Medicaid enrollees over 65 years of age in Tennessee, Ray et al found that tricyclic antidepressant use was associated with a two-fold increase of motor vehicle collision (relative risk, RR 2.2; 95\% CI 1.3, 3.5) (Ray, Fought et al. 1992).

At least eight studies have investigated the association between motor vehicle collision and benzodiazepine use; all studies show that benzodiazepine use is associated with motor vehicle collision (Ray, Fought et al. 1992; Barbone, McMahon et al. 1998; Neutel 1998; England, Skurtveit et al. 2007; Rapoport, Herrmann et al. 2008; Meuleners, Duke et al. 2011; Orriols, Philip et al. 2011; Chang, Wu et al. 2013). In a cohort study conducted in Norway of 18-70 year old drivers ( $n=3.1$ million), those receiving a prescription for benzodiazepine tranquilizers or hypnotics were at a significantly increased risk of motor vehicle collision (England, Skurtveit et al. 2007). Barbone et al conducted a case-crossover study of driver over 18 years of age in Scotland and found that benzodiazepine use was associated with a $62 \%$ increased risk of collision (OR 1.62; 95\% CI 1.24, 2.12) (Barbone, McMahon et al. 1998). In a 
matched case-control study in Taiwan using claims data, Chang et al found that benzodiazepine use one week or one month was significantly associated with motor vehicle collision (Chang, Wu et al. 2013). In a population-based case-crossover study conducted in Australia of drivers 60 years of age and older, Meuleners et al found that both men and women who were prescribed a benzodiazepine were at an increased risk of collision (Meuleners, Duke et al. 2011). In a cohort study conducted in Saskatchewan, Canada $(n=225,796)$ which compared driver over 60 years of age to those under 60 , Neutel et al found that regardless of age, benzodiazepine use increased the risk of motor vehicle collision $(\leq 60$ years: OR 3.2; 95\% CI 1.3, 3.8; >60 years: OR 2.8; 95\% CI 1.0, 8.4) (Neutel 1998). In a population-based retrospective cohort study conducted in France, Orriols et al found that benzodiazepine hypnotic use was significantly associated with a 39\% higher risk of motor vehicle collision (Orriols, Philip et al. 2011). In a case-crossover study conducted in Ontario, Canada among adults over 65 years of age diagnosed with dementia, Rapoport et al found that benzodiazepine use was associated with a higher collision risk (Rapoport, Herrmann et al. 2008). In a retrospective cohort study of Medicaid enrollees over 65 years of age in Tennessee, Ray et al found that benzodiazepine use was associated with a $50 \%$ increase of motor vehicle collision (Ray, Fought et al. 1992).

At least three studies have investigated antihyperglycemic drugs and motor vehicle collision risk; the findings are often mixed (Hemmelgarn, Lévesque et al. 2006; Lonnen, Powell et al. 2008; Skurtveit, Strøm et al. 2009). In a nested case-control study conducted in Quebec, Canada, those taking insulin were at an increased risk of collision while those taking sulfonylurea or metformin were not (Hemmelgarn, Lévesque et al. 2006). In a retrospective cohort study conducted in England, Lonnen et al found that those taking insulin were not at an increased risk of motor vehicle collision (Lonnen, Powell et al. 2008). In a cohort study conducted in Norway of 18-70 year old drivers ( $\mathrm{n}=3.1$ million), those receiving a prescription for insulin or glucose-lowering agents were at a $40 \%$ and $20 \%$, respective, increased risk of motor vehicle collision (Skurtveit, Strøm et al. 2009). 
At least three studies have investigated the relationship between hypnotics (i.e. z-drugs) and motor vehicle collision risk; all three studies have determined an increased risk of motor vehicle collision with their use (Gustavsen, Bramness et al. 2008; Yang, Lai et al. 2011; Chang, Wu et al. 2013). In a matched case-control study in Taiwan using claims data, Chang et al found that the use of z-drugs for one week or one month was significantly associated with motor vehicle collision (Chang, Wu et al. 2013). In a cohort study conducted in Norway of 18-70 year old drivers ( $\mathrm{n}=3.1$ million), those receiving a prescription for z-drugs were at an increased risk of motor vehicle collision (OR 2.3; 95\% CI 2.0, 2.7) (Gustavsen, Bramness et al. 2008). In randomly selected cohort of 1 million people established using medical claims data in Taiwan, Yang et al found that taking one dose of a z-drug was associated with a 74\% increased risk of collision (Yang, Lai et al. 2011).

At least eight studies have investigated the relationship between narcotic use and motor vehicle collision risk; most show that narcotic use is associated with motor vehicle collision risk (Leveille, Buchner et al. 1994; England, Skurtveit et al. 2007; Bachs, Engeland et al. 2009; Majdzadeh, Feiz-Zadeh et al. 2009; Meuleners, Duke et al. 2011; Bramness, Skurtveit et al. 2012; Corsenac, Lagarde et al. 2012; Gomes, Redelmeier et al. 2013). In a cohort study conducted in Norway of 18-70 year old drivers ( $\mathrm{n}=3.1$ million), those receiving a prescription for opioids were at a significantly increased risk of motor vehicle collision (England, Skurtveit et al. 2007); this study was repeated by Bachs et al, but for codeine or Tramadol specifically, and these two drugs were found to be associated with increased collision risk (Bachs, Engeland et al. 2009). Bramness et al repeated the Bachs et al study for methadone and found that only methadone use in men were associated with increased collision risk (IR 2.4; 95\% CI 1.5, 3.6) (Bramness, Skurtveit et al. 2012). Corsneac et al performed a population based case control study in France $(n=72,865)$ and found that buprenorphine or methadone use were both associated with an increased risk of collision (OR 2.0; 95\% CI 1.4, 2.9) (Corsenac, Lagarde et al. 2012). Gomes et al conducted a population-based nested case control study of patients aged 18 to 64 years who received at least one publicly funded prescription for an opioid and determined that the higher the opioid dose, the 
higher risk of motor vehicle collision (Gomes, Redelmeier et al. 2013). In a case control study conducted in Seattle, WA of drivers over 65 years of age, Leveille et al found opioid use was associated with a $88 \%$ increased risk of collision (Leveille, Buchner et al. 1994). In a case-crossover study of drivers injured in motor vehicle collision who required medical treatment in Kerman, Iran, those using opium six hours before the collision were at an increased risk of collision (Majdzadeh, Feiz-Zadeh et al. 2009). In a population-based case-crossover study conducted in Australia of drivers 60 years of age and older, Meuleners et al found that those taking narcotic analgesics were at an increased risk of collision (OR 1.5; 95\% CI 1.0-2.3) (Meuleners, Duke et al. 2011).

\subsubsection{The effects of licit drugs on actual driving ability}

While numerous licit drugs have been associated with increased motor vehicle collision risk, many others have been evaluated in experimental studies to determine if the drugs affect actual driving ability. Most of the research involving drugs and driving ability have centered on antidepressants, antihistamines, benzodiazepines, hypnotics (i.e. z medications), and opioids. The results of the experimental studies need to be interpreted with caution as the sample sizes are often small and limited to younger or healthier participants; hence, the findings may not be generalizable to older populations with complex medical conditions or multiple medication use.

At least ten experimental, placebo controlled, cross-over studies have investigated specific antidepressant medications and their effects on driving ability; the findings from these studies are often dependent on the drug and/or population being tested (Ramaekers, Swijgman et al. 1992; Ramaekers, Muntjewerff et al. 1995; Ramaekers, Ansseau et al. 1997; O'Hanlon, Robbe et al. 1998; Ramaekers, Muntjewereff et al. 1998; Ridout and Hindmarch 2001; Ridout, Meadows et al. 2003; Wingen, Bothmer et al. 2005; Wingen, Ramaekers et al. 2006; Theunissen, Street et al. 2013). O'hanlon et al found that Venlafaxine did not impair driving performance among 37 healthy volunteers (O'Hanlon, Robbe et al. 1998). Ramekers et al found that the driving performance of 41 clinically depressed patients was actually 
improved with use of Moclobemide or Fluoxetine (Ramaekers, Ansseau et al. 1997). Raemakers et al also found that among 18 healthy volunteers, nocturnal doses of Mirtazapine or Mianserin did not affect driving performance as much as when given during the daytime (Ramaekers, Muntjewereff et al. 1998). Raemakers et al found that driving performance of 18 healthy volunteers was not affected by Dothiepin or Fluoxetine (Ramaekers, Muntjewerff et al. 1995). Raemakers et al found that among 17 healthy volunteers, Mianserin affected driving performance while Moclobemide had not effects (Ramaekers, Swijgman et al. 1992). In a study of 12 healthy volunteers conducted by Ridout et al, Tianeptine did not affect performance any different than placebo, but Mianserin did impair driving performance (Ridout and Hindmarch 2001). In a study of 16 healthy volunteers conducted by Ridout et al, Paroxetine did not affect performance any different than placebo, but Mirtazapine did impair driving performance (Ridout, Meadows et al. 2003). In a study of 24 healthy subjects, Theunissen et al determined that Mirtazapine effects driving performance while Vortioxetine did not compared to placebo (Theunissen, Street et al. 2013). Wingen et al performed a study using 18 healthy subjects and found Mirtazapine affected driving performance while Escitalopram did not compared to placebo (Wingen, Bothmer et al. 2005). Wingen et al tested the driving performance of clinically depressed patients compared to healthy participants; depressed patients taking second generation antidepressants performed worse on all measure compared to controls (Wingen, Ramaekers et al. 2006).

Three, experimental, placebo controlled, cross-over studies have assessed the effects of narcotics on driving ability (Byas-Smith, Chapman et al. 2005; Verster, Veldhuijzen et al. 2006; Amato, Marie et al. 2013). Amato et al assessed the effects of increasing doses of codeine/paracetamol on sixteen healthy volunteers and found no performance deficits compared to placebo (Amato, Marie et al. 2013). ByasSmith et al compared the driving ability of chronic pain patients taking opioid analgesics to healthy volunteers and found no significant differences in performance between the groups (Byas-Smith, Chapman et al. 2005). Verster et al compared the driving ability of 18 patients taking Bromfenac, a nonsteroidal anti-inflammatory, and the opioid oxycodone/paracetamol and found no significant impairment 
between oxycodone/paracetamol and placebo in a standardized road test (Verster, Veldhuijzen et al. 2006).

Five, experimental, placebo controlled, cross-over studies have investigated the effects of z-drugs (i.e. hypnotics) on driving performance; results show that some drugs are more impairing than others (Vermeeren, Danjou et al. 1998; Vermeeren, Riedel et al. 2002; Verster, Volkerts et al. 2002; Leufkens, Lund et al. 2009; Mets, Vries et al. 2011). Leufkens et al conducted a study with 28 healthy volunteers to assess the effects of Gaboxadol, Zopiclone, and Zolpidem on driving performance and found that all three drugs impaired performance (Leufkens, Lund et al. 2009). Mets et al conducted a study with 30 healthy volunteers to evaluate the effects of Ramelteon and Zopiclone on driving performance and found both to be impairing (Mets, Vries et al. 2011). Vermeeren et al conducted two studies with 28 and 30 healthy volunteers to evaluate the effects of Zaleplon and Zopiclone on driving performance and found that Zopiclone was impairing but Zaleplon is probably safe if taken at least five hours before driving in both studies (Vermeeren, Danjou et al. 1998; Vermeeren, Riedel et al. 2002). Verster et al conducted an experiment with 30 healthy volunteers to assess the effects of Zaleplon and Zolpidem on driving performance and found that Zolpidem impairs performance while Zaleplon is relatively safe if taken several hours before driving (Verster, Volkerts et al. 2002).

Seventeen, experimental, placebo controlled, cross-over studies have been conducted to assess the effects that antihistamines have on actual driving ability using instrumented vehicles; some antihistamines appear driver impairing while others are not (Hindmarch 1976; Ramaekers, Uiterwijk et al. 1992;

Volkerts, Van et al. 1992; Ramaeker and O'Hanlon 1994; Vuurman, Uiterwijk et al. 1994; Vermeeren and O'Hanlon 1998; Vermeeren, Ramaekers et al. 1998; Vermeeren, Ramaekers et al. 2002; Potter, Schepers et al. 2003; Ridout, Shamsi et al. 2003; Verster, Weert et al. 2003; Theunissen, Vermeeren et al. 2004; Vuurman, Rikken et al. 2004; Tashiro, Horikawa et al. 2005; Theunissen, Vermeeren et al. 2006; Vuurman, Eef et al. 2007; Conen, Theunissen et al. 2011). Conen et al conducted a study of Bilastine and Hydroxyzine in a group of 22 healthy participants and determined that Hydroxyzine affected driving 
while Bilastine did not (Conen, Theunissen et al. 2011). Hindmarch et al conducted a study of Clemastine in a group of 21 healthy volunteers and found that Clemastine did not affect driving ability (Hindmarch 1976). Potter et al evaluated Fexofenadine in 255 healthy volunteers and determined that driving errors were not increased compared to placebo (Potter, Schepers et al. 2003). Ramaeker et al evaluated Acrivastine, Terfenadine, and Diphenhydramine in 18 healthy volunteers and found that high doses of Acrivastine and regular doses of Diphenhydramine affected driving performance while Terfenadine had no significant effects on driving compared to placebo (Ramaeker and O'Hanlon 1994). Ramaeker et al tested the effects of Cetirizine and Loratadine in 16 healthy volunteers and found that Cetirizine caused mild driving impairment while Loratadine did not (Ramaekers, Uiterwijk et al. 1992). Ridout et al evaluated Fexofenadine and Hydroxyzine in 18 healthy volunteers and found that Fexofenadine did not cause impairment while Hydroxyzine did cause impairment compared to placebo (Ridout, Shamsi et al. 2003). Tashiro et al also evaluated Fexofenadine and Hydroxyzine in healthy volunteers and found Fexofenadine did not impair performance while Hydroxyzine increased reaction time compared to placebo (Tashiro, Horikawa et al. 2005). Theunissen et al conducted two studies of Mequitazine, Cetirizine and Dexchlorpheniramine in 16 and 18 healthy volunteers and found that only Cetirizine did not impair driving performance in either study (Theunissen, Vermeeren et al. 2004; Theunissen, Vermeeren et al. 2006). Vermeeren et al evaluated the effects of Fexofenadine and Clemastine in 24 healthy volunteers and found that Fexofenadine did not impair performance compared to placebo (Vermeeren and O'Hanlon 1998). Vermeeren et al evaluated the effects of Emedastine and Cetirizine in a group of 19 healthy volunteers and found that both drugs are sedating compared to placebo (Vermeeren, Ramaekers et al. 2002). Vermeeren et al tested a combination of Chlorpheniramine and Terfenadine in a group of 24 healthy volunteers and found no significant differences in driving performance compared to placebo (Vermeeren, Ramaekers et al. 1998). Verster et al studied both Levocitirizine and Diphenhydramine in a group of 48 healthy volunteers and determined that Levocitirizine does not affect driving performance while Diphenhydamine does compared to placebo (Verster, Weert et al. 2003). Volkerts et al tested Cetirizine and Triprolidine in 27 healthy male 
volunteers and found Ceterizine did not affect driving while Triprolidine significantly impaired performance (Volkerts, Van et al. 1992). Vuurman et al studied Rupatadine and Hydroxyzine in a group of 20 healthy subjects and found that Rupatadine, but not Hydroxyzine, does not impair driving performance (Vuurman, Eef et al. 2007). Vuurman et al evaluated the effects of Desloratadine and Diphenhydramine in 18 healthy volunteers and found that therapeutic doses of Desloratadine do not affect driving performance while Diphenydramine caused participants to swerve with their driving lanes (Vuurman, Rikken et al. 2004). Vuurman et al studied the effects of Mizolastine and Clemastine in 24 healthy volunteers and found that Mizolastine does not appear to affect driving ability (Vuurman, Uiterwijk et al. 1994).

Benzodiazepines are the most studied drug class when it comes to evaluating their effects on driving performance. At least 22 randomized, placebo-controlled, experimental studies have been conducted to date in healthy, depressed, and/or anxious volunteers; the results of these studies show that some benzodiazepines are associated with decreased driving ability while others are not (Hindmarch, Hanks et al. 1977; Hindmarch and Gudgeon 1980; De Gier, Hart et al. 1981; O'Hanlon, Haak et al. 1982; Hindmarch and Subhan 1983; Laurell and Tornros 1986; Schmidt, Brendemühl et al. 1986; Stevenson, Pathria et al. 1986; Riedel, Quasten et al. 1988; Brookhuis, Volkerts et al. 1990; Willumeit, Ott et al. 1991; Van Laar, Volkerts et al. 1992; Volkerts, Laar et al. 1992; Van Laar, Van Willigenburg et al. 1993; Mercier-Guyon, Lejay et al. 1999; Van Laar, Volkerts et al. 2001; Verster, Volkerts et al. 2002; Hindmarch, Trick et al. 2005; Leufkens, Vermeeren et al. 2007; Leufkens and Vermeeren 2009; Daurat, Sagaspe et al. 2013; Moták, Bayssac et al. 2014). The following benzodiazepines have been associated with decreased driving performance: Lormetazepam (Brookhuis, Volkerts et al. 1990), Flurazepam (Brookhuis, Volkerts et al. 1990), Lorazepam (Hindmarch and Gudgeon 1980; Mercier-Guyon, Lejay et al. 1999; Van Laar, Volkerts et al. 2001; Daurat, Sagaspe et al. 2013; Moták, Bayssac et al. 2014), Diazepam (De Gier, Hart et al. 1981; O'Hanlon, Haak et al. 1982; Stevenson, Pathria et al. 1986; Van Laar, Volkerts et al. 1992), Alprazolam (Verster, Volkerts et al. 2002; Hindmarch, Trick et al. 2005; 
Leufkens, Vermeeren et al. 2007), Nitrazepam (Laurell and Tornros 1986), Oxazepam (Volkerts, Laar et al. 1992; Van Laar, Van Willigenburg et al. 1993), and Flunitrazepam (Schmidt, Brendemühl et al. 1986). The following benzodiazepines have been shown to not significantly affect driving performance compared to placebo: Clobazam (Hindmarch, Hanks et al. 1977; Hindmarch and Gudgeon 1980), Midazolam (Hindmarch and Subhan 1983; Riedel, Quasten et al. 1988), Triazolam (Laurell and Tornros 1986; Riedel, Quasten et al. 1988), and Temazepam (Schmidt, Brendemühl et al. 1986; Riedel, Quasten et al. 1988; Leufkens and Vermeeren 2009). Lormetazepam has shown to be relatively safe as long as patients sleep a full night prior to driving (Willumeit, Ott et al. 1991; Volkerts, Laar et al. 1992; Van Laar, Van Willigenburg et al. 1993).

\subsection{Illicit drugs and motor vehicle collision risk}

While licit drugs, such as prescription and over-the-counter medication, can influence driving ability or collision risk, so can illicit drugs. Illicit drugs can be defined as drugs with no real medical benefit that are taken for recreational purposes. Current research suggests that illicit drug use, particularly opiates and stimulants, are increasing among older adult populations across the U.S. and Europe (Beynon 2009). Despite this fact, to the author's knowledge, no studies to date have investigated illicit drug use among older adult drivers. Only one study conducted by Vanderbilt University during the 1990's showed that illegal drug use was virtually non-existent among drivers over 65 years of age who were admitted to their level-1 trauma center after motor vehicle collision (Higgins, Wright et al. 1996). While the research in this area is extremely limited, it is possible that those older adults who use illicit drugs do not drive or do not drive regularly. Despite the lack of research in older adult populations, the following section highlights the current research surrounding the effects on illicit substances on collision risk and/or affected driving ability.

\subsubsection{The effects of individual illicit drugs on motor vehicle collision risk}


The associations between illegal drug use and motor vehicle collision have been studied extensively for marijuana, but also to a lesser extent for cocaine and heroin. Other studies have looked at broad groups of illegal drugs, such as opiates, stimulants, and depressants.

The association between marijuana and motor vehicle collision has been studied extensively in different populations among different countries using different study designs; of the 11 studies that have investigated this drug, all have found statistically significant increases of motor vehicle collision when drivers use this substance (Ferguson and Horwood 2001; Laumon, Gadegbeku et al. 2005; Mura, Brunet et al. 2006; Bedard, Dubois et al. 2007; Mann, Adlaf et al. 2007; Lasebikan 2010; Mann, Stoduto et al. 2010; Pulido, Barrio et al. 2011; Mu-Chen, Joanne E. et al. 2012; Li, Brady et al. 2013; Asbridge, Mann et al. 2014).

One study investigated the use of heroin and motor vehicle collision. In this survey of heroin addicts, 34\% claimed to have been involved in a motor vehicle collision when high (Edwards and Quartaro 1978) As for cocaine, a survey of adults in Ontario, Canada $(n=8,107)$, found that self-reported cocaine use was associated with a two-time increase of motor vehicle collisions (Stoduto, Mann et al. 2012). A self-reported survey conducted in Spain also found a significant increase of motor vehicle collisions among drivers who used cocaine at least once per week (Pulido, Barrio et al. 2011).

As for broad categories of drugs, two studies have been conducted (Kuypers, Legrand et al. 2012; Li, Brady et al. 2013) In a population-based case-control study conducted in the Netherlands, the risk of motor vehicle collision was highest among drivers who combined stimulants with sedatives (Kuypers, Legrand et al. 2012). In a case-control study conducted in the U.S., which used data obtained from fatal traffic crashes, those who used stimulants, depressants, narcotics, and marijuana were all at a significantly increased risk fatal motor vehicle collision while using these substances (Li, Brady et al. 2013).

\subsubsection{The effects of illicit drugs on actual driving ability}


Several studies in Europe have actually assessed the effects of certain illegal drugs on actual driving ability. The drugs that have been studied are marijuana and 3, 4-

methylenedioxymethamphetamine (MDMA). Gramekers et al performed a cross-over study among 18 subjects treated with marijuana then placebo using an instrumented vehicle in normal traffic. Marijuana significantly impaired driving performance (Gramaekers, Robbe et al. 2000). Bosker et al conducted a double-blind, placebo-controlled, randomized, three-way cross-over study among occasional $(\mathrm{n}=12)$ and heavy cannabis users $(n=12)$ using an instrumented vehicle (Bosker, Kuypers et al. 2012). Weaving within the lane and reaction time were the outcome measures. Driving performance was affected in both groups while taking marijuana (Bosker, Kuypers et al. 2012). Three studies have used cross-over designs to assess the effects of MDMA on driving performance using instrumented vehicles (Kuypers, Samyn et al. 2006; Ramaekers, Kuypers et al. 2006; Bosker, Kuypers et al. 2012). Two of the three studies investigated the effects a short time after use (Kuypers, Samyn et al. 2006; Ramaekers, Kuypers et al. 2006), while the third study tested how sleep loss mediates the drug's effects (Bosker, Kuypers et al. 2012). MDMA affected driving performance in all studies but even more when sleep loss was taken into account (Kuypers, Samyn et al. 2006; Ramaekers, Kuypers et al. 2006; Bosker, Kuypers et al. 2012).

\subsection{Challenges of studying the association between drug use and motor vehicle collisions}

The fundamental challenge of studying the association between licit or illicit drug usage and driving ability is that the relationships are not always lucid. For example, it is well-established in the literature that alcohol effects everyone's driving ability to some extent even in small doses. This is not always the case with drugs, particularly in an older adult population. There are several reasons for this. First, the relationships between aging, existing medical conditions, and drug usage are often difficult—if not impossible - to separate. It is difficult to determine exactly which is more responsible for declines in driving ability and/or motor vehicle collision risk as each can affect individuals. Second, it can be how the drugs and their effects are studied. Differences in study designs and inherent biases, such as confounding, misclassification, and selection bias, can also skew study findings (Rothman, Greenland et 
al. 2008). Lastly, drugs can elicit their effects differently amongst individuals. There is a plethora of research that shows various endogenous and exogenous factors that affect drug metabolism among individuals. Some of these factors include, but are not limited to: pharmacokinetic or pharmacodynamic properties of the drug (Jusko 2013), the drug's half life (Brown, Milavetz et al. 2013), developed tolerance (Stein and Baerwald 2014), elimination rate (Bushardt, Massey et al. 2008), dosage (Brown, Milavetz et al. 2013), route of administration (Bushardt, Massey et al. 2008), drug solubility (Augustijns, Wuyts et al. 2014), hormonal fluctuations (Berta, Harangi et al. 2014), alcohol use (Brown, Milavetz et al. 2013), age (Brzaković, Vezmar Kovačević et al. 2012), weight (Brzaković, Vezmar Kovačević et al. 2012), multiple drug use (Bushardt, Massey et al. 2008), gender (Chu 2014), diet (Cingi, Toros et al. 2013), genetics (Daly 2014), circadian rhythms (Innominato, Roche et al. 2014), disease complications (Joy, Frye et al. 2014), supplement use (Markert, Ngui et al. 2014), race/ethnicity (Spokoyny, Barazangi et al. 2014), and smoking status (Unterecker, Hiemke et al. 2012).

\subsection{Extant gaps in the literature}

As seen in this review, a scarcity of information is available in the U.S. regarding driving under the influence of drugs, particularly among senior drivers (Lotfipour and Vaca 2007). Generally, most of the research has focused on broad categories of drugs (LeRoy and Morse 2008) or medical conditions in older adults that are associated with an increased risk of motor vehicle collision (Koepsell, Wolf et al. 1994; Foley, Wallace et al. 1995; McGwin, Sims et al. 2000). To the author's knowledge, no studies have examined which specific licit, illicit, or combinations of these drugs are most common among senior drivers. Few studies have tried to assess the risk of motor vehicle collision posed by individual medications in this age group.

\subsection{Rational and purpose of study}

The overarching goal of this dissertation research is to characterize licit and illicit drug use and to explore which individual licit drugs and combinations may affect driving ability and/or the risk of motor 
vehicle collision among drivers 65 years of age and older in the U.S. The approach to investigating this issue will involve a systematic review of the literature (Chapter 2), a cross-sectional analysis of a national traffic fatality database (Chapter 3), and a case-crossover study using electronic medical records (Chapter4). Results of all three studies will be discussed in Chapter 5. The purpose of the systematic review is to systematically search published and unpublished studies pertaining to specific medication use and motor vehicle collision. This study will help to synthesize the information and help determine which individual medications are associated with driver impairment. The purpose of the cross-sectional analysis is to determine which specific and broad categories of licit and illicit drugs and subsequent combinations are most prevalent among a nationally representative population of fatally injured drivers. This study will allow the demographic characteristics to be compared between drivers who were found drug positive to those who were not. It will also allow for the comparison of prevalence rates of individual drugs and combinations through the use of a referent population of 30-50 year old drivers. The purpose of the casecrossover study is to explore which individual medication exposures may be associated with increased risk of motor vehicle collision among West Virginia drivers 65 years of age and older who were admitted for medical treatment post-collision. Because this study is utilizing electronic medical records, the study will enable population demographics to be characterized, to distinguish which licit drugs and combinations are most prevalent in this population, discern illicit drug use via laboratory test results, and to decipher the risks that certain medications pose to drivers who consume them prior to collision. 


\title{
Chapter 2
}

Medication use and the risk of motor vehicle collisions among licensed drivers: A systematic review

\begin{abstract}
Objectives: Driving under the influence of prescription and over-the-counter medication is a growing public health concern. A systematic review of the literature was performed to investigate which specific medications were associated with increased risk of motor vehicle collision (MVC).

Methods: The a priori inclusion criteria were: 1) studies published from English-language sources on or after January 1, 1960, 2) licensed drivers 15 years of age and older, 3) peer-reviewed publications, master's theses, doctoral dissertations, and conference papers, 4) studies limited to randomized control trials, cohort studies, case-control studies, or case-control type studies 5) outcome measure reported for at least one specific medication, 6) outcome measure reported as the odds or risk of a motor vehicle collision or some affected aspect of driving ability during an on the road assessment or driving simulation. The last search was conducted in June 2014. Fourteen databases (i.e. Medline, PubMed, Scopus, International Pharmaceutical Abstracts, Cochrane Central Register of Controlled Trials, CINAHL, AgeLine, Web of Science, PsychInfo, Transportation Research Information Services, Academic Search Complete, EconLit, SafetyLit and ProQuest Dissertations and Theses) were examined along with hand-searching. Independent, dual selection of studies and data abstraction was performed.
\end{abstract}

Results: After the removal of duplicate studies, 4,578 articles were identified. Of these studies, 4,134 were excluded after the first screen for eligibility. Of the 444 full text articles remaining, fifty-three medications were investigated by 27 studies included in the review. Fifteen $(28.3 \%)$ were associated with an increased risk of MVC. These included Buprenorphine, Codeine, Dihydrocodeine, Methadone, Tramadol, Levocitirizine, Diazepam, Flunitrazepam, Flurazepam, Lorazepam, Temazepam, Triazolam, Carisoprodol, Zolpidem, and Zopiclone.

Conclusions: Several medications were associated with an increased risk of MVC and decreased driving ability. The associations between specific medication use and the increased risk of MVC and/or affected driving ability are complex. Future research opportunities are plentiful and worthy of such investigation. 


\subsection{Introduction}

While the number of motor vehicle collisions (MVC) and subsequent fatalities has steadily declined over the past decade (Sise, Calvo et al. 2014), MVC still remains one of the leading causes of mortality in the United States (U.S.) (Rockett, Regier et al. 2012). In 2010, this equated to approximately one death per collision every fifteen minutes (Oster and Strong 2013). Besides the inherent risks to morbidity and mortality, MVCs are estimated to cost the U.S. over $\$ 300$ billion dollars per year (Oster and Strong 2013).

It is well-established in the literature that alcohol can negatively influence driving ability, even when consumed in small quantities. It is estimated that over one-third of all MVC are a result of driver intoxication (Hayman and Crandall 2009). While driving under the influence of alcohol is a welldocumented area of study, driving under the influence of drugs (DUID) is also an emerging public health and traffic safety concern (Morland 2000; Movig, Mathijssen et al. 2004; Walsh, de Gier et al. 2004). Driving under the influence of drugs entails the use of illicit drugs, i.e. drugs that are obtained illegally and with no real medical benefit, such as cocaine and methamphetamine. Driving under the influence of drugs can also entail the use of licit substances, such as common prescription or over-the-counter medications, whose effects impair the driver's ability to safely operate a motor vehicle from one destination to another. However, it's important to realize that while licit drugs can be obtained illegally, abused, or misused, the intent of use by the driver is often difficult to determine. In 2009, approximately $28 \%$ of all fatally injured U.S. drivers that were tested for either illicit or licit drugs tested positive for one or more of these substances (National Highway Traffic Safety Administration 2010). In addition, recent research suggests that DUID is increasing nationally (Wilson, Stimpson et al. 2014).

Due to the complexity of DUID, the primary focus of this paper pertains to the association between licit drug use and MVC. However, one of the fundamental challenges to studying the effects of licit drugs on driving ability is that the relationship is not always as apparent when compared to alcohol (National Highway Traffic Safety Administration 2010). For example, some drugs may not noticeably impair the skills (cognition, psychomotor function, physical ability) necessary to operate a motor vehicle 
(Coopersmith, Korner-Bitensky et al. 1989; Carr 2000; Carr, Duchek et al. 2006; Cheung and McCartt 2011). Drugs that are perceived to affect the central nervous system may exhibit different effects among individuals; this may be attributed to the pharmacokinetic or pharmacodynamic properties of the drug (Jusko 2013), the drug's half-life (Brown, Milavetz et al. 2013), interactions with other consumed drugs (Bushardt, Massey et al. 2008), tolerance (Stein and Baerwald 2014), drug elimination rate (Bushardt, Massey et al. 2008), dosage (Brown, Milavetz et al. 2013), route of administration (Bushardt, Massey et al. 2008), solubility (Augustijns, Wuyts et al. 2014), intestinal pH (Augustijns, Wuyts et al. 2014), current health status of the individual (Bushardt, Massey et al. 2008), genetics (Daly 2014), etc. It may also be difficult to partition out the effects of the licit drug and the medical condition for which it was taken to remedy (Bushardt, Massey et al. 2008). For example, several medical conditions have been associated with an increased risk of MVC. These include, but are not necessarily limited to, sleep apnea (Ellen, Marshall et al. 2006), dementia (Brown and Ott 2004), arthritis (Cross, McGwin et al. 2009), diabetes (Hansotia and Broste 1991), epilepsy (Hansotia and Broste 1991), anxiety (Sagberg 2006), depression (Sagberg 2006) and Parkinson's disease (Uc, Rizzo et al. 2006).

Numerous reviews and meta-analyses have investigated the association between licit drug use and MVC and/or driving ability. These reviews have focused predominately on opioids (Fishbain, Cutler et al. 2002; Fishbain, Cutler et al. 2003; Kress and Kraft 2005; Borgeat 2010; Leung 2011; Jones, Mogali et al. 2012; Mailis-Gagnon, Lakha et al. 2012; Strand, Fjeld et al. 2013; Soyka 2014), benzodiazepines (van Laar and Volkerts 1998; Rapoport, Lanctôt et al. 2009; Smink, Egberts et al. 2010; Dassanayake, Michie et al. 2011; Leung 2011; Jones, Mogali et al. 2012), antihistamines (Roberts 2005; Popescu 2008) psychoactive drugs (Rapoport and Baniña 2007; Joris C. and Monique Anna Johanna 2009; Verster and Mets 2009; Krueger 2010; Cooper, Meuleners et al. 2011), antidepressants (Ramaekers 2003; Verster and Ramaekers 2009; Dassanayake, Michie et al. 2011; Ravera, Ramaekers et al. 2012; Brunnauer and Laux 2013), hypnotics (Verster, Veldhuijzen et al. 2006; Krueger 2010), anxiolytics (Verster, Veldhuijzen et al. 2005; Vermeeren, Leufkens et al. 2009) and sleep medications (Verster, Volkerts et al. 2007; Verster, Volkerts et al. 2007; Gunja 2013; Leufkens and Vermeeren 2014). Some reviews have also examined 
multiple drug categories (Kelly, Darke et al. 2004; Orriols, Salmi et al. 2009; Elvik 2013; Amanda and David B. 2014). However, the majority of these studies have reviewed or analyzed licit drugs in broad groups (Fishbain, Cutler et al. 2002; Kress and Kraft 2005; Joris C. and Monique Anna Johanna 2009; Rapoport, Lanctôt et al. 2009; Dassanayake, Michie et al. 2011; Leung 2011; Jones, Mogali et al. 2012; Ravera, Ramaekers et al. 2012). There is the potential that if the drugs within these groups were reviewed individually, the outcome measures of interest may be varied as some drugs may be more or less driverimpairing than others. Therefore, the purpose of this study was to perform a systematic review of the literature to investigate which specific medications, including typical prescription or over-the-counter drugs, may be associated with an increased risk or odds of MVC and/or driving ability among licensed drivers 15 years of age and older.

\subsection{Methods}

\section{Study eligibility}

The inclusion criteria for studies, which were defined a priori, were as follows: 1) Englishlanguage studies published on or after January 1, 1960,2) licensed drivers 15 years of age and older, 3) studies published in a peer-reviewed journal or non-published (i.e. "grey literature"), which included master's theses, doctoral dissertations, and conference papers, 4) studies limited to randomized control trials, cohort studies, case-control studies, or case-control types of studies, i.e. case cross-over, case-time series, etc. 5) outcome measure reported for at least one specific medication, 6) outcome measure reported as the odds or risk of a motor vehicle collision or some affected aspect of driving ability during an onroad assessment or driving simulation (e.g. brake reaction time, weaving, standard deviation of lateral position, etc.). If the study reported outcome measures for both specific medications and illicit drugs or specific medications combined with alcohol, only outcome measures for specific medications alone were reported. A 'medication' was defined as a substance either available by prescription or over-the-counter to remedy a medical condition. Therefore, caffeine, nicotine, vitamins, and nutraceuticals were excluded. If the medication usage was combined with a medical procedure, then the study was excluded to avoid bias. While marijuana has been legalized for medicinal purposes in several states, it was not considered a 
medication in this analysis as it is still defined as an illegal substance by federal law. Because of the vast difference in the fidelity of driving simulators, a driving simulator must have consisted of a screen, pedals, and steering wheel. If the study did not specify the components of the simulator, an attempt was made to search the make and model of the simulator noted in the study to see if it was comprised of these constituents. The search date of January 1, 1960 was arbitrarily chosen as no DUID studies existed or were published prior to this time. Because of the complexity of the initial study question, it was decided post-hoc to only present the studies whose outcomes reported the association between a specific medication and the odds or risk of a motor vehicle collision.

\section{Data sources}

Studies were acquired from the following fourteen databases: 1) Medline (within EBSCO host), 2) PubMed, 3) Scopus, 4) International Pharmaceutical Abstracts (IPA), 5) Cochrane Central Register of Controlled Trials (CENTRAL), 6) CINAHL (within EBSCO host), 7) AgeLine, 8) Web of Science (WOS), 9) PsychInfo, 10) Transportation Research Information Services (TRID), 11) Academic Search Complete, 12) EconLit 13) SafetyLit, and 14) ProQuest Dissertations and Theses (ProQuest). All searches were performed by TMR with the assistance of a Health Sciences Librarian from West Virginia University. The last search was performed in June 2014. All searches were conducted using Medical Subject Headings (MESH) terminology. Each search contained the phrases, "drug”, "medication", "traffic collision", and "motor vehicle". In addition to the fourteen databases, studies from TMR's personal library were also reviewed for eligibility. Hand searches from the reference lists of included studies were also examined. Government websites, such as the National Highway Traffic Safety Administration, were also searched for relevant government-performed studies.

\section{Selection of studies}

All included studies were independently selected by TMR and BR. Any discrepancies regarding the inclusion of studies were resolved through discussion. If a consensus could not be reached, MZ acted as the arbitrator. All studies, whether included or excluded in the review, were stored in EndNote, version X5, along with reasons for including or excluding the study. 


\section{Data abstraction}

A codebook was developed by TMR in Microsoft Excel 2007. The codebook included variables regarding study characteristics, study population, medications investigated, outcome measures, and study quality. All studies were coded by TMR and CP independent of one another. After coding was completed, both authors met to compare the entries for accuracy and/or precision. Any discrepancies were resolved through discussion. If disagreements could not be rectified, MZ acted as the arbitrator.

\section{Study quality}

The included studies were evaluated for quality by TMR and CP independent of one another. An abbreviated version of the checklist for measuring study quality designed by Downs and Black was used to score the studies for quality (Downs and Black 1998). The original checklist designed by Downs and Black, which has been deemed both valid and reliable, is a 27 question, qualitative check-list to evaluate a study for internal and external validity, quality of reporting, and power; for every positive attribute/response, a study receives one point (Downs and Black 1998). At the end of assessment, the points are tallied; the more points a study earns, the higher the study's quality (Downs and Black 1998). After all coding and quality assessments were completed, the authors met to compare their entries for accuracy and/or precision. Any discrepancies were resolved through discussion. If disagreements could not be reached, MZ acted as the arbitrator. No studies that met the eligibility criteria were excluded based on quality.

\section{Data synthesis}

All studies were synthesized qualitatively. No meta-analysis was performed given the a priori assumption that excessive statistical and/or methodological heterogeneity would be observed (Purgato and Adams 2012). However, the overall odds or risk ratio for each medication and outcome was presented graphically and included the point estimate and corresponding 95\% confidence intervals. Post hoc, it was realized that some included studies only presented crude estimates (Nelson 1992; Koepsell, Wolf et al. 1994; Neutel 1998; Cui 2001; Mura, Kintz et al. 2003; Mathijssen 2006; Bramness, Skurtveit et al. 2007; Gustavsen, Bramness et al. 2008; Lonnen, Powell et al. 2008; Bachs, Engeland et al. 2009; 
Bramness, Skurtveit et al. 2009; Gibson, Hubbard et al. 2009; Skurtveit, Strøm et al. 2009; Coupland, Dhiman et al. 2011; Gjerde, Normann et al. 2011; Orriols, Philip et al. 2011; Bramness, Skurtveit et al. 2012; Corsenac, Lagarde et al. 2012; Hansen 2012; Gjerde, Christophersen et al. 2013), while others adjusted their outcome measures for various potential confounders such as age, gender, miles driven, day of the week, etc (Foley, Wallace et al. 1995; McGwin, Sims et al. 2000; Sims, McGwin et al. 2000; Etminan, Hemmelgarn et al. 2004; Delaney, Opatrny et al. 2006; Hemmelgarn, Lévesque et al. 2006; Yang, Lai et al. 2011). For those studies that presented adjusted estimates or did not include 95\% confidence intervals for crude estimates (Foley, Wallace et al. 1995; McGwin, Sims et al. 2000; Sims, McGwin et al. 2000; Etminan, Hemmelgarn et al. 2004; Delaney, Opatrny et al. 2006; Hemmelgarn, Lévesque et al. 2006; Yang, Lai et al. 2011), then the adjusted point estimates and 95\% confidence intervals for the population were reported. If a study's point estimate was greater than one, this indicated that there was excess risk of collision; a point estimate less than one indicated that the medication use was associated with a decreased risk of collision (i.e. it was protective against MVC). Results were considered statistically significant if the $95 \%$ confidence intervals did not include one.

\subsection{Results}

\section{Study characteristics}

The search processes for the selection of studies, as well as reasons for excluding studies, are presented in Figure 1. Of the 6,516 records obtained, 208 studies met the original study question. Of these 208 studies, 27 pertained to the association of specific medications and the odds or risk of motor vehicle collision, while the others pertained to the association of specific medications and affected driving ability determined through the use of driving simulators $(n=90)$ or actual driving assessments $(n=91)$. The characteristics of the 27 studies included in this review are presented in Table 1 (Nelson 1992; Koepsell, Wolf et al. 1994; Foley, Wallace et al. 1995; Neutel 1998; McGwin, Sims et al. 2000; Sims, McGwin et al. 2000; Cui 2001; Mura, Kintz et al. 2003; Etminan, Hemmelgarn et al. 2004; Delaney, Opatrny et al. 2006; Hemmelgarn, Lévesque et al. 2006; Mathijssen 2006; Bramness, Skurtveit et al. 2007; Gustavsen, Bramness et al. 2008; Lonnen, Powell et al. 2008; Bachs, Engeland et al. 2009; 
Bramness, Skurtveit et al. 2009; Gibson, Hubbard et al. 2009; Skurtveit, Strøm et al. 2009; Coupland, Dhiman et al. 2011; Gjerde, Normann et al. 2011; Orriols, Philip et al. 2011; Yang, Lai et al. 2011; Bramness, Skurtveit et al. 2012; Corsenac, Lagarde et al. 2012; Hansen 2012; Gjerde, Christophersen et al. 2013). The included studies spanned from 1992-2013. Of these studies, eight (29.6\%) were conducted in Norway (Bramness, Skurtveit et al. 2007; Gustavsen, Bramness et al. 2008; Bachs, Engeland et al. 2009; Bramness, Skurtveit et al. 2009; Skurtveit, Strøm et al. 2009; Gjerde, Normann et al. 2011; Bramness, Skurtveit et al. 2012; Gjerde, Christophersen et al. 2013), six (22.2\%) in the U.S. (Nelson 1992; Koepsell, Wolf et al. 1994; Foley, Wallace et al. 1995; McGwin, Sims et al. 2000; Sims, McGwin et al. 2000; Hansen 2012), five (18.5\%) from Canada (Neutel 1998; Cui 2001; Etminan, Hemmelgarn et al. 2004; Delaney, Opatrny et al. 2006; Hemmelgarn, Lévesque et al. 2006), three (11.1\%) from England (Lonnen, Powell et al. 2008; Gibson, Hubbard et al. 2009; Coupland, Dhiman et al. 2011), three (11.1\%) from France (Mura, Kintz et al. 2003; Orriols, Philip et al. 2011; Corsenac, Lagarde et al. 2012), one (3.7\%) from the Netherlands (Mathijssen 2006), and one (3.7\%) from Taiwan (Yang, Lai et al. 2011). Eleven (40.7\%) of these studies were cohort designs, while the rest consisted of case-control or variations of a case-control design. Only one study was limited to females (Nelson 1992). Nine $(33.3 \%)$ of the studies were limited to older adult drivers typically 60 years of age and older, nine (33.3\%) included drivers aged approximately 18-70 years, and the remaining nine (33.3\%) covered all licensed drivers from the country of origin. Three studies $(11.1 \%)$ were doctoral dissertations (Nelson 1992; Cui 2001; Hansen 2012), one study (3.7\%) was a conference paper (Mathijssen 2006), and the remaining 23 studies (85.2\%) were published in peer-reviewed journals. Among these studies, 53 specific medications were evaluated. Diazepam, Zolpidem, Zopiclone, and Insulin were the most commonly studied medications. With respect to study quality, 23 of the 27 studies (85.2\%) received a score of 5 (Table 1). The studies that were scored 4 out of 5 typically did not explain their statistical methodology in sufficient detail, i.e., did not mention or explain what types of regression they utilized to obtain study outcome measurements (Neutel 1998; Mura, Kintz et al. 2003; Mathijssen 2006). 


\section{Study outcomes}

The medications that were investigated by the studies included in this review are presented by drug category in Figures 2-9. The drug categories consisted of analgesics, anticonvulsants, antidepressants, antihistamines, antihyperglycemics, benzodiazepines, and sleep-enhancing medications; if a drug could not be categorized into any of these groups, it classified as 'Other Medications'. Studies were adjusted by the original study authors for age and gender (Foley, Wallace et al. 1995), age, sex, gender, and annual mileage (McGwin, Sims et al. 2000), age, sex, gender, and days driven per week (Sims, McGwin et al. 2000), and age, gender, residence in the country, previous injurious MVC, chronic disease score, exposure to antidepressants, anti-epileptics, benzodiazepines, antipsychotics, anti-migraine, muscle relaxants, and narcotic analgesics (Etminan, Hemmelgarn et al. 2004). Another study was adjusted for cardiac or stroke events within the past year as well as the following drug classes in the previous 60 days: antidepressants, anti-epileptics, benzodiazepines, antipsychotics, anti-migraine, narcotic analgesics, muscle relaxants (Delaney, Opatrny et al. 2006). Another study was adjusted for age, gender, previous MVC, and place of residence (Hemmelgarn, Lévesque et al. 2006), while another was adjusted for concomitant use of the following medications: Zolpidem, Zopiclone, long and short acting benzodiazepines, antihistamines, anticonvulsants, antidepressants, other sedatives/hypnotics, other psychoactive drugs, muscle relaxants, and opioid analgesics (Yang, Lai et al. 2011).

\section{Analgesics}

Among the included studies, the associations between seven analgesic medications and the odds or risk of MVC were investigated (Figure 2). These medications included Aspirin (Foley, Wallace et al. 1995), Buprenorphine (Corsenac, Lagarde et al. 2012), Codeine (Mathijssen 2006; Bachs, Engeland et al. 2009; Gibson, Hubbard et al. 2009), Dihydrocodeine (Gibson, Hubbard et al. 2009), Methadone (Bramness, Skurtveit et al. 2012; Corsenac, Lagarde et al. 2012), Morphine (Gibson, Hubbard et al. 2009), and Tramadol (Bachs, Engeland et al. 2009; Gibson, Hubbard et al. 2009). As shown in Figure 2, those taking Aspirin (Foley, Wallace et al. 1995) and Morphine (Gibson, Hubbard et al. 2009) did not experience a statistically significant increase in MVC; contrarily, those taking Buprenorphine (Corsenac, 
Lagarde et al. 2012), Dihydrocodeine (Gibson, Hubbard et al. 2009), and Methadone (Bramness, Skurtveit et al. 2012; Corsenac, Lagarde et al. 2012) did experience an increase in MVC risk. The results for both Codeine and Tramadol were mixed. Three studies investigated the effects of Codeine on the risk of MVC; two of these studies reported a statistically significant increase in MVC (Bachs, Engeland et al. 2009; Gibson, Hubbard et al. 2009), while one study did not (Mathijssen 2006). The study that did not report an association for the increased risk of MVC and Codeine may be because of sample size (Mathijssen 2006); the study may not have had the statistical power to detect a difference. As for Tramadol, two studies investigated its effects and only one (Gibson, Hubbard et al. 2009) reported a statistically significant increase in MVC. This, too, may have been attributed to sample size. Out of 181 collisions, only 20 people were found positive for Tramadol in the study conducted by Bachs et al (Bachs, Engeland et al. 2009).

\section{Anticonvulsants}

Three studies investigated the effects of anticonvulsants, which included Carbamazepine (Etminan, Hemmelgarn et al. 2004), Phenytoin (Nelson 1992), and Valproate (Bramness, Skurtveit et al. 2009). As seen in Figure 3, none of these medications were associated with a statistically significant increase of MVC (Nelson 1992; Etminan, Hemmelgarn et al. 2004; Bramness, Skurtveit et al. 2009). Though not significant, Carbamazepine and Valproate were trending towards being protective against the risk of MVC.

\section{Antidepressants}

Antidepressant medications were the most studied class of drug. Twelve medications were investigated amongst three studies, which included Amitriptyline (Gibson, Hubbard et al. 2009; Coupland, Dhiman et al. 2011), Cetirizine (Gibson, Hubbard et al. 2009), Citalopram (Gibson, Hubbard et al. 2009; Coupland, Dhiman et al. 2011), Doselupin (Gibson, Hubbard et al. 2009; Coupland, Dhiman et al. 2011), Escitalopram (Coupland, Dhiman et al. 2011), Fluoxetine (Gibson, Hubbard et al. 2009; Coupland, Dhiman et al. 2011), Lofepramine (Gibson, Hubbard et al. 2009; Coupland, Dhiman et al. 2011), Mirtazapine (Coupland, Dhiman et al. 2011), Paroxetine (Gibson, Hubbard et al. 2009; Coupland, 
Dhiman et al. 2011), Sertraline (Coupland, Dhiman et al. 2011), Trazodone (Gibson, Hubbard et al. 2009; Coupland, Dhiman et al. 2011; Hansen 2012) and Venlafaxine (Coupland, Dhiman et al. 2011). As seen in Figure 4, with the exception of one outcome for Doselupin and two for Trazodone, virtually all of these medications were found to not be associated with a statistically significant increase of MVC. Though not significant, several of these medications were trending towards being protective against a MVC; this included Amitriptyline, Fluoxetine, Mirtazapine, Paroxetine, Sertraline, and Venlafaxine. In one study, Doselupin was found protective (Coupland, Dhiman et al. 2011) against MVC while another study (Gibson, Hubbard et al. 2009) found no association. This may be attributed to the differences in study design or possibly the study population as the study that found Doselupin protective was limited to older adult drivers (Coupland, Dhiman et al. 2011). All three studies investigated Trazodone and the findings for this medication were mixed. Two studies found Trazodone to not be statistically significant for the increased risk of MVC (Gibson, Hubbard et al. 2009; Coupland, Dhiman et al. 2011). Hansen found that both new $(\mathrm{HR}=2.15 ; 95 \% \mathrm{CI} 1.83-2.51)$ and prevalent users (HR=1.47; $95 \%$ CI 1.18-1.85) of Trazodone to be at increased risk of MVC (Hansen 2012). These differences may be attributed to the study population or possibly the study design.

\section{Antihistamines}

One study investigated the effects of nine antihistamines on the odds of MVC, which included Astemizole, Brompheniramine, Chlorphenamine, Desloratidine, Fexofenadine, Hydroxyzine, Levocitirizine, Loratadine, and Terfenadine (Gibson, Hubbard et al. 2009). As seen in Figure 5, only Levocitirizine was associated with an increased risk of MVC (Gibson, Hubbard et al. 2009).

\section{Antihyperglycemics}

Six studies investigated three antihyperglycemic medications which included Insulin (McGwin, Sims et al. 2000; Sims, McGwin et al. 2000; Cui 2001; Hemmelgarn, Lévesque et al. 2006; Lonnen, Powell et al. 2008; Skurtveit, Strøm et al. 2009), Metformin (Hemmelgarn, Lévesque et al. 2006), and Sulfonylurea (Hemmelgarn, Lévesque et al. 2006) (Figure 6). Of the six studies that reported outcome measures for Insulin, only two reported a statistically significant increase in MVC (Cui 2001; Skurtveit, 
Strøm et al. 2009). The other four studies reported no statistically significant associations (McGwin, Sims et al. 2000; Sims, McGwin et al. 2000; Hemmelgarn, Lévesque et al. 2006; Lonnen, Powell et al. 2008). This may be attributed to the adjustment of covariates. One study which included the odds of having any crash or a not-at-fault crash was adjusted for age, sex, race, and annual mileage (McGwin, Sims et al. 2000), while another study was adjusted for age, gender, and days driven per week (Sims, McGwin et al. 2000). Other studies were adjusted for age, sex, previous MVC, and place of residence (Hemmelgarn, Lévesque et al. 2006), or unadjusted but stratified by the following ages: <25, 25-44, 4564, 65-74, and 75-84 years of age (Lonnen, Powell et al. 2008). Since two studies were not adjusted and the other studies were adjusted or stratified by age, it is possible that these two studies were statistically significant because they were left unadjusted (Cui 2001; Skurtveit, Strøm et al. 2009). In addition, one study found that Metformin was not associated with an increased risk of crash, while Sulfonylurea was protective against MVC (Hemmelgarn, Lévesque et al. 2006).

\section{Benzodiazepines}

Nine benzodiazepines were investigated amongst seven studies which included Chlordiazepoxide (Gibson, Hubbard et al. 2009), Diazepam (Neutel 1998; Bramness, Skurtveit et al. 2007; Gibson, Hubbard et al. 2009; Gjerde, Normann et al. 2011; Gjerde, Christophersen et al. 2013), Flunitrazepam (Gustavsen, Bramness et al. 2008), Flurazepam (Neutel 1998), Lorazepam (Neutel 1998), Nitrazepam (Gustavsen, Bramness et al. 2008; Gibson, Hubbard et al. 2009), Oxazepam (Neutel 1998), Temazepam (Gibson, Hubbard et al. 2009; Hansen 2012), and Triazolam (Neutel 1998) (Figure 7). Chlordiazepoxide and Oxazepam were not found to be statistically significant for the increased risk of MVC (Neutel 1998; Gibson, Hubbard et al. 2009). Flunitrazepam, Flurazepam, Lorazepam, Temazepam, and Triazolam were found to be statistically significant for an increased risk of MVC (Neutel 1998; Gustavsen, Bramness et al. 2008; Gibson, Hubbard et al. 2009). The findings for Diazepam were slightly mixed. Of the five studies investigating the effects of Diazepam, four found a statistically significant increase in MVC (Neutel 1998; Bramness, Skurtveit et al. 2007; Gibson, Hubbard et al. 2009; Gjerde, Christophersen et al. 2013), while only one did not (Gjerde, Normann et al. 2011). Since the confidence intervals for one study 
was quite large, this estimate was likely not precise (Gjerde, Normann et al. 2011). The findings were also mixed for Nitrazepam. One study found it to be associated with an increased risk of MVC (Gustavsen, Bramness et al. 2008), while another did not (Gibson, Hubbard et al. 2009). It should be noted that the study that did not report a statistically significant increase in MVC trended towards an increased risk (Gibson, Hubbard et al. 2009). This discrepancy may have been attributed to different study designs.

\section{Sleep Medications}

Zolpidem and Zopiclone were the only two strictly sleep-enhancing medications to be investigated (Figure 8). Five studies explored Zolpidem's effects (Gustavsen, Bramness et al. 2008; Gibson, Hubbard et al. 2009; Orriols, Philip et al. 2011; Yang, Lai et al. 2011; Hansen 2012), while six studies examined Zopiclone (Gustavsen, Bramness et al. 2008; Gibson, Hubbard et al. 2009; Gjerde, Normann et al. 2011; Orriols, Philip et al. 2011; Yang, Lai et al. 2011; Gjerde, Christophersen et al. 2013). Both medications appeared to be associated with an increased risk of MVC. Four (Gustavsen, Bramness et al. 2008; Orriols, Philip et al. 2011; Yang, Lai et al. 2011; Hansen 2012) of five studies found Zolpidem to be statistically significant for an increased risk of MVC. Two (Gustavsen, Bramness et al. 2008; Gjerde, Normann et al. 2011) of six studies found Zopiclone to be statistically significant for an increased risk of MVC, while the other four were trending towards an increased risk, but not statistically significant (Gibson, Hubbard et al. 2009; Orriols, Philip et al. 2011; Yang, Lai et al. 2011; Gjerde, Christophersen et al. 2013).

\section{Other Medications}

Eight medications could not be grouped into any of the other drug categories (Figure 9). These included Atenolol (i.e. a beta-blocker) (Gibson, Hubbard et al. 2009), Carisoprodol (i.e. a muscle relaxant) (Bramness, Skurtveit et al. 2007), Estrogen (i.e. a hormone) (Sims, McGwin et al. 2000), Lithium (i.e. an antipsychotic) (Etminan, Hemmelgarn et al. 2004; Bramness, Skurtveit et al. 2009), Methyldopa (i.e. an antihypertensive) (Nelson 1992), Propranolol (i.e. a beta-blocker) (Gibson, Hubbard et al. 2009), Salbutamol (i.e. an antispasmodic) (Bramness, Skurtveit et al. 2007), and Warfarin (i.e. an 
anticoagulant) (Delaney, Opatrny et al. 2006). Of these medications, Carisoprodol was associated with a statistically significant increase in MVC (Bramness, Skurtveit et al. 2007). The findings for Lithium and Warfarin were mixed. One study reported an increased risk of MVC for Lithium (Etminan, Hemmelgarn et al. 2004), while another reported no statistically significant association (Bramness, Skurtveit et al. 2009). This may have been attributed to differences in study design or the age of the population. As for Warfarin, one study found that Warfarin was initially protective against MVC for new users, but if taken for an extended period of time, no association was found (Delaney, Opatrny et al. 2006). All other medications were not found to be statistically significant for the increased risk of MVC.

\subsection{Discussion}

\section{Findings}

The principal finding of this study is that among the 53 specific medications investigated by the 27 studies included in this review, 15 medications (28.3\%) were associated with an increased risk of motor vehicle collision. The medications that were associated with an increased risk of collision were: Buprenorphine, Codeine, Dihydrocodeine, Methadone, Tramadol, Levocitirizine, Diazepam, Flunitrazepam, Flurazepam, Lorazepam, Temazepam, Triazolam, Carisoprodol, Zolpidem, and Zopiclone. Two (3.8\%) of the 53 medications, Sulfonylurea and Warfarin, may be protective against MVC. All other 36 medications (67.9\%) were not significantly associated with MVC. The findings of this review illustrate that certain medications, even within the same class or drug category, may be more associated with crash risk than others.

These findings are consistent with the current literature. With the exception of Carisoprodol, Dihydrocodeine, and Tramadol, 12 of the 15 medications that were associated with increased MVC have also been studied in either driving simulations or actual driving assessments. Both types of studies use common measures to assess one's ability to safely navigate a vehicle; these measures include the ability to maintain position within the lane [i.e. standard deviation of lateral position (SDLP)], tracking (i.e. following an object), missed instructions or directions, actual collisions with cars or objects, the ability to maintain constant speed, reactions or reaction time to stimuli, the ability to keep the vehicle on the road, 
steering wheel angle, divided attention (i.e. performing a task, such as acknowledging a sign or symbol, while driving), and gap acceptance (i.e. ability to judge distance between objects). There is evidence from both driving simulation and driving assessment studies that eight of these medications may also affect some aspects of driving ability; this includes Diazepam, Flunitrazepam, Flurazepam, Lorazepam, Temazepam, Triazolam, Zolpidem, and Zopiclone.

Diazepam, an anxiolytic, has been studied more extensively than any other of the medications. Six driving assessments (Biehl 1979; de Gier, t Hart et al. 1981; O'Hanlon, Haak et al. 1982; De Gier 1984; van Laar, Volkerts et al. 1992; O'Hanlon, Vermeeren et al. 1995) and ten simulation studies (Linnoila and Hakkinen 1974; Moskowitz and Smiley 1982; Willumeit, Ott et al. 1984; Willumeit, Ott et al. 1984; Smiley and Moskowitz 1986; Friedel, Joo et al. 1991; Kuitunen 1994; Vanakoski, Mattila et al. 2000; Takahashi, Iwamoto et al. 2010; Tamaji, Iwamoto et al. 2012) have investigated its effects on driving ability. The majority of studies have been randomized, double-blind, and cross-over by design. Participants in these studies have involved both genders of different age ranges. Professional drivers have also been used to study its effects (O'Hanlon, Haak et al. 1982). Diazepam has been shown to negatively affect driving ability in virtually all the studies whether the source is an actual driving assessment or driving simulation. Collectively, Diazepam has affected nearly every aspect of driving ability including braking, SDLP, incidence of collisions, driver behavior, tracking, steering, car following, and reaction time, compared to placebo conditions (Linnoila and Hakkinen 1974; Biehl 1979; de Gier, t Hart et al. 1981; Moskowitz and Smiley 1982; O'Hanlon, Haak et al. 1982; De Gier 1984; Willumeit, Ott et al. 1984; Willumeit, Ott et al. 1984; Smiley and Moskowitz 1986; Friedel, Joo et al. 1991; van Laar, Volkerts et al. 1992; Kuitunen 1994; O'Hanlon, Vermeeren et al. 1995; Vanakoski, Mattila et al. 2000; Takahashi, Iwamoto et al. 2010; Tamaji, Iwamoto et al. 2012).

Flunitrazepam, a benzodiazepine, has been studied in one simulation (Meskali, Berthelon et al. 2009) and one driving assessment (Schmidt, Brendemühl et al. 1986). In the simulation study, 16 healthy subjects underwent a double blind cross-over study where they took Flunitrazepam the night before than completed the simulation the next morning to assess residual effects of the drug. Participants tended to 
speed when taking Flunitrazepam (Meskali, Berthelon et al. 2009). In the driving assessment, 32 outpatients with sleep disorders received either $2 \mathrm{mg}$ of Flunitrazepam or $20 \mathrm{mg}$ of Temazepam daily for seven days and partook in a driving test on day one and day seven (Schmidt, Brendemühl et al. 1986). Steering, lateral acceleration, and velocity were used to assess driving ability. Those taking Flunitrazepam experienced greater decreases in steering ability compared to Temazepam (Schmidt, Brendemühl et al. 1986).

Flurazepam, a benzodiazepine derivative, has been investigated in two driving assessments (Betts and Birtle 1982; Brookhuis, Volkerts et al. 1990) and two simulations (Willumeit, Neubert et al. 1983; Willumeit, Ott et al. 1984). In a blinded, cross-over trial, 12 female volunteers received placebo or Flurazepam the night before and then drove a course the following morning. Under Flurazepam, participants incorrectly performed passable gaps (Betts and Birtle 1982). In another double-blind, crossover study, 16 patients treated for insomnia performed a $75 \mathrm{~km}$ driving assessment. When treated with Flurazepam, SDLP was decreased, but was more pronounced in female subjects during morning assessments (Brookhuis, Volkerts et al. 1990). In a double-blind, cross-over simulation using 12 healthy volunteers, general reaction time to signals was impaired under Flurazepam compared to control periods (Willumeit, Neubert et al. 1983). In another placebo-controlled, double-blind simulation using 54 healthy volunteers, executed tasks while driving were decreased in those taking Flurazepam compared to placebo periods (Willumeit, Ott et al. 1984).

Lorazepam, a benzodiazepine, has been evaluated in seven driving assessments (Hindmarch and Gudgeon 1980; Irving and Jones 1992; O'Hanlon, Vermeeren et al. 1995; Mercier-Guyon, Lejay et al. 1999; van Laar, Volkerts et al. 2001; Daurat, Sagaspe et al. 2013; Moták, Bayssac et al. 2014) and one simulation (Mattila, Kuitunen et al. 1993). All of the studies were randomized, double-blind, and crossover by design. Four of the driving assessments were limited to males (Mercier-Guyon, Lejay et al. 1999; van Laar, Volkerts et al. 2001; Daurat, Sagaspe et al. 2013; Moták, Bayssac et al. 2014), two to females (Hindmarch and Gudgeon 1980; Irving and Jones 1992), and one to a group of clinically anxious and non-anxious participants of mixed gender (O'Hanlon, Vermeeren et al. 1995). The simulation study 
encompassed healthy volunteers (Mattila, Kuitunen et al. 1993). Driving ability (i.e. SDLP, inappropriate line crossings, car handling, lane-keeping, speed, following distance, and tracking) was affected in all driving assessments and simulations (Hindmarch and Gudgeon 1980; Irving and Jones 1992; Mattila, Kuitunen et al. 1993; O'Hanlon, Vermeeren et al. 1995; Mercier-Guyon, Lejay et al. 1999; van Laar, Volkerts et al. 2001; Daurat, Sagaspe et al. 2013; Moták, Bayssac et al. 2014).

Temazepam, a benzodiazepine prescribed to treat short-term insomnia, has been evaluated in five driving assessments (Betts and Birtle 1982; O'Hanlon and Volkerts 1986; Schmidt, Brendemühl et al. 1986; Riedel, Quasten et al. 1988; Leufkens and Vermeeren 2009) and one simulation study (Partinen, Hirvonen et al. 2003). All of the driving assessments and simulations were randomized, double-blind, and cross-over by design. The driving assessments used female volunteers in two studies (Betts and Birtle 1982; O'Hanlon and Volkerts 1986), one used rotating shift workers (Riedel, Quasten et al. 1988), one used healthy volunteers (Leufkens and Vermeeren 2009), and one used insomniacs (Schmidt, Brendemühl et al. 1986). The driving simulation used only female participants (Partinen, Hirvonen et al. 2003). Temazepam affected driving ability (i.e. participants hit more bollards) in only one study, but none of the others (Betts and Birtle 1982).

Triazolam, a sedating benzodiazepine used to treat severe insomnia, has been studied in three driving assessments (Laurell and Tornros 1986; Riedel, Quasten et al. 1988; Riedel 1991) and three driving simulations (Deits, Boyle et al. 2011; Morrison, Boyle et al. 2011; Brown, Milavetz et al. 2013). All three driving assessments were randomized, double-blind, and cross-over by design (Laurell and Tornros 1986; Riedel, Quasten et al. 1988; Riedel 1991). Two of the studies used rotating shift workers (Riedel, Quasten et al. 1988; Riedel 1991), while the third used healthy volunteers (Laurell and Tornros 1986). In the two driving assessments using shift workers, Triazolam severely affected driving ability (Riedel, Quasten et al. 1988; Riedel 1991); driving performance was not as affected among the healthy volunteers (Laurell and Tornros 1986). In the simulation studies, all three were similar in design and used commercial bus drivers as the study population (Deits, Boyle et al. 2011; Morrison, Boyle et al. 2011; Brown, Milavetz et al. 2013). Driving ability (i.e. lane keeping, driving path, steering, braking, and 
SDLP) was affected in all of the simulation studies simulations (Deits, Boyle et al. 2011; Morrison, Boyle et al. 2011; Brown, Milavetz et al. 2013).

Zolpidem and Zopiclone, two sleep-promoting medications, were evaluated simultaneously in two driving assessments (Leufkens, Lund et al. 2009; Verster, Bervoets et al. 2014) and four driving simulations (Bocca, Le Doze et al. 1999; Staner, Ertlé et al. 2005; Meskali, Berthelon et al. 2009; Bocca, Marie et al. 2011). The two driving assessments were randomized, double-blind, and cross-over by design that used healthy participants of all age ranges (Leufkens, Lund et al. 2009; Verster, Bervoets et al. 2014). In both studies, Zopiclone and Zolpidem affected driving ability. In the simulation studies, all four were randomized, double-blind, and cross-over by design; three studies used participants 55 years of age and older (Bocca, Le Doze et al. 1999; Meskali, Berthelon et al. 2009; Bocca, Marie et al. 2011), while the mean age in the fourth study was 38 years (Staner, Ertlé et al. 2005). Zopiclone affected driving ability in all four simulations (Bocca, Le Doze et al. 1999; Staner, Ertlé et al. 2005; Meskali, Berthelon et al. 2009; Bocca, Marie et al. 2011); Zolpidem affected driving ability in three simulations (Staner, Ertlé et al. 2005; Meskali, Berthelon et al. 2009; Bocca, Marie et al. 2011), but not in the fourth (Bocca, Le Doze et al. 1999). Zolpidem and Zopiclone were studied individually in three additional studies each. Zolpidem affected driving ability in all three studies (Verster, Volkerts et al. 2002; Partinen, Hirvonen et al. 2003; Otmani, Demazières et al. 2008), while Zopiclone affected driving ability in two of the three studies (Vermeeren, Riedel et al. 2002; Leufkens and Vermeeren 2009; Mets, Vries et al. 2011).

In addition to the eight medications described, four of the other 15 medications associated with increased risk of MVC have not been associated with affected driving ability during driving assessments or simulation studies; this includes Buprenorphine, Methadone, Levocitirizine, and Codeine. One driving simulation study investigated the effects of Buprenorphine and Methadone in a group of former heroin addicts whom were stabilized for at least three months and compared them to a group of healthy controls (Lenné, Dietze et al. 2003). SDLP, speed, steering wheel angle, and reaction time were used to assess driving ability. There was no difference in driving ability between the placebo group and those treated with either Buprenorphine or Methadone (Lenné, Dietze et al. 2003). Another study investigated the 
effects of Levocitirizine through an on-the-road assessment, which measured SDLP and speed to evaluate driving ability (Verster, Weert et al. 2003). No difference in performance was detected among those treated with Levocitirizine and the control group (Verster, Weert et al. 2003). Codeine has been studied in three driving simulations (Linnoila and Hakkinen 1974; Nilsen, Landr et al. 2011; Leung, Chapman et al. 2013). A randomized, double-blind, placebo-controlled, crossover study of 13 healthy volunteers were administered therapeutic levels of Codeine and assessed on SDLP and time to collision under simulation. There were no differences between exposure and control periods (Leung, Chapman et al. 2013). In another simulation study, 70, 16-22 year old professional drivers from the Finnish Army were randomized to various treatment and control groups and assessed on numerous factors during simulation (Linnoila and Hakkinen 1974). The only difference among the Codeine treated group was that they caused slightly more collisions (Linnoila and Hakkinen 1974). In another simulation study, which compared chronic pain users with Codeine, chronic pain users not using Codeine, and placebo group of healthy controls, participants were assessed on reaction time and missed reactions (Nilsen, Landr et al. 2011). No differences were detected in the Codeine treated group (Nilsen, Landr et al. 2011).

While the remaining three medications, Carisoprodol, Dihydrocodeine, and Tramadol, have not been evaluated in driving assessments or driving simulations, their effects on psychomotor performance and/or cognition have been evaluated in laboratory settings. Carisoprodol, a muscle relaxant, can cause users to feel sedated, sluggish, and distracted, especially at high dosages (Zacny, Paice et al. 2011; Zacny, Paice et al. 2012). At therapeutic doses, Carisoprodol users often report feeling no effects of the medication even though their psychomotor functioning is decreased; this situation may cause users of this medication to underestimate its effects and partake in behaviors, such as driving, when they may be impaired (Zacny, Paice et al. 2012). Dihydrocodeine, an opioid prescribed for mild to moderate pain, is known to cause dizziness and mild euphoria especially in new users (Ammon, Hofmann et al. 1999), which may interfere with their ability to drive. Tramadol, an opioid for mild to moderate pain, has been shown to be fairly safe in respect to cognition and psychomotor function, though at higher doses, 
Tramadol has been linked to the worsening of balance (Mintzer, Lanier et al. 2010). It should be noted that poor balance has been linked to higher incidences of MVC (Huisingh, McGwin et al. 2013).

\section{Implications for research}

From these findings, it is apparent that some medications that are associated with an increased risk of MVC do not appear correlated with decreased driving ability. Conversely, while the majority of the medications included in this review were not found to be statistically associated with increased risk of MVC, the inference that these medications are innocuous on one's ability to drive is not advised. There are several plausible reasons why the association between MVC and driving ability were not congruent with all medications presented in this review. First, this incongruence may be attributed to study design. The 27 studies investigating the risk or odds of MVC were observational in nature, whereas the studies investigating driving ability were all randomized control trials (RCT) and therefore, experimental. The studies investigating driving ability were comprised of small sample sizes and were occasionally limited to certain age groups or genders. Therefore, the findings of the RCTs may not be generalizable to the entire population, which is a known limitation of this type of study design (Rothman, Greenland et al. 2008). In addition, the observational studies may not have been adjusted for key confounders whereas this would not have affected the RCTs' results if participants were randomized properly (Rothman, Greenland et al. 2008). Also, most of the RCTs only investigated one or two aspects of driving ability. The act of driving is quite complex in nature. It is possible that other aspects of driving ability may not have been affected by the medication being investigated. It is also plausible that even if driving ability is partially affected by a medication, some drivers may compensate their driving behaviors to avoid a potential collision, which is a known phenomenon (Marottoli, Ostfeld et al. 1993; Martinez 1995; Kelly, Warke et al. 1999). Second, epidemiological research investigating the associations between specific medications and MVC is lacking; this is evident by the number of studies included in this review. Some medications that have been studied via RCTs may not have been investigated observationally in larger populations. Third, many of the RCTs used healthy volunteers. This is problematic as diseasemedication relationships are often difficult to distinguish. It is entirely possible that the disease in which 
the medication is prescribed is affecting an individual's ability to drive or their risk of MVC. It is also possible that driving ability is only affected for a short period of time and that an individual who takes a medication for an extended period of time develops a tolerance. This may not have been detected as the RCTs were often short in duration.

\section{Implications for clinical practice}

The findings of this review have several key clinical implications. First, the association between a specific medication and increased risk of MVC and decreased driving ability is not always clear. There are some specific medications that are most likely driver-impairing as both experimental and observational research has associated them with decreased driving ability and increased risk of MVC, respectively. These include Diazepam, Flunitrazepam, Flurazepam, Lorazepam, Temazepam, Triazolam, Zolpidem, and Zopiclone. For other medications, such as Buprenorphine, Codeine, Methadone, and Levocitirizine, there appears to be an association between the medication and increased risk of MVC, but not driving ability. As for Tramadol, Carisoprodol, and Dihydrocodeine, they appear to be associated with increased risk of MVC, but have not been evaluated in driving assessments or simulations. Secondly, some specific medications belonging to the same category or class may be more detrimental than others. For example, among the "Other Medications" included in this review, Carisoprodol had a strong association with MVC while the other medications in this category did not. Thirdly, some of these medications are widely prescribed. For example, Buprenorphine was the $39^{\text {th }}$ most sold drug in the U.S. during the fourth quarter of 2013 (IMS Health 2014). Therefore, careful consideration of the patient and their lifestyle must be given when these medications are prescribed. Possible alternatives within a drug category or class should be considered for patients that frequently drive as certain medications may be more driver-impairing than others. Education efforts are needed to ensure that patients are aware that certain medications may increase their risk of MVC.

\section{Strengths and limitations of this review}

The strengths of this review are that over 50 years of data among fourteen different databases were searched for potential studies. This review also included relevant 'grey literature' as evidenced by 
the inclusion of three doctoral dissertations and one conference paper. Despite its strengths, this review is not without its limitations. For example, publication bias is a known limitation of systematic reviews (Rothman, Greenland et al. 2008). It is also possible that some studies may have been missed despite two authors independently reviewing studies for inclusion. As much of the DUID research arises from Europe (Kelly, Darke et al. 2004), it is likely that several studies were not included or missed as they were not available in the English language, a requirement of the a priori inclusion criteria. In some instances, only abstracts and not complete studies could be retrieved, especially with reports or conference papers that were dated. The findings of this review also indicate that future research opportunities in this area of study are plentiful. Future research could entail, 1) RCTs of medications found to be associated with increased risk of MVC that were never evaluated in a driving assessment or simulation, 2) epidemiological studies investigating the effects of other medications or new medications that may be driver-impairing, 3) investigating different methods of measuring driving ability in simulations and actual assessments, and 4) pursuing patient or clinician educational efforts to raise awareness about the possible effects of specific medications on MVC or affected driving ability.

\section{Conclusions}

This systematic review sought to determine which specific medications were associated with increased risk of motor vehicle collision among licensed drivers. While the majority of medications investigated were found to not be significantly associated with increased MVC, several medications were associated with such risks. It was also determined that some medications may be more driver-impairing than others. These findings pose numerous clinical implications. Due to the increasing public health and traffic safety concerns regarding driving under the influence of drugs, future research in this area of study is worthy of investigation as the associations between specific medication use and the increased risk of motor vehicle collision or affected driving ability are complex and not always lucid. 


\title{
Chapter 3
}

Licit and illicit drug use among senior drivers killed in U.S. motor vehicle collisions, 2008-2012

\begin{abstract}
Objectives: Adults 65 years of age and older comprise the fastest growing demographic in the U.S. As drug use is projected to increase in this population, there is concern that more seniors will be driving under the influence of impairing drugs. The purpose of this analysis is to explore licit and illicit drug use among senior drivers fatally injured in traffic collisions.

Methods: Data from the Fatality Analysis Reporting System were analyzed from 2008-2012. Commonly used classes and specific drugs were explored. Rates of drug use, multiple drugs, concomitant drug and alcohol use, and alcohol use alone were generated using Poisson regression with robust error variance estimation. Rates for fatally injured senior drivers were compared to those of fatally injured middle-aged drivers (30 to 50 years old) using rate ratios.

Results: Licit and illicit drug use among senior drivers occurred in $17.8 \%$ of those tested. Among drugpositive senior drivers, benzodiazepines and narcotics were frequent. The rates of testing positive for any drug, multiple drugs, combined drug and alcohol, and alcohol use alone among seniors were $47 \%$ $(\mathrm{RR}=0.53,95 \%$ CI $0.50,0.57), 57 \%(\mathrm{RR}=0.43,95 \%$ CI $0.39,0.48), 88 \%(\mathrm{RR}=0.12,95 \%$ CI $0.10,0.15)$ and $79 \%(\mathrm{RR}=0.21,95 \% \mathrm{CI} 0.19,0.24)$ less, respectively, compared to middle-aged drivers.
\end{abstract}

Conclusions: Overall licit and illicit drug use is less common among fatally injured senior drivers relative to middle-aged drivers. As narcotics and benzodiazepines were frequently found in drug-positive senior drivers, driving under the influence of licit drugs may be a growing traffic safety concern. 


\subsection{Introduction}

According to the National Center for Injury Prevention and Control, a person dies nearly every hour in the United States (U.S.) from a motor vehicle collision involving an impaired driver (Centers for Disease Control and Prevention 2015). Despite decades-worth of traffic safety campaigns and nearly 60 billion dollars of annual costs (Centers for Disease Control and Prevention 2015), impaired driving still remains an important public health and traffic safety concern (Kelly, Darke et al. 2004; Walsh, de Gier et al. 2004). While the term 'impairment' traditionally referred to an individual's diminished driving ability resulting from alcohol consumption, impairment also includes driving under the influence of drugs (DUID). Driving under the influence of drugs is not limited to traditional illicit or recreationally used substances, such as cocaine or methamphetamines, which have no real medical benefit. Impairment can be a result of licit drugs, including common prescription and over-the-counter medications, whose effects interfere with one's cognitive, physical, or psychomotor ability to safely operate a motor vehicle from one destination to another (Coopersmith, Korner-Bitensky et al. 1989; Carr 2000; Carr, Duchek et al. 2006; Cheung and McCartt 2011). Between 2007 and 2014, the National Highway Traffic Safety Administration (NHTSA) reports that while alcohol-impaired driving appears to be declining, DUID increased $4 \%$ nationally (National Highway Traffic Safety Administration 2015). Other studies also report increases in the prevalence of prescription drugs among fatally injured drivers (Rudisill, Zhao et al. 2014; Wilson, Stimpson et al. 2014).

While drug-impaired driving has steadily increased in the U.S., so has the number of licensed drivers over 65 years of age. Adults 65 years of age and older (i.e. seniors) comprise the most rapidly growing sub-group of the U.S. population (McGwin, Sims et al. 2000). Should this population trend persist through 2030, 1 in 5 drivers will be 65 or older (Braver and Trempel 2004). Seniors are the largest consumers of both prescription and over-the-counter medications (Bushardt, Massey et al. 2008), and the current generation of older adults reports a higher prevalence of lifetime licit and illicit drug use (Colliver, Compton et al. 2006). Furthermore, it is projected that illicit drug use and non-medical use of prescription drugs will increase in this population through 2020 (Colliver, Compton et al. 2006). 
In spite of these projections, there are numerous gaps in the extant literature. Licit and illicit drug use among drivers, particularly seniors (Lotfipour and Vaca 2007), is largely understudied in the U.S. (Kelly, Darke et al. 2004; Walsh, de Gier et al. 2004). Therefore, the purpose of this analysis is to explore both licit and illicit drug use among drivers 65 years of age or older who were fatally injured in motor vehicle collisions in the U.S. from 2008-2012. While licit substances, such as prescription and over-thecounter medications, can be obtained illegally, misused, or abused, the intent of use is impossible to determine given the decedent nature of the population. The question that this study seeks to answer is, "what are the most common specific and broad groups of licit and/or illicit substances and combinations found in this population of drivers?" Describing the licit and illicit drug use in this demographic may elucidate potential avenues for public health intervention among aging U.S. drivers.

\subsection{Methods}

\section{Data Source}

Data for this analysis were obtained from the Fatal Analysis Reporting System (FARS). FARS is a publicly available database maintained by the NHTSA (National Highway Traffic Safety Administration 2012). All 50 states and the District of Columbia are required to report traffic collisions to the NHTSA when at least one person injured in a traffic collision dies within 30 days of the incident (National Highway Traffic Safety Administration 2012). Trained NHTSA analysts abstract the data from these state reported files using strict quality control procedures (National Highway Traffic Safety Administration 2012). Currently, the FARS database contains over 100 variables relating to the crash, vehicles, and people involved in the incident (National Highway Traffic Safety Administration 2012). In the database, blood alcohol concentration and up to three drug test results can be recorded for each individual involved in a collision. In cases of multiple drug involvement, the possibility exists that drivers can have more than three drugs detected in their system at time of collision, yet only the first three are reported. FARS lists drug test results in the following priority order: 1) narcotics, 2) depressants, 3) stimulants, 4) marijuana, and 5) other drugs (Centers for Disease Control and Prevention 2006). Nicotine, 
aspirin, and/or drugs administered to an individual as part of post-collision medical treatment are excluded from the drug test results (National Highway Traffic Safety Administration 2010; National Highway Traffic Safety Administration 2012). Drug testing can be performed using urine and/or blood. The consistency of drug testing varies greatly by state (National Highway Traffic Safety Administration 2010). Some states routinely test fatally injured drivers for licit and illicit substances, while others do not (National Highway Traffic Safety Administration 2010). Furthermore, states may not consistently report their results to the NHTSA (National Highway Traffic Safety Administration 2010).

\section{Study Population}

Due to the variability in states' drug testing practices and reporting, this analysis was limited to states with acceptable data quality. Each state's data quality was examined from 2008-2012 for a combination of factors set a priori including the overall percentage of fatally injured drivers who were tested, the percentage of population found drug-positive, and the proportion of drug results listed as "Other". For inclusion in this analysis, the state had to test at least $50 \%$ of fatally injured drivers. A state's data quality was considered insufficient if the drug-positive percentage was unusually high (>70\%) or low $(<5 \%)$ and/or the percentage of results described as "Other" was high $(>70 \%)$. The following states $(n=28)$ met the inclusion criteria--Alaska, Arizona, California, Colorado, Florida, Hawaii, Illinois, Indiana, Kentucky, Maryland, Massachusetts, Michigan, Nevada, New Hampshire, New Jersey, New Mexico, New York, North Dakota, Ohio, Pennsylvania, Rhode Island, South Carolina, Vermont, Virginia, Washington, West Virginia, and Wyoming — along with the District of Columbia.

The study population was further limited to fatally injured drivers with a known drug test result from states meeting the inclusion criteria. The traffic collision must have occurred between January 1, 2008 and December 31, 2012. The study population was also limited to those drivers 65 years of age or

older at time of collision. For comparison purposes, middle-aged drivers (i.e. drivers 30-50 years of age at time of collision) were included as the referent population for the analysis. The rationale of using 3050 year olds as the referent group was two-fold: 1) 30-50 year olds are generally safer behind the wheel, 
and 2) they represent the average age of all drivers on the road (Foley, Heimovitz et al. 2002; Cheung and McCartt 2011). The overview of participant selection, along with sample sizes, is described in Figure 1.

\section{Covariates}

Covariates of interest included age group; gender; race; ethnicity; type of drug test administered post-collision; blood alcohol concentration (BAC); a driving while intoxicated (DWI) conviction within the past 3 years; a previous crash within the past 3 years; day; time; and year of the crash; the number of vehicles involved in the collision; weather conditions at time of collision; and how long the individual survived after the crash. With the exception of the type of drug test administered, weather conditions at time of collision, and survival time, all variables were characterized similarly to previously published work (Brady and Li 2013). The type of drug test administered post-collision was categorized as urine, blood, or a combination of urine and blood. Weather condition at time of collision was categorized as adverse (i.e. rain, wind, snow, sleet, hail, fog, freezing rain, or blowing debris) or clear. Survival time was dichotomized into death within one hour of collision or beyond.

BAC, which was measured in grams per deciliter (g/dl), was based on multiple imputed levels dictated by NHTSA if the value was missing for a driver (National Highway Traffic Safety Administration 2012). The NHTSA's multiple imputation method for missing BAC of drivers involved in fatal traffic collisions has been extensively published in the literature (Rubin, Schafer et al. 1998; Subramanian 2002). The NHTSA's validated model imputes 10 specific values of BAC across a range of possible values which allows for the estimation of statistics including measures of central tendency and dispersion (Rubin, Schafer et al. 1998; Subramanian 2002). The overall BAC estimates were generated by combining these 10 specific values via the PROC MIANALYZE procedure using SAS/STAT software (SAS Institute 2010).

\section{Outcome Variables and Definitions}

Several terms pertaining to the outcomes of this analysis, which are presented throughout this paper, include: licit drugs, illicit drugs, any drug use, multiple drug use, drug and alcohol use, alcohol use only, other drugs, broad drug groups, specific/types of drugs, and cannabinoids. The term 'licit drug' is 
defined as prescription and/or over-the-counter medications typically taken to remedy a medical condition. The term 'illicit drug' refers to drugs that are commonly illegal or used recreationally with no real medical benefit; in this analysis, cannabis and cannabis metabolites were considered illicit as they are deemed illegal by federal law. 'Any drug use' is defined as testing positive for at least one licit or illicit drug. 'Multiple drug use' is defined as testing positive for two or more drug categories. 'Drug and alcohol use' is classified as testing positive for at least one licit or illicit drug and having a BAC greater than $0.01 \mathrm{~g} / \mathrm{dl}$. 'Alcohol use only' is characterized as testing negative for illicit and licit drugs but having a BAC greater than $0.01 \mathrm{~g} / \mathrm{dl}$. 'Other drugs' refers to illicit or licit drugs that cannot be categorized as narcotics, depressants, cannabinoids, stimulants, hallucinogens, phencyclidine, anabolic steroids, or inhalants.

'Broad drug categories' are overarching groups of drugs which include narcotics, depressants, cannabinoids, stimulants, hallucinogens, phencyclidine, anabolic steroids, inhalants, and 'Other drugs' 'Specific/types of drugs' are narrower drug classifications which include substances such as benzodiazepines, hydrocodone/oxycodone, barbiturates, cocaine, and methadone. The term 'cannabinoid' refers to cannabis and its metabolites, which include Delta 9, Hashish, Marinol, and Tetrahydrocannabinol.

\section{Statistical analysis}

Descriptive characteristics of senior drivers testing positive for at least one drug were compared to senior drivers testing negative. An additional analysis was conducted to quantify which covariates were associated with drug-positive results in this population of drivers. Drug prevalence rates were assessed for any drug use, multiple drug use, drug and alcohol use, and alcohol use alone in both senior and middle-aged drivers. The drug prevalence rates were calculated by dividing the number of fatalities per drug group by the total number of drivers tested for each age group. The prevalence ratio was then determined by dividing the drug prevalence rates of senior drivers by the drug prevalence rates of middleaged drivers for each drug category or class. Drug prevalence ratios were calculated using hierarchical 
Poisson regression with robust error variance estimation, where random effects were used to account for correlations among state reporting (Zou 2004).

The proportions of select broad drug categories and specific drugs that were identified in both senior and middle-aged drivers who tested positive for licit and illicit drugs were also explored.

Frequencies of the most commonly used specific and broad drug categories were also obtained for both senior and middle-aged drivers that tested positive for licit and/or illicit drugs. These findings were presented for each age group and by the number of drugs detected. The purpose of this analysis was to determine which broad and specific drugs were most often detected in each group of drivers. It should be noted that the a priori level of statistical significance was set at 0.05 and SAS/STAT ${ }^{\circ}$ software (SAS Institute 2010) version 9.3 was used for all analyses.

\section{Sensitivity Analysis}

Due to the variability of reporting among states, a sensitivity analysis was conducted. The purpose of the sensitivity analysis was to determine if the effects of states' overall drug testing percentages biased the results. All analyses previously described were re-run using data from states that routinely tested $80 \%$ or more of their fatality injured drivers. The states $(n=10)$ that met these criteria were California, Hawaii, Maryland, New Hampshire, New Jersey, New Mexico, North Dakota, Vermont, Washington, and West Virginia. To determine if a bias existed, the results from the original analysis and the sensitivity analysis were qualitatively compared.

\subsection{Results}

The descriptive characteristics of the fatally injured senior drivers with complete toxicological testing are presented by drug test status in Table 1 . Of the 5,828 senior drivers included in this analysis, $1,039(17.8 \%)$ were found drug-positive. Among this group of drivers, $28.5 \%$ were between 65-69 years of age, $39.7 \%$ were between $70-79$ years of age, $27.6 \%$ were between $80-89$ years of age, and $4.2 \%$ were 90 years of age or older. The sample consisted mainly of white, non-Hispanic males. The majority of drivers were administered a blood test (91.2\%). Many of the drivers tested were found to have a zero blood alcohol concentration (90.0\%). Of the drivers testing positive for drugs, $62.9 \%$ tested positive for 
only one drug while $23.2 \%$ tested positive for two drugs. Only $14.0 \%$ of those found drug-positive had three or more drugs detected. Over $99 \%$ of the drivers tested had not been recently convicted of a DWI offense and $10.7 \%$ were involved in a recent collision. Among the drivers tested, most collisions occurred between Monday and Thursday between 7:00AM-6:59PM. The collisions typically involved two or more vehicles as opposed to single-vehicle crashes (62.4\% vs. $37.6 \%$, respectively) and occurred when the weather was either clear or cloudy (89.3\%). Approximately $57 \%$ of the drivers tested died within one hour of the collision. Over the 5 year span, the total number of drivers tested declined, but the number of drivers testing positive for drugs increased. The sensitivity analysis showed similar results (data not shown).

Table 2 presents the prevalence and prevalence ratios of drug presence among population subgroups of senior drivers. The purpose of this analysis was to determine which of the demographic characteristics previously described were associated with an increased or decreased occurrence of a drugpositive test. As drivers aged, the prevalence of drug-positive tests decreased. For example, compared to those aged 65-69 years, fatally injured drivers 70-79, 80-89, and $\geq 90$ years of age were approximately $13 \%(\mathrm{PR}=0.87,95 \% \mathrm{CI} 0.76,1.01), 33 \%(\mathrm{PR}=0.67,95 \% \mathrm{CI} 0.57,0.79)$, and $50 \%(\mathrm{PR}=0.50,95 \% \mathrm{CI}$ $0.34,0.75)$, respectively, less likely to have a drug-positive test. There did not appear to be a statistically significant difference concerning gender, race, or ethnicity. Individuals with a BAC of 0.01-0.07 g/dl were $36 \%$ more likely $(\mathrm{PR}=1.36 ; 95 \% \mathrm{CI} 1.01,1.82)$ to have a positive drug result compared to those with a BAC equal to zero. Those that received a previous DWI conviction within the past 3 years tended to test positive for drugs. Individuals testing positive were $16 \%$ more likely (PR=1.16; 95\% CI 1.03, 1.32) to be involved in single vehicle relative to multiple vehicle collisions. The prevalence rate of drugpositive tests steadily increased from 2008-2012. The prevalence rate rose from 135.8 per 1,000 in 2008 to 215.3 per 1,000 individuals testing positive in 2012 . The sensitivity analysis showed similar results (data not shown).

The prevalence rates and ratios of drug involvement among fatally injured senior and middleaged drivers are presented in Table 3. Drug-positive results were $47 \%$ less in senior drivers compared to 
middle-aged drivers $(\mathrm{RR}=0.53 ; 95 \% \mathrm{CI} 0.50,0.57)$. Senior drivers were $57 \%$ less likely to test positive for two or more drug classes ( $\mathrm{RR}=0.43 ; 95 \% \mathrm{CI} 0.39,0.48)$ and $88 \%$ less likely to test positive for drugs and alcohol compared to middle-aged drivers $(\mathrm{RR}=0.12,95 \% \mathrm{CI} 0.10,0.15)$. Fatally injured senior drivers were also $79 \%$ less likely to drink and drive relative to middle-aged drivers $(\mathrm{RR}=0.21 ; 95 \% \mathrm{CI}$ $0.19,0.24)$. The results of the sensitivity analysis were comparable (data not shown).

The proportions of broad drug categories and specific drugs identified in both senior and middleaged drivers that tested positive for licit and/or illicit drugs are presented in Table 4. Among senior drivers who were drug-positive, $43.4 \%$ tested positive for "Other" drugs. Among drug-positive senior drivers, $37.2 \%$ tested positive for narcotics while $31.8 \%$ tested positive for depressants. Testing positive for cannabinoids $(6.4 \%)$ and stimulants $(5.5 \%)$ was considerably less frequent among senior drug-positive drivers. The drug use among middle-aged drivers that tested drug-positive differed from drug-positive senior drivers. Among drug-positive middle-aged drivers, $34.6 \%$ and $30.4 \%$ tested positive for cannabinoids and stimulants, respectively. While slightly less than drug-positive senior drivers, $25.5 \%$ and $23.9 \%$ of middle-aged drug-positive drivers tested positive for narcotics and depressants, respectively. As for specific drugs, $26.1 \%$ of fatally injured drug-positive senior drivers tested positive for benzodiazepines and $17.7 \%$ tested positive for either oxycodone or hydrocodone. Only $1.8 \%$ and $1.4 \%$ of drug-positive senior drivers tested positive for cocaine and methadone, respectively. Among drug-positive middle-aged drivers, $17.4 \%$ tested positive for cocaine, $21.4 \%$ tested positive for benzodiazepines, and $16.4 \%$ tested positive for hydrocodone or oxycodone. The results of the sensitivity analysis were comparable (data not shown).

The most common broad groups of drugs detected in drug-positive drivers are presented in Table 5a by age group and by the number of drugs detected. In regards to drug-positive senior drivers, the majority only had one drug identified post-collision. Among drug-positive senior drivers with one drug detected, "Other" drugs were most commonly detected (42.3\%), followed by narcotics $(25.7 \%)$ and depressants (20.1\%). Among drug-positive senior drivers with two drugs detected, approximately 19\% tested positive for a narcotic and a depressant while $18.3 \%$ tested positive for two narcotics or a narcotic 
combined with an "Other" drug (16.2\%). The drugs detected in fatally injured drug-positive middle-aged drivers differed greatly from drug-positive senior drivers. Among these drug-positive drivers, cannabinoids (37.0\%), stimulants (27.2\%), and "Other" (13.9\%) drugs were the most common broad drug groups, if only one drug was detected. In drug-positive middle-aged drivers with two drugs detected, combinations of a stimulant and cannabinoid (17.6\%) or a narcotic and a depressant $(12.1 \%)$ were most often identified. In the sensitivity analysis, the results of the broad drug categories were virtually identical (data not shown).

The most common specific drugs identified in the drug-positive fatally injured drivers are presented in Table $5 \mathrm{~b}$ by age group and by number of drugs detected. Among senior drivers testing positive for one drug, benzodiazepines [specific type-unknown (7.0\%)], cannabis (6.6\%), hydrocodone (5.4\%), and morphine (2.9\%) were common. Among senior drivers testing positive for two drugs, combinations of two "Other" drugs, followed by combinations of hydrocodone and hydromorphone (4.2\%), hydrocodone and an "Other" drug (4.2\%) and morphine and an "Other" drug (2.9\%) were most common. Conversely, middle-aged drivers testing positive for one drug tended to have cannabis (37.0\%) and cocaine $(14.7 \%)$ most commonly detected. For those with two drugs detected, the most prevalent combinations included cocaine and cannabis (9.4\%), amphetamines and cannabis (8.2\%), and cocaine and an "Other" drug (5.7\%). The results of the sensitivity analysis were comparable (data not shown). Among senior drivers testing positive for one drug, "Other" drugs (41.3\%) followed by cannabis (8.5\%), diazepam (6.5\%), hydrocodone (6.5\%), and morphine (5.5\%) were most common. Among seniors testing positive for two drugs, two "Other" drugs (21.2\%), diazepam and an "Other" drug (3.5\%), and dihydrocodeine and hydrocodone (3.5\%) were the most prevalent combinations. In middle-aged drivers testing positive for one drug, cannabis (35.4\%) and amphetamines (21.5\%) were the most commonly identified drugs. In middle-aged drivers testing positive for two drugs, amphetamine and cannabis $(14.7 \%)$, cocaine and cannabis $(6.3 \%)$ and cocaine and "Other" drugs $(6.3 \%)$ were the most common combinations.

\subsection{Discussion}


The principal finding of this study is that drug use among fatally injured drivers 65 years of age and older was detected in $17.8 \%$ of those tested. Among the drivers testing positive, the majority were confirmed to have only consumed one or two drugs prior to collision. Depressants, such as benzodiazepines, and narcotics, such as hydrocodone, were commonly consumed both separately and in combination. The rates of drug involvement, multiple drug use, drug and alcohol use, and alcohol use alone were considerably less in senior drivers compared to middle-aged drivers. Another interesting finding was that while the number of senior drivers tested gradually decreased over the 5-year period, the prevalence of drug use steadily increased from 2008 to 2012. These findings suggest that while drug use is less frequent among fatally injured senior drivers compared to middle-aged drivers, narcotic and depressant consumption is prevalent among drug-positive senior drivers; there is also evidence that drug use may be increasing in this population.

These findings are consistent with the current literature. It is estimated that approximately $60 \%$ of all adults over 65 years of age consume one prescription medication daily (Fulton and Allen 2005), while only $20 \%$ of seniors use three or more prescriptions each day (Jorgensen, Johansson et al. 2001). In this analysis, the majority of senior drivers were found negative for both licit and illicit drugs, which was surprising considering that prescription drug use generally increases with age. There may be several potential explanations for this result. First, previous research demonstrates that drivers self-regulate and alter their traffic behaviors when they feel that their driving ability is compromised due to poor health (Marottoli, Ostfeld et al. 1993; Martinez 1995; Kelly, Warke et al. 1999). Such drivers will typically avoid situations involving traffic congestion, complex intersections, adverse weather, driving alone, and/or driving at night (Ball, Owsley et al. 1998; Blanchard, Myers et al. 2010). Because the participants in this study were still driving, it is possible that the drivers included in this analysis were healthier and consequently consuming fewer medications than their less healthy counterparts. An alternative explanation may involve gender differences. Through the aging process, women typically drive less and/or cease driving sooner than men (Stewart, Moore et al. 1993). Research also suggests that women typically consume more medications and have higher rates of multiple medication use compared to males 
(Jorgensen, Johansson et al. 2001). As the study population was comprised predominately of men, the rates of medication use may have naturally been lower than if it were equally comprised of males and females. A third, though unlikely, explanation for the number of negative drug tests, may have been the timing of the collisions and/or medication adherence. The majority of the collisions occurred on weekdays during daylight hours. It is possible that some drivers had prescriptions but did not yet take their medications or were non-adherent to their medication regimen on the day of their collision. While age and gender are not believed to be predictors of medication adherence, the number of prescriptions are; medication adherence varies inversely with the number of prescriptions an individual takes each day (Hughes 2004). Hence, if an individual has multiple prescriptions, their medication adherence may be low.

The high prevalence of negative drug test results for illicit substances and alcohol is also consistent with the current literature. Previous research suggests that illicit drug and alcohol use may not be commonplace among senior drivers. A study conducted during the 1990's in Tennessee revealed that only $14 \%$ of Level 1 trauma patients over 60 years of age whom were involved in motor vehicle collisions tested positive for alcohol over the five year study period. In this same study, less than $1 \%$ of these patients tested positive for illicit drugs (Higgins, Wright et al. 1996).

While alcohol and illicit drug use appears atypical among senior drivers, the high prevalence of narcotics, depressant, and "Other" drugs use among fatally injured senior drivers is not unprecedented given the following recent trends: 1) increased retail drug sales, 2) elevated emergency department visits, 3) increased hospital admissions, and 4) high death rates due to these substances. Among all written prescriptions, cardiovascular, central nervous system, and gastrointestinal medications are typically the most prevalent pharmacologic agents prescribed to elderly individuals (Jorgensen, Johansson et al. 2001). It is possible that the medications listed as "Other" were these types of pharmacological agents; it is also possible that these substances were over-the-counter medications as elderly adults are the largest consumer of such products based on retail drug sales (Fulton and Allen 2005). Unfortunately, due to the constraints of the FARS database, discerning what type of pharmacologic agent the "Other" drugs were 
remains unknown. As for the high prevalence of opioid analgesics and benzodiazepines among fatally injured senior drivers, their use in the U.S. has dramatically increased over the past two decades (Joranson, Ryan et al. 2000; Paulozzi, Ballesteros et al. 2006). The retail sales of oxycodone and hydrocodone rose almost 600\% and 200\%, respectively, between 1997 and 2005 (Manchikanti 2007). The number of emergency department visits due to opioid and benzodiazepine use increased $111 \%$ and 89\%, respectively, between 2004 and 2008 (Centers for Disease Control and Prevention 2010), which may be partially attributed to the concomitant use of these medications (Jones, Mogali et al. 2012). Hospitalizations due to opioids and benzodiazepines increased $400 \%$ and $39 \%$, respectively, between 1999 and 2006 (Coben, Davis et al. 2010), while death rates attributed to opioid analgesics was almost four times higher in 2008 compared to 1999 (Centers for Disease Control and Prevention 2011). These trends may be attributed to several factors such as the rise and popularity of internet pharmacies (Manchikanti 2007), increases in generic drug manufacturing (Adis International 2002; Bailey, Barton et al. 2006; Srihari, Padmaja et al. 2009), and national initiatives to address the under-treatment of pain which have been associated with increases in opioid prescriptions (Manchikanti 2007; Pletcher, Kertesz et al. 2008).

While licit drugs were detected in a portion of this population, the findings of this analysis are not suggesting causation. The fundamental challenge of studying the association between licit drugs and driving ability is that the relationship is not as clear as compared to alcohol (National Highway Traffic Safety Administration 2010) for numerous reasons. First, drugs may affect individuals differently. This could be attributed to a myriad of factors such as an individual's genetics (Daly 2014), current health status (Bushardt, Massey et al. 2008), drug dosaging (Brown, Milavetz et al. 2013), drug interactions (Bushardt, Massey et al. 2008), or developed tolerance (Stein and Baerwald 2014). Secondly, not all drugs affect driving ability. Although, several drugs, including some of those found in this analysis, have been linked to increased motor vehicle collisions including barbiturates (Christensen, Nielsen et al. 1990), insulin (Hours, Fort et al. 2008), antihistamines (Verster and Volkerts 2004), narcotics (Bachs, Engeland et al. 2009), antipsychotics (Carr 2000), and muscle relaxants (Carr 2000). Third, disease-medication 
relationships are nearly impossible to distinguish. It is possible that disease may be affecting an individual's driving ability and not the medication taken to mitigate symptoms or disease progression. Several medical conditions have been associated with increased risk of motor vehicle collision including sleep apnea (Ellen, Marshall et al. 2006), dementia (Brown and Ott 2004), arthritis (Cross, McGwin et al. 2009), diabetes (Hansotia and Broste 1991), epilepsy (Hansotia and Broste 1991), anxiety (Sagberg 2006), depression (Sagberg 2006), and Parkinson's disease (Uc, Rizzo et al. 2006). Given the decedent nature of the population, these points could not be explored.

\section{Limitations}

The strengths of this analysis are the use of multi-state data over a 5-year period. The weaknesses of this study are the limitations imposed by the FARS data. As previously mentioned, FARS does not list every drug in existence and many substances are listed as "Other". Therefore, it is unknown what type of pharmacologic agent (i.e. over-the-counter, prescription, etc.) an "Other" drug was. Future research will likely involve trying to discern what other types of specific medications and combinations senior drivers involved in motor vehicle collisions consume. A second limitation of the FARS data involves metabolites. FARS simply lists the drugs detected in individuals; some of these reportable substances may be metabolites. It may be difficult to determine the parent drug an individual consumed based on the metabolite reported, particularly if the parent drug was not detected or if a certain pharmacologic agent is a metabolite of more than one drug. A third limitation of the FARS data is that not all drivers killed in fatal crashes are tested for drugs, and differences in state reporting do exist. The sensitivity analysis sought to address these issues. Additionally, a maximum of three drugs can be reported per person in FARS, which could be a bias in instances of extreme multiple-drug involvement. Furthermore, the results of this analysis also do not prove that the drugs identified were the cause for collision or that —in the case of legal, prescription drugs - they were being misused or abused by the driver. Also, the study population included more males than females; future studies could investigate gender differences in licit and illicit drug use among drivers injured or killed in motor vehicle collisions.

\section{Conclusions}


While the rates of licit and illicit drug use were considerably less among fatally injured senior drivers compared to fatally injured middle-aged drivers, some individuals in this population consumed medications which may have impaired their driving ability. Among drug-positive senior drivers killed in U.S. traffic collisions, narcotic analgesics and benzodiazepines were often detected separately or in combination. These findings suggest that driving under the influence of prescription medication may be a growing public health and traffic safety concern for the aging U.S. population. Discernment must be raised amongst the public and healthcare providers that driving under the influence of commonly prescribed medications may be perilous not only to drivers, but to their passengers, pedestrians, and others frequenting U.S. roadways. 


\title{
Chapter 4 \\ Medication use and the risk of motor vehicle collision in West Virginia drivers 65 years of age and older: a case-crossover study
}

\begin{abstract}
Background: The current generation of older adults reports a higher lifetime prevalence of prescription, over-the-counter and recreational drug usage. As older adults continue to drive later in life, concerns regarding how specific drugs affect driving ability are beginning to accrue. The purpose of this analysis is to characterize the drug usage and determine the risk of motor vehicle collision associated with individual medications in a population of drivers $\geq 65$ years of age.
\end{abstract}

Methods: A case-crossover study was conducted at West Virginia University Healthcare's facilities using data obtained from the electronic health records $(n=611)$ of drivers aged $\geq 65$ years who were admitted for treatment following a motor vehicle collision which occurred between Jan. 1, 2009 and June 30, 2014. Medication usage 14 days before the collision was matched to the medication usage which occurred during four control periods 350-365 days, 255-270 days, 165-180 days, and 75-90 days before the crash using conditional logistic regression.

Results: Analgesic, cardiovascular and gastrointestinal medicines were prevalent. Few drivers tested positive for drugs. Of those testing positive, benzodiazepines and opiates were the most common. After adjusting for the number of medications taken during case and control periods, drivers consuming Tramadol (OR: 11.41; 95\% CI 1.27, 102.15) were at a significantly increased risk of motor vehicle collision while taking this medication 14 days prior to collision compared to control periods.

Conclusions: Older adult drivers who have a prescription for this medication should be made aware of its potential risks. Further research is necessary in a larger, more nationally representative population as many other drugs were trending toward being associated with motor vehicle collision though were not found statistically significant due to small sample sizes and/or low statistical power. 


\subsection{Introduction}

The United States (U.S.) is experiencing an unprecedented demographic shift as the number of adults 65 years of age and older (i.e. seniors) are the most rapidly growing subgroup of the population (McGwin, Sims et al. 2000). By 2020, it is estimated that the number of licensed drivers over 65 years of age will exceed 40 million (Awadzi, Classen et al. 2008). This demographic shift poses a unique challenge to both public health and traffic safety officials as senior drivers experience more motor vehicle fatalities (Massie, Campbell et al. 1995) and an increased rate of injurious crashes per mile driven compared to any other age group (Keall and Frith 2004).

It is also well-established in the literature that medication usage increases with age; in the U.S., adults over 65 years of age consume more than $30 \%$ of all annual written prescriptions (Bushardt, Massey et al. 2008). The current generation of older adults reports a higher prevalence of both lifetime licit (i.e. prescription and over-the-counter medications used to treat a medical condition) and illicit drug use (i.e. drugs used recreationally with no real medical benefit) compared to previous generations (Colliver, Compton et al. 2006). As more adults continue to drive later in life compared to previous generations, concerns regarding how these drugs affect driving ability are beginning to amass (Kelly, Darke et al. 2004; Walsh, de Gier et al. 2004). While alcohol is a known contributor of motor vehicle collisions, the extent to which drugs other than alcohol contribute to crashes is less lucid.(Centers for Disease Control and Prevention 2006) There is evidence that driving under the influence of drugs is increasing nationally and that prescription medication, some of which may interfere with central nervous system functioning, are becoming more ubiquitous than illicit drugs among fatality injured drivers (Rudisill, Zhao et al. 2014; Wilson, Stimpson et al. 2014).

The association between medications and motor vehicle collisions is largely understudied in the U.S. (Kelly, Darke et al. 2004; Walsh, de Gier et al. 2004), particularly among senior drivers (Lotfipour and Vaca 2007). This issue may be of particular importance to West Virginia residents. The population 
of West Virginia is more mature compared to other states with more than $16 \%$ of the state's residents being over 65 years of age (West Virginia Department of Health Bureau of Family Planning 2010). Previous research has also shown that both licit and illicit drug use contribute greatly to motor vehicle collisions in West Virginia (Centers for Disease Control and Prevention 2006). The traffic fatality rate is also starkly higher-nearly $45 \%$ more--compared to other non-Appalachian states (Zhu, Zhao et al. 2013). Therefore, the purpose of this analysis is to explore which prescription and over-the-counter medications are most common, if illicit drug use is prevalent, and which prescription and over-the-counter medications are associated with an increased risk of motor vehicle collision among West Virginia drivers 65 years of age or older. Discerning this information may help guide future interventional and educational efforts to minimize death and disability from motor vehicle collisions in this population.

\subsection{Methods}

\section{Study Design}

The design for this analysis is a case-crossover. Developed by Maclure in 1991, case-crossover studies are similar to matched case-control studies in theory; the fundamental difference between the two study designs is that cases serve as their own controls in a case-crossover study (Maclure 1991). The case-crossover design compares (i.e. matches) a case's exposure during a time period immediately preceding an event (i.e. the case or 'risk' period) to the exposure in a time period when the event did not occur (i.e. control period) (Maclure 1991). These designs are useful for studying the relationship between transient exposures and acute outcomes; they are commonly used in air pollution studies, traffic safety, and pharmacoepidemiology (Wang, Schneeweiss et al. 2004). A benefit of the case-crossover design is that fixed confounders, such as age, race, sex, etc, are controlled for regardless if they were actually measured (Greenland 1996). The sampling schema for this analysis is depicted in Figure 1. The event of interest is a motor vehicle collision in which the driver required medical treatment. The exposure of interest is the driver's medication usage leading up to the collision. The risk period is defined as the 14 
days preceding the motor vehicle collision. The reason as to why 14 days was chosen as the risk period as opposed to one of shorter duration is because medications may take time to accumulate and/or cause side-effects in an individual which may interfere with their driving ability. Additionally, this length of risk period has been used in previous studies (Barbone, McMahon et al. 1998). In order to increase statistical efficiency (Mittleman, Maclure et al. 1995), there are four matched control periods, each 15 days long, at 350-365 days, 255-270 days, 165-180 days, and 75-90 days before the collision. Control periods were chosen to be disjoint from one another to avoid possible correlation (Lumley and Levy 2000).

\section{Study setting}

All cases received medical treatment from West Virginia University (WVU) Healthcare's facilities located in Morgantown, WV. Morgantown is a city with approximately 29,600 residents situated in Monongalia County in north-central West Virginia (West Virginia Department of Health and Human Resources 2010). The size of the town can fluctuate throughout the year as it is home of West Virginia University, the state's largest institution of higher education. In 2012, approximately $25.1 \%$ of the patients served by WVU Healthcare resided in Monongalia county, while an additional $21.5 \%$ of patients lived in surrounding counties (i.e. Preston, Marion, and Taylor counties) (West Virginia University Healthcare 2013). Approximately $38 \%$ of patients who sought treatment from WVU Healthcare were from other West Virginia counties, while another $\sim 15 \%$ of patients were from surrounding states (i.e. Pennsylvania, Maryland, Ohio, Virginia and Kentucky) (West Virginia University Healthcare 2013). Therefore, the population served by WVU Healthcare is fairly representative of the state. The population of West Virginia is predominately of white non-Hispanic race/ethnicity and is comprised largely of older individuals (West Virginia Department of Health and Human Resources 2010). In 2010, heart disease, cancer, and chronic lower respiratory disease were the leading causes of death in this population (West Virginia Department of Health and Human Resources 2010). 


\section{Data sources and collection}

All data for this analysis were collected from the electronic medical records of eligible cases and obtained through Medsite and Epic (Merlin), WVU Healthcare's electronic medical record systems. Data was collected through a combination of manual data abstraction and through the assistance of the West Virginia University Clinical and Translational Science Institute's (WVCTSI) Integrated Data Repository. In order to ensure the congruence of the data obtained from WVCTSI and the actual medical records, several electronic records were pulled and compared to ensure accuracy. This study was approved by West Virginia University’s Institutional Review Board (protocol \#1401165743).

\section{Case selection}

The sampling frame consisted of individuals 65 years of age and older at time of treatment and received treatment from emergency or trauma services or urgent care facilities from January 1, 2009 to June 30, 2014. Cases had to be designated as a driver involved in a motor vehicle collision by the International Classification of Disease, Ninth Revision, Clinical Modification codes (ICD 9-CM) E810.0, E811.0, E812.0, E813.0, E814.0, E815.0, E816.0 and E819.0. The E-codes used to identify the incident cases could have been listed as the primary or secondary diagnosis in the electronic medical record.

\section{Medication exposures}

Medications of interest were those considered potentially driver-impairing, meaning that they could possibly alter a driver's cognition, psychomotor function, or physical functioning as suggested by the American Medical Association (American Medical Association 2010). Medication usage up to a year before each case's admittance for treatment was assessed. Medications that were started up to 24 hours before admittance for treatment were not included in the analysis; this was done to avoid potential bias in the event that a medication was administered to a patient by emergency medical services (i.e. the medication was consumed after the collision occurred). The duration of time that an individual was taking a particular medication was obtained directly from the medical record. If the duration of 
medication usage could not be determined or was not documented in the record, the medication was not included in the analysis as to avoid misclassification. As many of the cases were existing patients of WVU Healthcare and/or due to the severity of the incurred injuries, medication records were fair in terms of completeness. Of the 611 case visits included in this analysis, 581 cases had medications noted in their electronic health record at any point, while 292 cases had medication usage in the year preceding their motor vehicle collision. An individual was considered 'exposed' if they were taking a medication at any point during the case and/or individual control periods.

While the purpose of this analysis was to explore the risk of motor vehicle collision due to individual medication exposures, an analysis of more encompassing pharmaceutical sub-classes was also conducted. This included anticholesteremics, anticoagulants, antidepressants (non-benzodiazepines), antihyperglycemics, antihypertensives, narcotic analgesics, and benzodiazepines. In this particular analysis, anticholesteremics included 3-hydroxy-3-methyl-glutaryl-CoA reductase inhibitors.

Anticoagulants included both coumarin type and platelet aggregation inhibitors. Antidepressants included tricyclic antidepressants, selective serotonin re-uptake inhibitors, serotonin antagonist and reuptake inhibitors, serotonin-norepinephrine reuptake inhibitors, norepinephrine-dopamine reuptake inhibitors, and noradrenergic and specific serotonergic antidepressants. Antihyperglycemics included insulin, biguanides, insulin-release stimulants, combination biguanides and insulin-release stimulants, and thiazolidinediones. Antihypertensives included angiotensin-converting-enzyme inhibitors, angiotensin receptor antagonists, and sympatholytic vasodilators. Narcotic analgesics also included narcoticnonsalicylate combinations, and anesthetic adjunct agents.

\section{Other covariates}

Other variables of interest included age, gender, race, day, time, year, and season of admittance, how the patient was transported to medical treatment, treatment location, injury severity, insurance status of the patient, county and state of residence, employment status, length of hospital stay, health status, and 
whether the patient was tested for drugs and/or alcohol at time of admittance. Age was categorized as 65$69,79-79,80-89$ and $\geq 90$ years. Due to the homogeneity of the population, race was classified as white or other. Day of admittance was categorized as weekday (i.e. Monday thru Thursday) or weekend (i.e. Friday thru Sunday). Time of admittance was classified as daytime (7:00 AM to 6:59 PM) or night (7:00 PM-6:59 AM). For season of admittance, winter included December, January, and February, while spring comprised March, April, and May. Summer included June, July, and August while fall included September, October, and November. For method of transport, this was categorized as ambulance, helicopter, or self-transport. Treatment location was grouped into trauma, emergency or urgent care departments; because so few patients were seen by urgent care (i.e. less than 10), urgent care and emergency were reported together to protect patient confidentiality. Patient's injury severity was based on acuity level at time of arrival for treatment. Patient acuity levels that were noted as emergent or immediate were classified as severe injuries. Acuity levels that were urgent were classified as moderate injuries. Acuities that were less urgent or non-urgent were classified as minor injury severity. Since acuity is not assigned within the Urgent Care facility, these were left unassigned. Insurance status of the patient was grouped as government, private, none, or other/unknown. Government insurance included Medicare or Medicaid programs. Private insurance indicated that a patient had a commercial insurance plan. If patient's self paid, insurance status was noted as none. Because there were few other types of insurance, these were grouped with unknown. Because many patients resided in West Virginia, particularly Monongalia County, county and state of residence were grouped as Monongalia or other and West Virginia or other, respectively, to protect patient confidentiality. Employment/work status was categorized as retired or employed. If a patient reported that they were still working full, part-time or were self-employed, employment status was categorized as employed. Chronic disease status was categorized as $0,1-3$, or 4 or more based on the number of chronic conditions patients were noted to have in their medical records. For patient confidentiality purposes, these conditions were grouped as opposed to listing each individually. The chronic conditions of interest, along with Clinical Classification Software codes or ICD 9-CM codes used to identify them in patients' records, were as follows: heart 
disease $(96,97,100,101,103-108,114,117)$, stroke $(109,111,112,113)$, dementia (653), Alzheimer's disease (331.0), diabetes (49,50), cancer (11-44), arthritis (201-203), Parkinson's disease (79), hypertension (98-99), asthma (128), chronic obstructive pulmonary disease (127), alcoholism (660), depression $(296.2,296.3,311)$, anxiety (651), chronic kidney disease (158), and substance abuse/dependency (661). As part of the admission process, patient's urine may have been laboratory tested for the presence of alcohol and/or drugs. The drugs that can be detected are amphetamines, barbiturates, benzodiazepines, buprenorphine, cannabis, cocaine, opiates, phencyclidine and propoxyphene. Results of drug testing were also noted.

\section{Statistical methods}

Because of the 1:4 matching of case to control periods, conditional logistic regression was used to calculate the odds of motor vehicle collision for each medication exposure (Greenland 1999). Because of the study design (i.e. known longitudinal medication exposure over time), the odds ratio approximated the incidence rate ratio (i.e. risk) (Rothman, Greenland et al. 2008). To account for the slight difference in exposure lengths of case and control periods (i.e. 14 days versus 15 days, respectively), the natural $\log$ of exposure time in days was used as the variable offset as suggested by Greenland (Greenland 1999). Because case-crossover studies are not immune to within person confounding, all regression models were adjusted for the number of medications a case was taking during each risk and control period. The number of medications used served as a proxy of health status as a case's health may have been timevarying (i.e. improved or declined) over the study period. All analyses were run using SAS/STAT Software version 9.3 (SAS Institute 2010), with $\alpha=0.05$.

\subsection{Results}

The characteristics of cases and the circumstances surrounding their medical visits ( $\mathrm{n}=611)$ are presented in Table 1. The majority of cases were aged 65-69 (36.8\%) or 70-79 years $(41.2 \%)$ at time of treatment. More males $(54.0 \%)$ than females $(46.0 \%)$ were admitted for treatment post-collision and 
most were of white race (97.0\%). Over $60 \%$ of the medical visits occurred during the week and during daylight hours (75.7\%). More collisions occurred during the spring time (30.9\%) and more patients were admitted during $2012(25.2 \%)$ as opposed to other years. While the method of transport to medical services was unknown for a large portion of the population (43.2\%), ambulance was the primary method of transfer among those with known transportation. As less than 10 patients were seen at urgent care, most patients were treated in the emergency department as opposed to trauma service $(22.1 \%)$, though many of the injuries sustained were moderate to severe. Most patients had government $(54.2 \%)$ or private $(29.6 \%)$ insurance coverage. The majority of patients were West Virginia residents $(80.4 \%)$. While most patients were no longer working, $12.4 \%$ still held some form of employment. Chronic conditions (i.e. 1 or more) were common (82.4\%). Only $32 \%$ of patients were tested for drugs at time of admittance, while slightly more $(44 \%)$ were tested for alcohol.

Table 2 elaborates on the findings associated with the drug testing of cases. Among those tested for drugs at time of admittance, $17 \%$ tested positive for benzodiazepines and $15.5 \%$ tested positive for opiates. Of those that tested positive for benzodiazepines and opiates, nearly $50 \%$ had a traceable prescription for the substance during the past year. It is possible that the other $50 \%$ received a prescription for these substances outside of the WVU Healthcare network, consumed pills left over from older prescriptions, possibly from sharing medications, or simply had incomplete documentation in their medical record. Overall, drug use was not common in those tested as drug tests were negative for amphetamines, barbiturates, buprenorphine, cocaine, and phencyclidine.

Table 3 lists the most common broad therapeutic groups of medications, individual medications, and combination drugs found in the population. Analgesic, cardiovascular and gastrointestinal medications were the most prominent therapeutic groups. As for specific medications, Aspirin, Metoprolol, Lisinopril, and Furosemide were the most common. A combination of Oxycodone and Acetaminophen was the most common dual-drug compound. 
Table 4 lists the most prevalent medications by therapeutic class along with subsequent unadjusted and adjusted odds ratios and $95 \%$ confidence intervals (CI) to approximate the risk of an individual's involvement in a motor vehicle collision. After adjusting for the number of medications a driver was taking during each case and control period, individuals who were taking Tramadol $(\mathrm{OR}=11.41$; $95 \%$ CI 1.27, 102.15) were at a significantly increased risk of motor vehicle collision while taking this substance during the risk period compared to control periods. Though not statistically significant, those taking Clopidogrel, Gabapentin, Citalopram, Insulin, Hydrochlorothiazide, Metoprolol, Zolpidem, and Nitroglycerine were trending towards an increased risk of collision.

Table 5 lists larger therapeutic classes of medications along with subsequent crude and adjusted odds ratios and 95\% CI to approximate the risk of an individual's involvement in a motor vehicle collision while taking these substances during the risk period compared to control periods. After adjusting for the number of medications a driver was taking during each case and control period, all therapeutic classes were found statistically insignificant. Although those taking anticoagulants, antihyperglycemics, and antihypertensive medications during case periods were trending towards an increased risk of motor vehicle collision compared to control periods.

\subsection{Discussion}

This analysis sought to explore which licit drugs were most common, if illicit drug use was prevalent, and which licit drugs were associated with an increased risk of motor vehicle collision among drivers 65 years of age or older who received medical services at WVU Healthcare facilities post-motor vehicle collision using a case-crossover approach. Two principal findings were generated as a result of this analysis. First, while few patients were tested for drugs at time of medical treatment, the drivers found drug-positive tended to test positive for prescription medications. Typical illegal drugs, such as cocaine or phencyclidine, were not common in this population. Second, despite small sample sizes, those taking Tramadol were at a significantly increased risk of motor vehicle collision if they took this 
substance 14 days prior to collision compared to other control times. Numerous other medications were also trending towards an increased risk of motor vehicle collision but were likely not found statistically significant due to small sample sizes and low statistical power. Consequently, these findings may be of important clinical relevance and worthy of further exploration in a larger population.

The findings from this analysis are consistent with the current literature. Trend analyses have shown that prescription medications, particularly benzodiazepines and narcotic analgesics, are being detected more frequently than traditional illicit substances, such as cocaine and methamphetamine, among drug-positive drivers in the U.S. (Rudisill, Zhao et al. 2014; Wilson, Stimpson et al. 2014). While the literature regarding drug usage among older adult drivers is limited, previous research has suggested that illicit drug usage may not be common among this population. A study of drug usage among level 1 trauma patients over 60 years of age involved in motor vehicle collisions $(n=180)$ was conducted by Vanderbilt University (Higgins, Wright et al. 1996). Their analysis revealed that alcohol and illicit drugs were detected in only 14\% and 1\%, respectively, of their study participants (Higgins, Wright et al. 1996). Therefore, the findings from this analysis were similar.

As for the risk of motor vehicle collision posed by individual medications, two previous studies have investigated the risk of motor vehicle collision associated with the use of Tramadol (Bachs, Engeland et al. 2009; Gibson, Hubbard et al. 2009). In the study by Bachs et al, Tramadol use was not significantly associated with an increased risk of motor vehicle collision among a Norwegian cohort of adult drivers aged 18-70 years, though it was trending in the direction of increased risk (Risk: 1.5; 95\% CI $0.9,2.3)$. In the study by Gibson et al, Tramadol was significantly associated with an increased risk of collision (Risk: 9.17; 95\% CI 7.81, 10.77) in an English cohort of drivers aged18-74 years. At high doses, Tramadol is also known to affect balance (Mintzer, Lanier et al. 2010). Poor balance has also been linked to an increased risk of motor vehicle collision, particularly in older populations (Gustavsen, Bramness et al. 2008). Therefore what this analysis adds to the extant literature is that the effects of 
Tramadol are likely not age-dependent because this analysis was limited to older adult drivers whereas other studies investigated drivers less than 74 years of age and the results were comparable.

Numerous other medications were also trending towards an increased risk of motor vehicle collision but were likely not found statistically significant due to small sample sizes and low statistical power. This included Clopidogrel, Gabapentin, Citalopram, Insulin, Hydrochlorothiazide, Metoprolol, Zolpidem, and Nitroglycerin. To the authors' knowledge, no studies have investigated whether Clopidogrel affects driving ability. Two studies have investigated the relationship between anticoagulants and the risk/odds of motor vehicle collision. A case-control study conducted by McGwin et al showed that elderly drivers in Alabama during 1996 who consumed anticoagulants were 2.6 times more likely to be involved in a motor vehicle collision (OR 2.6; $95 \%$ CI 1.0, 6.7) (McGwin, Sims et al. 2000). As to which medications were included in this categorization was not described. A case-control study conducted by Delaney et al in Quebec, Canada showed that those taking Warfarin, another anticoagulant, were not at an increased risk of motor vehicle collision (OR 0.74; $95 \%$ confidence interval $0.55,1.05$ ) (Delaney, Opatrny et al. 2006), which was similar to what was seen in this analysis. Gabapentin, a newer anti-epileptic medication, is not known to affect driving ability, though it may. In a study conducted by Martin et al, healthy senior adults (mean age 66.5 years) experienced mild cognitive effects during psychomotor testing conducted in a laboratory setting (Martin, Meador et al. 2001). Common complaints regarding Gabapentin use include dizziness, nausea, and somnolence (Gupta and Li 2013), which could affect one's ability to drive. While Citalopram has not been associated with an increased risk of motor vehicle collision in two other studies (Gibson and Hubbard 2009; Coupland and Dhiman 2011), secondgeneration antidepressants are known to effect driving ability possibly due to side effects produced after initial use (Chang, Wu et al. 2013). It is possible that those taking Citalopram in this analysis were experiencing side-effects, though it is unknown. Several studies have investigated the association between Insulin use and the risk of motor vehicle collision (McGwin, Sims et al. 2000; Sims, McGwin et al. 2000; Cui 2001; Hemmelgarn, Lévesque et al. 2006; Lonnen, Powell et al. 2008; Skurtveit, Strøm et 
al. 2009). Most of the findings concerning Insulin use are mixed. Two studies have found significantly increased risks of motor vehicle collision with Insulin use (Cui 2001; Skurtveit, Strøm et al. 2009), while four others have found no significant associations (McGwin, Sims et al. 2000; Sims, McGwin et al. 2000; Hemmelgarn, Lévesque et al. 2006; Lonnen, Powell et al. 2008). To the authors' knowledge, no studies have investigated the risk of motor vehicle collision associated with individual antihypertensive medications. One study by $\mathrm{McGwin}$ et al did investigate the use of angiotensin-converting enzyme (ACE) inhibitors, beta-blockers, and diuretics, which all can be used to treat hypertension (McGwin, Sims et al. 2000). After adjusting for age, sex, race, and annual miles driven, the odds of motor vehicle collision were 1.6 (95\% CI: 1.0, 2.7), 1.4 (95\% CI: 0.8, 2.3), and 0.9 (95\% CI: 0.5, 1.7) for ACE inhibitors, beta blockers, and diuretics, respectively (McGwin, Sims et al. 2000). In this analysis, Hydrochlorothiazide, a diuretic, and Metoprolol, a beta blocker, were both trending toward being associated with an increased risk of motor vehicle collision. While the pharmacokinetic properties of these drugs may be a potential explanation, the reason as to why the effects of Hydrochlorothiazide on motor vehicle collision were so pronounced is unknown. Zolpidem, a sleep-promoting medication, has consistently shown in four other studies to be associated with an increased risk of motor vehicle collision (Gustavsen, Bramness et al. 2008; Orriols, Philip et al. 2011; Yang, Lai et al. 2011; Hansen 2012). While no studies have investigated the effects of individual vasodilators, such as Nitroglycerin, on motor vheicle colllsion, only one study investigated them as a pharmaceutical sub-class. Mcgwin et al found that vasodilators were not assocated with an increased risk of collision (Mcgwin, Sims et al. 2000). The reason as to why Nitroglycerin use in this analysis was trending towards an increased risk of motor vehicle collsion remains unknown.

The findings from this analysis have several key clinical implications. First, this study found Tramadol to be associated with an increased risk of collision and numerous other substances such as Clopidogrel, Gabapentin, Citalopram, Insulin, Hydrochlorothiazide, Metoprolol, Zolpidem, and Nitroglycerin, use to be trending towards an increased risk of motor vehicle collision. While these results 
are not conclusive, it may be worthy of notifying older patients who have prescriptions for these medications of their potential risk, particularly if they drive frequently. Future interventional efforts could involve raising patient awareness. Secondly, the findings of this study may suggest that drugs within the same pharmaceutical class maybe more or less driver impairing than others. This may be worthy of consideration to clinicians when prescribing medications to patients who drive frequently.

The findings from this analysis do need to be interpreted with caution as disease-medication relationships are often difficult, if not impossible, to distinguish. It is entirely possible that the disease in which the medications were prescribed could be affecting one's ability to drive or their risk of motor vehicle collision. Numerous medical conditions have been associated with motor vehicle collision, particularly sleep apnea (Ellen, Marshall et al. 2006), dementia (Brown and Ott 2004), arthritis (Cross, McGwin et al. 2009), diabetes (Hansotia and Broste 1991), epilepsy (Hansotia and Broste 1991), anxiety (Sagberg 2006), depression (Sagberg 2006), and Parkinson's disease (Uc, Rizzo et al. 2006). In addition, drugs that affect the central nervous system may exhibit different effects among individuals. There are numerous intrinsic and extrinsic factors that can alter medication effectiveness and/or side effects among individuals. These include, but are not limited to, drug solubility (Augustijns, Wuyts et al. 2014), intestinal pH (Augustijns, Wuyts et al. 2014), drug interactions (Berta, Harangi et al. 2014), age (Brzaković, Vezmar Kovačević et al. 2012), sex (Brzaković, Vezmar Kovačević et al. 2012; Chu 2014), weight (Brzaković, Vezmar Kovačević et al. 2012) ,diet (Siegmund, Siegert et al. 2012; Cingi, Toros et al. 2013), genetics (Daly 2014), circadian rhythms (Innominato, Roche et al. 2014), supplement use (Markert, Ngui et al. 2014), health of the individual (Joy, Frye et al. 2014), developed tolerance (Stein and Baerwald 2014), dosage (Brown, Milavetz et al. 2013), route of administration (Brown, Milavetz et al. 2013), etc.

Several of these factors which may alter drug effectiveness were controlled for by design; the strength case-crossover studies are that all fixed confounders are controlled. Despite this strength, this study has several distinct limitations. First, certain time-varying covariates were not adjusted for as many 
of them were immeasurable. For example, driving exposure (i.e. the amount that an individual drives), is a known and important confounder of traffic studies (Blanchard, Myers et al. 2010) and could not be accounted for in this analysis because it is absent from the medical records. Second, there were limitations associated with the medical records. Much of the information collected was self-reported by the patient or their legal guardian and subject to recall bias. Some of the information, such as medication duration, may have been incomplete or was simply not documented. Also, the accuracy of E-codes for identifying potential cases in this analysis may have been lacking (Bowman, Aitken 2011); therefore some potential cases may have been unnecessarily excluded particularly if the patient was not coded properly. Third, patient behavior in terms of their medication usage was unknown; it was impossible to determine if the patient was taking their medication as prescribed by their healthcare provider. Fourth, the sample sizes in this analysis were often small and many statistical tests were likely under-powered. Also, several regression analyses were run, so statistical significance could have been achieved by chance alone. Fifth, as this study was conducted in only one state, the findings may not be generalizable to other locations. Sixth, as previously mentioned, disease-medication relationships are often difficult to distinguish. The findings of this analysis are not suggesting that the medications were the cause of the motor vehicle collision; the findings are associative. Lastly, a known weakness of case-crossover studies is that they are subject to selection bias in terms of choosing proper control periods (Greenland 1996). Future research may involve replicating this study in a larger population that is nationally representative or in a different age group.

\section{Conclusion}

This analysis sought to explore which licit drugs were most common, if illicit drug use was prevalent, and which licit drugs were associated with an increased risk of motor vehicle collision among West Virginia drivers 65 years of age or older using a case-crossover approach. It was determined that analgesics, cardiovascular and gastrointestinal drugs were prevalent in this population. While few drivers were tested, illicit drug use was uncommon. Those taking frequently prescribed medications, such as 
Tramadol, were at a significantly increased risk of motor vehicle collision while taking these substances during the 14 day risk period compared to control periods. Many medications were trending towards an increased risk of motor vehicle collision but statistical models were likely under-powered. Future research in this area is necessary as different medications may pose more risk to patient safety than others. The association between medication use and the risk of motor vehicle collisions is particularly important as more drivers are living longer and often choose to maintain their mobility later in life despite their medical conditions. 


\section{Chapter 5}

\section{Discussion}

\subsection{Summary of key findings}

This dissertation research sought to explore licit and illicit drug usage among drivers 65 years of age and older in the United States via a systematic review of the literature (Chapter 2), a cross-sectional analysis of traffic fatality database (Chapter 3), and a case-crossover study of senior drivers admitted to medical treatment following a motor vehicle collision (Chapter 4). Each study contributed a different key piece of information in which the other two studies alone could not answer.

The findings presented in Chapter 2 helped frame the research pursued in Chapters 3 and 4 by determining which individual medications were associated with the increased odds/risk of motor vehicle collision and/or impaired driving ability. The key finding of Chapter 2 was that among the 53 specific medications investigated by the 27 studies included in the review, 15 medications (28.3\%) were associated with an increased risk of motor vehicle collision. The medications that were associated with an increased risk of collision were: Buprenorphine, Codeine, Dihydrocodeine, Methadone, Tramadol, Levocitirizine, Diazepam, Flunitrazepam, Flurazepam, Lorazepam, Temazepam, Triazolam, Carisoprodol, Zolpidem, and Zopiclone. The findings of this review illustrated that certain medications, even within the same class or drug category, may be more associated with crash risk than others.

The cross-sectional analysis presented in Chapter 3 provided a unique perspective of drug prevalence among fatally injured drivers 65 years of age and older across the United States. Chapter 3 helped determine which individual, broad groups, and combinations of licit and illicit drugs senior drivers had actually consumed prior to their motor vehicle collision; the prevalence of these drugs was compared to a referent population of middle-aged drivers. The key finding of Chapter 3 was that overall licit and illicit drug usage was not common among fatally injured senior drivers. Those who did test positive $(17.8 \%)$ tended to test positive for 'other drugs', narcotic analgesics and/or benzodiazepines. Based on the limitation of the FARS data, it could not be determined what the 'other drugs' were; it was 
hypothesized that these were cardiovascular or gastrointestinal medications as these are the most common medication types that older adults consume based on retail drug sales (Fulton and Allen 2005).

The case-crossover study presented in Chapter 4 enabled the characterization of drug use and the risk of motor vehicle collision posed by individual medications among drivers in West Virginia 65 years of age and older (as indicated by their electronic medical records). The key findings to Chapter 4 were that analgesic, cardiovascular and gastrointestinal drugs were common. Few drivers were screened for drugs at time of admittance to medical treatment. Of those testing positive, benzodiazepines and opiates were the most common. As for the association between medications and motor vehicle collision risk, senior drivers consuming Tramadol were at a significantly increased risk of motor vehicle collision if they consumed this medication 14 days prior to their motor vehicle collision compared to other control periods. Numerous other medications, such as Clopidogrel, Gabapentin, Citalopram, Insulin, Hydrochlorothiazide, Metoprolol, Zolpidem, and Nitroglycerine, were trending towards an increased risk of motor vehicle collision though were not statistically significant.

\subsection{Significance of study findings}

Collectively, the findings of this dissertation research pose numerous clinical and research implications. From a clinical perspective, the findings raise concerns regarding patients' safety, awareness and/or education. Based on the findings of Chapters 2 and 4, it was determined that Buprenorphine, Codeine, Dihydrocodeine, Methadone, Tramadol, Levocitirizine, Diazepam, Flunitrazepam, Flurazepam, Lorazepam, Temazepam, Triazolam, Carisoprodol, Zolpidem, and Zopiclone may pose an increased risk of motor vehicle collision to drivers. Many of these medications are widely prescribed to seniors. Also, among drivers who did test positive for drugs either at death or upon admittance for medical treatment, illicit substances were infrequent whereas prescription medications, such as benzodiazepines and narcotics, were common. Therefore, patient awareness and educational efforts are needed to ensure patients who continue to drive late in life are aware of the risk that their medications may pose to their driving ability. Education of older patients is particularly important because it is known that health literacy declines with age, which is possibly attributed to cognitive 
declines (Baker, Gazmararian et al. 2000). Also, it is known that patient education of this age group is lacking. A study conducted by Kelly et al showed that many patients were not given proper instruction from their primary health care provider to limit or stop driving due to a recent diagnosis and/or

prescription for a medication which could be driver-impairing (Kelly, Warke et al. 1999). Interventional efforts in this area appear abundant.

The findings of this dissertation also have research implications. It is possible that individual medications within the same 'type' may pose different risks to patients then others. This was seen in Chapter 4 with anticoagulants and antihypertensive medications where certain drugs within these categories were trending towards being protective against motor vehicle collision while others were not. While the exact reason as to why this was observed is unknown. These findings may suggest that the pharmacokinetic properties of these drugs augment motor vehicle collision risk. Further exploration of this issue is necessary.

Individually, the findings of this dissertation research are also significant because they contribute to the extant gaps in the literature. As no reviews have looked at the risk of individual medications on motor vehicle collision, the systematic review answered this question. The cross-sectional analysis characterized drug use among older adult drivers in the U.S. which has not been done to date. The casecrossover study contributed to the limited information that exists regarding Tramadol; comparing this study to others, it appears that the effects of Tramadol are not age-dependent. Also, the case-crossover study investigated individual medication use and the risk of motor vehicle collision. To the authors' knowledge, this has not been done to date.

\subsection{Strengths and limitations}

The strength of the present study is that it utilized different approaches (i.e. a systematic review, a cross-sectional study using national data, and a case-crossover study) to explore overall drug use and the relationship between licit drug use and motor vehicle collision among senior drivers. Each study was a complement of the others. Despite this strength, as discussed previously in Chapters 2 thru 4, each study had their own innate limitations. Briefly, the systematic review was subject to publication bias. The 
cross-sectional analysis was subject to limitations surrounding the FARS data. The case-crossover study was subject to small sample sizes and the completeness of the electronic medical records. Despite these limitations unique to each study, the overarching limitation of this dissertation research involves confounding. First, the relationships between disease, the natural aging process, and medication use are extremely convoluted. It is nearly impossible to differentiate which is the most 'responsible' for declines in driving ability and/or increased motor vehicle collision risk. It is entirely possible that the natural aging process or the underlying medical condition in which the medications were prescribed caused the individuals in this analysis to be involved in their motor vehicle collisions. It is possible that the collisions could have been attributed to a combination of the three. Second, driving exposure (i.e. the amount someone drives in the time period of interest), a known confounder of traffic studies (Blanchard, Myers et al. 2010), and 'self-regulation' could not be assessed. It is a known phenomenon among older adults that they will regulate where and how much they drive when they perceive their driving ability to be compromised (D'Ambrosio, Donorfio et al. 2008). Driving exposure and self-regulation could not be accounted for in any of the studies. Also, driver fault could not be adequately assessed in Chapter 3 or Chapter 4, which could also have been a confounder. Despite the efforts taken to control for confounding, it is virtually impossible to eliminate entirely which may have biased the results.

\subsection{Future research}

Future research in this area of study is plentiful. Future studies may involve trying to separate the effects of confounding surrounding the relationship between the aging process, chronic medical conditions, and medications. Future studies could also involve repeating the case-crossover study in a larger, more nationally representative population. Other studies could involve seeing if age mediates the relationships seen in this analysis by repeating the study in a larger population in different age groups. 


\section{References}

\section{Chapter 1}

Al-Hassani, S. B. and N. M. Alotaibi (2014). "The Impact of Driving Cessation on Older Kuwaiti Adults: Implications to Occupational Therapy." Occupational Therapy in Health Care 28(3): 264-276.

Amato, J.-N., S. Marie, et al. (2013). "Effects of three therapeutic doses of codeine/paracetamol on driving performance, a psychomotor vigilance test, and subjective feelings." Psychopharmacology 228(2): 309-320.

Anstey, K. J. and J. Wood (2011). "Chronological age and age-related cognitive deficits are associated with an increase in multiple types of driving errors in late life." Neuropsychology 25(5): 613-621.

Antin, J. F., T. E. Lockhart, et al. (2012). "Comparing the impairment profiles of older drivers and nondrivers: Toward the development of a fitness-to-drive model." Safety Science 50(2): 333-341.

Asbridge, M., R. Mann, et al. (2014). "Cannabis and traffic collision risk: Findings from a case-crossover study of injured drivers presenting to emergency departments." International Journal of Public Health 59(2): 395-404.

Augustijns, P., B. Wuyts, et al. (2014). "A review of drug solubility in human intestinal fluids: implications for the prediction of oral absorption." Eur J Pharm Sci 57: 322-332.

Bachs, L. C., A. Engeland, et al. (2009). "The risk of motor vehicle accidents involving drivers with prescriptions for codeine or tramadol." Clinical Pharmacology and Therapeutics 85(6): 596-599.

Ball, K., J. D. Edwards, et al. (2010). "Cognitive Training Decreases Motor Vehicle Collision Involvement of Older Drivers BALL ET AL. COGNITIVE TRAINING DECREASES CRASH RATE." Journal of the American Geriatrics Society 58(11): 2107-2113.

Barbone, F., A. D. McMahon, et al. (1998). "Association of road-traffic accidents with benzodiazepine use." Lancet (England) 352(Oct 24): 1331-1336.

Beaussart, M., J. Beaussart-Defaye, et al. (1997). "Epileptic drivers - A study of 1,089 patients." Medicine and Law 16(2): 295-306.

Becton, J. B., H. J. Walker, et al. (2014). "Generational differences in workplace behavior." Journal of Applied Social Psychology 44(3): 175-189.

Bedard, M. (2000). Older drivers and fatal motor vehicle crashes, UMI Dissertation Services, ProQuest Information and Learning, Ann Arbor, MI.

Bedard, M., S. Dubois, et al. (2007). "The impact of cannabis on driving." Can J Public Health 98(1): 611.

Berta, E., M. Harangi, et al. (2014). "Effect of thyroid hormone status and concomitant medication on statin induced adverse effects in hyperlipidemic patients." Pharmazie 69(6): 420-423. 
Beynon, C. M. (2009). "Drug use and ageing: older people do take drugs!" Age and Ageing 38(1): 8-10.

Bonnel, W. B. (1999). "Giving up the car: older women's losses and experiences." J Psychosoc Nurs Ment Health Serv 37(5): 10-15.

Bosker, W. M., K. P. Kuypers, et al. (2012). "Medicinal Delta(9) -tetrahydrocannabinol (dronabinol) impairs on-the-road driving performance of occasional and heavy cannabis users but is not detected in Standard Field Sobriety Tests." Addiction 107(10): 1837-1844.

Bosker, W. M., K. P. C. Kuypers, et al. (2012). "MDMA (ecstasy) effects on actual driving performance before and after sleep deprivation, as function of dose and concentration in blood and oral fluid." Psychopharmacology 222(3): 367-376.

Bramness, J. G., S. Skurtveit, et al. (2012). "An increased risk of motor vehicle accidents after prescription of methadone." Addiction 107(5): 967-972.

Bramness, J. G., S. Skuytveit, et al. (2008). "Minor increase in risk of road traffic accidents after prescriptions of antidepressants: A study of population registry data in Norway." Journal of Clinical Psychiatry 69(Jul): 1099-1103.

Braver, E. R. and R. E. Trempel (2004). "Are older drivers actually at higher risk of involvement in collisions resulting in deaths or non-fatal injuries among their passengers and other road users?" Inj Prev 10(1): 27-32.

Brookhuis, K. A., E. R. Volkerts, et al. (1990). "Repeated dose effects of lormetazepam and flurazepam upon driving performance." European Journal Of Clinical Pharmacology 39(1): 83-87.

Brown, T., G. Milavetz, et al. (2013). Alcohol, drugs and driving: Implications for evaluating driver impairment. 57th Annual Scientific Conference of the Association for the Advancement of Automotive Medicine, Quebec City, QC.

Brzaković, B. B., S. D. Vezmar Kovačević, et al. (2012). "Impact of age, weight and concomitant treatment on lamotrigine pharmacokinetics." Journal of Clinical Pharmacy \& Therapeutics 37(6): 693-697.

Bushardt, R. L., E. B. Massey, et al. (2008). "Polypharmacy: misleading, but manageable." $\underline{\text { Clin Interv }}$ Aging 3(2): 383-389.

Byas-Smith, M. G., S. L. Chapman, et al. (2005). "The effect of opioids on driving and psychomotor performance in patients with chronic pain." The Clinical Journal Of Pain 21(4): 345-352.

Centers for Disease Control and Prevention (2003). "Trends in aging--United States and worldwide." MMWR Morb Mortal Wkly Rep 52(6): 101-104, 106.

Chang, C. M., E. C. H. Wu, et al. (2013). "Psychotropic drugs and risk of motor vehicle accidents: A population-based case-control study." British Journal of Clinical Pharmacology 75(4): 11251133. 
Cheung, I. and A. T. McCartt (2011). "Declines in fatal crashes of older drivers: changes in crash risk and survivability." Accid Anal Prev 43(3): 666-674.

Chu, T. (2014). "Gender differences in pharmakokinectics." U.S. Pharmacist 39(9): 40-43.

Cingi, C., S. Z. Toros, et al. (2013). "Effect of grapefruit juice on bioavailability of montelukast." The Laryngoscope 123(4): 816-819.

Classen, S., B. Brumback, et al. (2014). "Driving errors in Parkinson's disease: moving closer to predicting on-road outcomes." Am J Occup Ther 68(1): 77-85.

Conen, S., E. L. Theunissen, et al. (2011). "Acute and subchronic effects of bilastine (20 and $40 \mathrm{mg}$ ) and hydroxyzine $(50 \mathrm{mg})$ on actual driving performance in healthy volunteers." Journal of Psychopharmacology 25(11): 1517-1523.

Corsenac, P., E. Lagarde, et al. (2012). "Road traffic crashes and prescribed methadone and buprenorphine: A french registry-based case-control study." Drug \& Alcohol Dependence 123(13): 91-97.

Coupland, C. A. C., P. Dhiman, et al. (2011). "A study of the safety and harms of antidepressant drugs for older people: A cohort study using a large primary care database." Health Technology Assessment 15(28): 5-218.

Cox, D. J., W. C. Quillian, et al. (1998). "Evaluating driving performance of outpatients with Alzheimer disease." Journal of the American Board of Family Practice 11(4): 264-271.

Dall, T. M., P. D. Gallo, et al. (2013). "An Aging Population And Growing Disease Burden Will Require ALarge And Specialized Health Care Workforce By 2025." Health Affairs 32(11): 2013-2020.

Daly, A. K. (2014). "Is there a need to teach pharmacogenetics?" Clin Pharmacol Ther 95(3): 245-247.

Daurat, A., P. Sagaspe, et al. (2013). "Lorazepam impairs highway driving performance more than heavy alcohol consumption." Accident Analysis \& Prevention 60: 31-34.

Dawson, J. D., E. Y. Uc, et al. (2010). "Neuropsychological predictors of driving errors in older adults." Journal of the American Geriatrics Society 58(6): 1090-1096.

De Gier, J. J., B. J. Hart, et al. (1981). "Psychomotor performance and real driving performance of outpatients receiving diazepam." Psychopharmacology 73(4): 340-344.

Dellinger, A. M., M.-j. Kresnow, et al. (2004). "Risk to self versus risk to others: How do older drivers compare to others on the road?" American Journal of Preventive Medicine 26(3): 217-221.

Dickerson, A. E., T. Reistetter, et al. (2013). "The perception of meaningfulness and performance of instrumental activities of daily living from the perspectives of the medically at-risk older adults and their caregivers." J Appl Gerontol 32(6): 749-764.

Duchek, J. M., D. B. Carr, et al. (2003). "Longitudinal Driving Performance in Early-Stage Dementia of the Alzheimer Type." Journal of the American Geriatrics Society 51(10): 1342. 
Eby, D. W., N. M. Silverstein, et al. (2012). "Driving behaviors in early stage dementia: A study using invehicle technology." Accident Analysis \& Prevention 49(0): 330-337.

Edwards, G. and P. J. Quartaro (1978). "Heroin addiction and road traffic accidents." British Medical Journal (England) 2(Dec 16): 1710.

Edwards, J. D., P. B. Delahunt, et al. (2009). "Cognitive Speed of Processing Training Delays Driving Cessation." The Journals of Gerontology Series A: Biological Sciences and Medical Sciences 64A(12): 1262-1267.

Edwards, J. D., M. Perkins, et al. (2009). "Driving Status and Three-Year Mortality Among CommunityDwelling Older Adults." The Journals of Gerontology Series A: Biological Sciences and Medical Sciences 64A(2): 300-305.

England, A., S. Skurtveit, et al. (2007). "Risk of Road Traffic Accidents Associated With the Prescription of Drugs: A Registry-Based Cohort Study." Annals of epidemiology 17(8): 597 - 602.

Ferguson, D. M. and L. J. Horwood (2001). "CANNABIS USE AND TRAFFIC ACCIDENTS IN A BIRTH COHORT OF YOUNG ADULTS." Accident Analysis \& Prevention 33(6): p. 703-711.

Fitten, L., K. M. Perryman, et al. (1995). "Alzheimer and vascular dementias and driving: A prospective road and laboratory study." JAMA 273(17): 1360-1365.

Foley, D. J., R. B. Wallace, et al. (1995). "Risk factors for motor vehicle crashes among older drivers in a rural community." J Am Geriatr Soc 43(7): 776-781.

Fonda, S. J., R. B. Wallace, et al. (2001). "Changes in Driving Patterns and Worsening Depressive Symptoms Among Older Adults." The Journals of Gerontology Series B: Psychological Sciences and Social Sciences 56(6): S343-S351.

Frittelli, C., D. Borghetti, et al. (2009). "Effects of Alzheimer's disease and mild cognitive impairment on driving ability: A controlled clinical study by simulated driving test." International Journal of Geriatric Psychiatry 24(3): 232-238.

Fulton, M. M. and E. R. Allen (2005). "Polypharmacy in the elderly: a literature review." J Am Acad Nurse Pract 17(4): 123-132.

Gagliardi, C., F. Marcellini, et al. (2010). "Associations of personal and mobility resources with subjective well-being among older adults in Italy and Germany." Archives of Gerontology and Geriatrics 50(1): 42-47.

George, C. F. P., A. C. Boudreau, et al. (1996). "Simulated driving performance in patients with obstructive sleep apnea." American Journal of Respiratory and Critical Care Medicine 154(1): 175-181. 
Gieteling, E. W., M. S. Bakker, et al. (2012). "Impaired driving simulation in patients with Periodic Limb Movement Disorder and patients with Obstructive Sleep Apnea Syndrome." Sleep Medicine 13(5): $517-523$.

Gomes, T., D. A. Redelmeier, et al. (2013). "Opioid dose and risk of road trauma in Canada: A population-based study." JAMA Internal Medicine 173(3): 196-201.

Gramaekers, J. G., H. W. J. Robbe, et al. (2000). "Marijuana, alcohol and actual driving performance." Human Psychopharmacology: Clinical \& Experimental 15(7): 551-558.

Green, K. A., G. McGwin, et al. (2013). "Associations Between Visual, Hearing, and Dual Sensory Impairments and History of Motor Vehicle Collision Involvement of Older Drivers." Journal of the American Geriatrics Society 61(2): 252-257.

Gresset, J. and F. Meyer (1994). "Risk of Automobile Accidents Among Elderly Drivers with Impairments or Chronic Diseases." Canadian Journal of Public Health / Revue Canadienne de Sante'e Publique 85(4): 282-285.

Guibert, R., L. Potvin, et al. (1998). "Are drivers with CVD more at risk for motor vehicle crashes? Study of men aged 45 to 70." Can Fam Physician 44: 770-776.

Gustavsen, I., J. G. Bramness, et al. (2008). "Road traffic accident risk related to prescriptions of the hypnotics zopiclone, zolpidem, flunitrazepam and nitrazepam." Sleep Medicine 9(8): 818-822.

Hansotia, P. and S. K. Broste (1991). "The effect of epilepsy or diabetes mellitus on the risk of automobile accidents." N Engl J Med 324(1): 22-26.

Haustein, S. and A. Siren (2014). "Seniors' unmet mobility needs - how important is a driving licence?" Journal of Transport Geography 41(0): 45-52.

Haymes, S. A., R. P. Leblanc, et al. (2007). "Risk of falls and motor vehicle collisions in glaucoma." Invest Ophthalmol Vis Sci 48(3): 1149-1155.

Hemmelgarn, B., L. E. Lévesque, et al. (2006). "Anti-diabetic drug use and the risk of motor vehicle crash in the elderly." Canadian Journal of Clinical Pharmacology 13(1): e112-e120.

Hickson, L., J. Wood, et al. (2010). "Hearing impairment affects older people's ability to drive in the presence of distracters." Journal of the American Geriatrics Society 58(6): 1097-1103.

Higgins, J. P., S. W. Wright, et al. (1996). "Alcohol, the elderly, and motor vehicle crashes." Am J Emerg Med 14(3): 265-267.

Hindmarch, I. (1976). "The effects of the sub-chronic administration of an anti-histamine, clemastine, on tests of car driving ability and psychomotor performance." Curr Med Res Opin 4(3): 197-206.

Hindmarch, I. and A. C. Gudgeon (1980). "The effects of clobazam and lorazepam on aspects of psychomotor performance and car handling ability." British Journal Of Clinical Pharmacology 10(2): $145-150$. 
Hindmarch, I., G. W. Hanks, et al. (1977) "Clobazam, a 1,5-benzodiazepine, and car-driving ability." British journal of clinical pharmacology, 573-578.

Hindmarch, I. and Z. Subhan (1983). "The effects of midazolam in conjunction with alcohol on sleep, psychomotor performance and car driving ability." International Journal Of Clinical Pharmacology Research 3(5): 323-329.

Hindmarch, I., L. Trick, et al. (2005) "A double-blind, placebo- and positive-internal-controlled (alprazolam) investigation of the cognitive and psychomotor profile of pregabalin in healthy volunteers." Psychopharmacology, 133-143 DOI: 10.1007/s00213-005-0172-7.

Horswill, M. S., N. A. Pachana, et al. (2009). "A comparison of the hazard perception ability of matched groups of healthy drivers aged 35 to 55,65 to 74 , and 75 to 84 years." J Int Neuropsychol Soc 15(5): 799-802.

Hours, M., E. Fort, et al. (2008). "Diseases, consumption of medicines and responsibility for a road crash: A case-control study." Accident Analysis \& Prevention 40(5): 1789-1796.

Hours, M., E. Fort, et al. (2008). "Diseases, consumption of medicines and responsibility for a road crash: a case-control study." Accid Anal Prev 40(5): 1789-1796.

Innominato, P. F., V. P. Roche, et al. (2014). "The circadian timing system in clinical oncology." Ann Med 46(4): 191-207.

Ivers, R. Q., P. Mitchell, et al. (1999). "Sensory Impairment and Driving: The Blue Mountains Eye Study." American Journal of Public Health 89(1): 85-85.

Johnson, J. E. (1999). "Urban older adults and the forfeiture of a driver's license." J Gerontol Nurs 25(12): 12-18.

Jorgensen, T., S. Johansson, et al. (2001). "Prescription drug use, diagnoses, and healthcare utilization among the elderly." Ann Pharmacother 35(9): 1004-1009.

Joy, M. S., R. F. Frye, et al. (2014). "In vivo alterations in drug metabolism and transport pathways in patients with chronic kidney diseases." Pharmacotherapy 34(2): 114-122.

Jusko, W. J. (2013). "Moving from basic toward systems pharmacodynamic models." J Pharm Sci 102(9): 2930-2940.

Karakontaki, F., S.-A. Gennimata, et al. (2013). "Driving-Related Neuropsychological Performance in Stable COPD Patients." Pulmonary Medicine: 1-10.

Keall, M. D. and W. J. Frith (2004). "Older driver crash rates in relation to type and quantity of travel." Traffic Inj Prev 5(1): 26-36.

Keall, M. D. and W. J. Frith (2006). "Characteristics and Risks of Drivers with Low Annual Distance Driven." Traffic Injury Prevention 7(3): 248-255. 
King, M. D., T. M. Meuser, et al. (2011). "Decoding the Miss Daisy Syndrome: an examination of subjective responses to mobility change." J Gerontol Soc Work 54(1): 29-52.

Koepsell, T. D., M. E. Wolf, et al. (1994). "Medical conditions and motor vehicle collision injuries in older adults." J Am Geriatr Soc 42(7): 695-700.

Kuypers, K. P. C., S.-A. Legrand, et al. (2012). "A Case-Control Study Estimating Accident Risk for Alcohol, Medicines and Illegal Drugs." PLoS ONE 7(8): 1-9.

Kuypers, K. P. C., N. Samyn, et al. (2006). "MDMA and alcohol effects, combined and alone, on objective and subjective measures of actual driving performance and psychomotor function." Psychopharmacology 187(4): 467-475.

Laing, S. J. (2003). Study of age differences in accident rates at intersections in British Columbia, UMI Dissertation Services, ProQuest Information and Learning, Ann Arbor, MI.

Langford, J., M. Bohensky, et al. (2008). "Do Older Drivers Pose a Risk to Other Road Users?" Traffic Injury Prevention 9(3): 181-189.

Langford, J., J. L. Charlton, et al. (2013). "Findings from the Candrive/Ozcandrive study: Low mileage older drivers, crash risk and reduced fitness to drive." Accident Analysis \& Prevention 61(0): 304-310.

Langford, J., S. Koppel, et al. (2008). "In defence of the 'low-mileage bias'." Accident Analysis \& Prevention 40(6): 1996-1999.

Lasebikan, V. O. (2010). "Is cannabis use related to road crashes? A study of long distance commercial drivers in Nigeria." African Journal of Drug and Alcohol Studies 9(1): 23-32.

Laumon, B., B. Gadegbeku, et al. (2005). "Cannabis intoxication and fatal road crashes in France: population based case-control study." BMJ 331(7529): 1371.

Laurell, H. and J. Tornros (1986). "The carry-over effects of triazolam compared with nitrazepam and placebo in acute emergency driving situations and in monotonous simulated driving." Acta Pharmacol Toxicol (Copenh) 58(3): 182-186.

Lefrancois, R. and M. D'Amours (1997). "Exposure and risk factors among elderly drivers: a case-control study." Accident Analysis and Prevention 29(3): 267-275.

LeRoy, A. A. and M. L. Morse (2008). Multiple Medications and Vehicle Crashes: Analysis of Databases. National Highway Traffic Safety Administration. Washington.

Leufkens, T. R., A. Vermeeren, et al. (2007) "Cognitive, psychomotor and actual driving performance in healthy volunteers after immediate and extended release formulations of alprazolam $1 \mathrm{mg} . "$ Psychopharmacology, 951-959 DOI: 10.1007/s00213-006-0669-8. 
Leufkens, T. R. M., J. S. Lund, et al. (2009). "Highway driving performance and cognitive functioning the morning after bedtime and middle-of-the-night use of gaboxadol, zopiclone and zolpidem." Journal Of Sleep Research 18(4): 387-396.

Leufkens, T. R. M. and A. Vermeeren (2009). "Highway driving in the elderly the morning after bedtime use of hypnotics: A comparison between temazepam $20 \mathrm{mg}$, zopiclone $7.5 \mathrm{mg}$, and placebo." Journal of Clinical Psychopharmacology 29(5): 432-438.

Leveille, S. G., D. M. Buchner, et al. (1994). "Psychoactive medications and injurious motor vehicle collisions involving older drivers." Epidemiology 5(6): 591-598.

Li, G., J. E. Brady, et al. (2013). "Drug use and fatal motor vehicle crashes: A case-control study." Accident Analysis \& Prevention 60: 205-210.

Li, G., E. R. Braver, et al. (2003). "Fragility versus excessive crash involvement as determinants of high death rates per vehicle-mile of travel among older drivers." Accident Analysis and Prevention 35(2): 227-235.

Lings, S. and E. Dupont (1992). "Driving with Parkinson's disease. A controlled laboratory investigation." Acta Neurologica Scandinavica 86(1): 33-39.

Lonnen, K. F., R. J. Powell, et al. (2008). "Road traffic accidents and diabetes: insulin use does not determine risk." Diabetic Medicine 25(5): 578-584.

Lotfipour, S. and F. Vaca (2007). "Commentary: Polypharmacy and older drivers: beyond the doors of the emergency department (ED) for patient safety." Ann Emerg Med 49(4): 535-537.

Lundqvist, A., B. Gerdle, et al. (2000). "Neuropsychological aspects of driving after a stroke-in the simulator and on the road." Applied Cognitive Psychology 14(2): 135-150.

Majdzadeh, R., A. Feiz-Zadeh, et al. (2009). "Opium consumption and the risk of traffic injuries in regular users: A case-crossover study in an emergency department." Traffic Injury Prevention 10(4): 325-329.

MaloneBeach, E. E. and K. L. Langeland (2010). "Boomers' Prospective Needs for Senior Centers and Related Services: A Survey of Persons 50-59." J Gerontol Soc Work 54(1): 116-130.

Mann, R. E., E. Adlaf, et al. (2007). "Cannabis use and self-reported collisions in a representative sample of adult drivers." Journal of Safety Research 38(6): 669-674.

Mann, R. E., G. Stoduto, et al. (2010). Self-reported collision risk associated with cannabis use and driving after cannabis use among Ontario adults. Traffic Inj Prev. England. 11: 115-122.

Markert, C., P. Ngui, et al. (2014). "Influence of St. John's wort on the steady-state pharmacokinetics and metabolism of bosentan." Int J Clin Pharmacol Ther 52(4): 328-336. 
Marottoli, R. A., C. F. M. de Leon, et al. (2000). "Consequences of Driving Cessation: Decreased Out-ofHome Activity Levels." The Journals of Gerontology Series B: Psychological Sciences and Social Sciences 55(6): S334-S340.

Martin, L. G., V. A. Freedman, et al. (2009). "Health and Functioning Among Baby Boomers Approaching 60." The Journals of Gerontology Series B: Psychological Sciences and Social Sciences 64B(3): 369-377.

Massie, D. L., K. L. Campbell, et al. (1995). "Traffic accident involvement rates by driver age and gender." Accid Anal Prev 27(1): 73-87.

McGwin, G., Jr., R. V. Sims, et al. (1999). "Diabetes and automobile crashes in the elderly. A populationbased case-control study." Diabetes Care 22(2): 220-227.

McGwin, G., Jr., R. V. Sims, et al. (2000). "Relations among chronic medical conditions, medications, and automobile crashes in the elderly: a population-based case-control study." Am J Epidemiol 152(5): 424-431.

McGwin, J. G. and D. B. Brown (1999). "Characteristics of traffic crashes among young, middle-aged, and older drivers." Accident Analysis \& Prevention 31(3): 181-198.

McGwin Jr, G., V. Chapman, et al. (2000). "Visual risk factors for driving difficulty among older drivers." Accident Analysis \& Prevention 32(6): 735-744.

McKnight, A. J. and A. S. McKnight (1999). "Multivariate analysis of age-related driver ability and performance deficits." Accident Analysis \& Prevention 31(5): 445-454.

Mercier-Guyon, C., J. Lejay, et al. (1999). "Comparative Study of the Effects of Captodiamine and Lorazepam on Car Driving Ability." Clinical Drug Investigation 17(6): 451-459.

Mermin, G. B. T., R. W. Johnson, et al. (2007). "Why Do Boomers Plan to Work Longer?" The Journals of Gerontology Series B: Psychological Sciences and Social Sciences 62(5): S286-S294.

Mets, M. A., J. M. Vries, et al. (2011) "Next-day effects of ramelteon (8 mg), zopiclone (7.5 mg), and placebo on highway driving performance, memory functioning, psychomotor performance, and mood in healthy adult subjects." Sleep, 1327-1334 DOI: 10.5665/SLEEP.1272.

Meuleners, L. B., J. Duke, et al. (2011). "Psychoactive Medications and Crash Involvement Requiring Hospitalization for Older Drivers: A Population-Based Study." Journal of the American Geriatrics Society 59(9): 1575-1580.

Mezuk, B. and G. W. Rebok (2008). "Social Integration and Social Support Among Older Adults Following Driving Cessation." The Journals of Gerontology Series B: Psychological Sciences and Social Sciences 63(5): S298-S303. 
Moták, L., L. Bayssac, et al. (2014). "Naturalistic conversation improves daytime motorway driving performance under a benzodiazepine: A randomised, crossover, double-blind, placebo-controlled study." Accident Analysis and Prevention 67: 61-66.

Mu-Chen, L., B. Joanne E., et al. (2012). "Marijuana Use and Motor Vehicle Crashes." Epidemiologic reviews 34(1): 65-72.

Mulgrew, A. T., G. Nasvadi, et al. (2008). "Risk and severity of motor vehicle crashes in patients with obstructive sleep apnoea/hypopnoea." Thorax 63(6): 536-541.

Mura, P., B. Brunet, et al. (2006). "Cannabis and road crashes: a survey of recent French studies." Annales Pharmaceutiques Francaises (France) 64(Mar): 192-196.

Myrick, B. E. (2001). Accident experience of rural elderly drivers in New Brunswick, UMI Dissertation Services, ProQuest Information and Learning, Ann Arbor, MI.

National Highway Traffic Safety Administration (2013). Traffic safety facts, older population. Washington.

Neutel, I. (1998). "Benzodiazepine-related traffic accidents in young and elderly drivers." Human Psychopharmacology: Clinical \& Experimental 13: S115-S123.

O'Hanlon, J. F., T. W. Haak, et al. (1982). "Diazepam impairs lateral position control in highway driving." Science 217(4554): 79-81.

O'Hanlon, J. F., H. W. J. Robbe, et al. (1998). "Venlafaxine's effects on healthy volunteers' driving, psychomotor, and vigilance performance during 15-day fixed and incremental dosing regimens." Journal of Clinical Psychopharmacology 18(3): 212-221.

Orriols, L., P. Philip, et al. (2011). "Benzodiazepine-like hypnotics and the associated risk of road traffic accidents." Clinical Pharmacology and Therapeutics 89(4): 595-601.

Orriols, L., R. Queinec, et al. (2012). "Risk of injurious road traffic crash after prescription of antidepressants." Journal of Clinical Psychiatry 73(8): 1088-1094.

Orriols, L., M. Wilchesky, et al. (2013). "Prescription of antidepressants and the risk of road traffic crash in the elderly: a case-crossover study." British Journal of Clinical Pharmacology 76(5): 810-815.

Owsley, C., G. McGwin Jr, et al. (2002). "Impact of cataract surgery on motor vehicle crash involvement by older adults." Journal of the American Medical Association 288(7): 841-849.

Owsley, C., B. Stalvey, et al. (1999). "Older Drivers and Cataract: Driving Habits and Crash Risk." The Journals of Gerontology Series A: Biological Sciences and Medical Sciences 54(4): M203-M211.

Oxley, J., J. Langford, et al. (2010). "The safe mobility of older drivers: a challenge for urban road designers." Journal of Transport Geography 18: 642-648.

Pallin, D. J., M. B. Allen, et al. (2013). "Population Aging And Emergency Departments: Visits Will Not Increase, Lengths-Of-Stay And Hospitalizations Will." Health Affairs 32(7): 1306-1312. 
Pallin, D. J., J. A. Espinola, et al. (2014). "US population aging and demand for inpatient services." J Hosp Med 9(3): 193-196.

Pekmezaris, R., A. Kozikowski, et al. (2013). "Aging in Suburbia: An Assessment of Senior Needs." Educational Gerontology 39(5): 355-365.

Potter, P. C., J. M. Schepers, et al. (2003) "The effects of fexofenadine on reaction time, decision-making, and driver behavior." Annals of allergy, asthma \& immunology, 177-181 DOI: 10.1016/S10811206(10)62174-6.

Pulido, J., G. Barrio, et al. (2011). "Association between cannabis and cocaine use, traffic injuries and use of protective devices." European Journal of Public Health 21(6): 753-755.

Ragland, D. R., W. A. Satariano, et al. (2005). "Driving Cessation and Increased Depressive Symptoms." The Journals of Gerontology Series A: Biological Sciences and Medical Sciences 60(3): 399-403.

Ramaeker, J. G. and J. F. O'Hanlon (1994). "Acrivastine, terfenadine and diphenhydramine effects on driving performance as a function of dose and time after dosing." European Journal of Clinical Pharmacology 47(3): 261-266.

Ramaekers, J. G., M. Ansseau, et al. (1997) "Considering the P450 cytochrome system as determining combined effects of antidepressants and benzodiazepines on actual driving performance of depressed outpatients." International clinical psychopharmacology, 159-169.

Ramaekers, J. G., K. P. Kuypers, et al. (2006) "Stimulant effects of 3,4-methylenedioxymethamphetamine (MDMA) $75 \mathrm{mg}$ and methylphenidate $20 \mathrm{mg}$ on actual driving during intoxication and withdrawal." Addiction (Abingdon, England), 1614-1621 DOI: 10.1111/j.13600443.2006.01566.x.

Ramaekers, J. G., N. D. Muntjewereff, et al. (1998). "Effects of nocturnal doses of mirtazapine and mianserin on sleep and on daytime psychomotor and driving performance in young, healthy volunteers." Human Psychopharmacology: Clinical \& Experimental 13: S87-S97.

Ramaekers, J. G., N. D. Muntjewerff, et al. (1995). "A comparative study of acute and subchronic effects of dothiepin, fluoxetine and placebo on psychomotor and actual driving performance." Br J Clin Pharmacol 39(4): 397-404.

Ramaekers, J. G., H. F. Swijgman, et al. (1992) "Effects of moclobemide and mianserin on highway driving, psychometric performance and subjective parameters, relative to placebo." Psychopharmacology, S62-67.

Ramaekers, J. G., M. M. Uiterwijk, et al. (1992) "Effects of loratadine and cetirizine on actual driving and psychometric test performance, and EEG during driving." European journal of clinical pharmacology, 363-369. 
Rapoport, M. J., N. Herrmann, et al. (2008). "Psychotropic medications and motor vehicle collisions in patients with dementia." Journal of the American Geriatrics Society 56(10): 1968-1970.

Rapoport, M. J., G. Naglie, et al. (2013). "The relationship between cognitive performance, perceptions of driving comfort and abilities, and self-reported driving restrictions among healthy older drivers." Accident Analysis \& Prevention 61(0): 288-295.

Rapoport, M. J., B. Zagorski, et al. (2011). "At-Fault Motor Vehicle Crash Risk in Elderly Patients Treated With Antidepressants." American Journal of Geriatric Psychiatry 19(12): 998-1006.

Rappaport, J. (2009). "The increasing importance of quality of life." Journal of economic geography 11(9): 779-804.

Ravera, S., N. van Rein, et al. (2011). "Road traffic accidents and psychotropic medication use in The Netherlands: a case-control study." British Journal Of Clinical Pharmacology 72(3): 505-513.

Ray, W. A., R. L. Fought, et al. (1992). "Psychoactive drugs and the risk of injurious motor vehicle crashes in elderly drivers." American Journal of Epidemiology 136(7): 873-883.

Redelmeier, D. A., A. B. Kenshole, et al. (2009). "Motor vehicle crashes in diabetic patients with tight glycemic control: A population-based case control analysis." PLoS Medicine 6(12).

Reimer, B., L. D'Ambrosio, et al. (2008). "Effects of Age on Spinal Rotation During a Driving Task." Transportation Research Record: Journal of the Transportation Research Board 2078(-1): 57-61.

Ridout, F. and I. Hindmarch (2001) "Effects of tianeptine and mianserin on car driving skills." Psychopharmacology, 356-361.

Ridout, F., R. Meadows, et al. (2003). "A placebo controlled investigation into the effects of paroxetine and mirtazapine on measures related to car driving performance." Human Psychopharmacology 18(4): 261-269.

Ridout, F., Z. Shamsi, et al. (2003). "A single-center, randomized, double-blind, placebo-controlled, crossover investigation of the effects of fexofenadine hydrochloride $180 \mathrm{mg}$ alone and with alcohol, with hydroxyzine hydrochloride $50 \mathrm{mg}$ as a positive internal control, on aspects of cognitive and psychomotor function related to driving a car." Clinical Therapeutics (USA) 25(May): 1518-1538.

Riedel, W. J., R. Quasten, et al. (1988). A Study Comparing the Hypnotic Efficacies and Residual Effects on Actual Driving Performance of Midazolam $15 \mathrm{mg}$, Triazolam $0.5 \mathrm{mg}$, Temazepam $20 \mathrm{mg}$ and Placebo in Shift Workers on Night Duty: 57p.

Rosenbloom, S. (2003). The mobility needs of older americans: implications for transportation reauthorization. T. B. Institution. Washington DC,

Ross, L. A., K. J. Anstey, et al. (2009). "Older drivers in Australia: trends in driving status and cognitive and visual impairment." Journal of the American Geriatrics Society 57(10): 1868-1873. 
Rothman, K. J., S. Greenland, et al. (2008). Modern epidemiology--third edition. Philadelphia, Lippincott Williams and Wilkins,.

Ryan, G. A., M. Legge, et al. (1998). "Age related changes in drivers' crash risk and crash type." Accident Analysis \& Prevention 30(3): 379-387.

Sagberg, F. (2006). "Driver health and crash involvement: A case-control study." Accident Analysis \& Prevention 38(1): 28-34.

Sandlin, D., G. McGwin, Jr., et al. (2014). "Association between vision impairment and driving exposure in older adults aged 70 years and over: a population-based examination." Acta Ophthalmol 92(3): e207-212.

Satariano, W. A., K. E. MacLeod, et al. (2004). "Problems With Vision Associated With Limitations or Avoidance of Driving in Older Populations." The Journals of Gerontology Series B:

Psychological Sciences and Social Sciences 59(5): S281-S286.

Schmidt, U., D. Brendemühl, et al. (1986) "Aspects of driving after hypnotic therapy with particular reference to temazepam." Acta psychiatrica Scandinavica. Supplementum, 112-118.

Schwarzlose, A. A., J. W. Mjelde, et al. (2014). "Willingness to pay for public transportation options for improving quality of life in rural elderly." Transportation Research Part A 61: 1-14.

Signorovitch, J. E., D. Macaulay, et al. (2013). "Hypoglycaemia and accident risk in people with type 2 diabetes mellitus treated with non-insulin antidiabetes drugs." Diabetes Obes Metab 15(4): 335341.

Skurtveit, S., H. Strøm, et al. (2009). "Road traffic accident risk in patients with diabetes mellitus receiving blood glucose-lowering drugs. Prospective follow-up study." Diabetic Medicine 26(4): 404-408.

Spokoyny, I., N. Barazangi, et al. (2014). "Reduced clopidogrel metabolism in a multiethnic population: prevalence and rates of recurrent cerebrovascular events." J Stroke Cerebrovasc Dis 23(4): 694698.

Stein, C. and C. Baerwald (2014). "Opioids for the treatment of arthritis pain." Expert Opin Pharmacother 15(2): 193-202.

Stevenson, G. W., M. N. Pathria, et al. (1986). "Driving ability after intravenous fentanyl or diazepam. A controlled double-blind study." Investigative Radiology 21(9): 717-719.

Stewart, R. B. (1993). "Driving accidents in the elderly: An analysis of symptoms, diseases, and medications." Journal of geriatric drug therapy 8(2): 31-44.

Stoduto, G., R. E. Mann, et al. (2012). "Examining the link between collision involvement and cocaine use." Drug \& Alcohol Dependence 123(1-3): 260-263. 
Stutts, J. (2005). NCHRP Synthesis 348: improving the safety of older road users. Transportation. Washington DC,.

Surapaneni, V. K. (2006). Identification and comparison of crash risk for older drivers, UMI Dissertation Services, ProQuest Information and Learning, Ann Arbor, MI.

Tang, F., E. Choi, et al. (2013). "Older americans employment and retirement." Aging International 38: $82-94$.

Tashiro, M., E. Horikawa, et al. (2005). "Effects of fexofenadine and hydroxyzine on brake reaction time during car-driving with cellular phone use." Human Psychopharmacology: Clinical \& Experimental 20(7): 501-509.

Tavris, D. R., E. M. Kuhn, et al. (2001). "Age and gender patterns in motor vehicle crash injuries: importance of type of crash and occupant role." Accident Analysis and Prevention 33(2): 167172.

Theunissen, E. L., D. Street, et al. (2013). "A randomized trial on the acute and steady-state effects of a new antidepressant, vortioxetine (Lu AA21004), on actual driving and cognition." Clinical Pharmacology And Therapeutics 93(6): 493-501.

Theunissen, E. L., A. Vermeeren, et al. (2006) "Repeated-dose effects of mequitazine, cetirizine and dexchlorpheniramine on driving and psychomotor performance." British journal of clinical pharmacology, 79-86 DOI: 10.1111/j.1365-2125.2005.02524.x.

Theunissen, E. L., A. Vermeeren, et al. (2004). "A dose-ranging study of the effects of mequitazine on actual driving, memory and psychomotor performance as compared to dexchlorpheniramine, cetirizine and placebo." Clinical And Experimental Allergy: Journal Of The British Society For Allergy And Clinical Immunology 34(2): 250-258.

Topiwala, A., S. Patel, et al. (2014). "Health benefits of encore careers for baby boomers." Maturitas 78(1): 8-10.

Tuokko, H., K. Tallman, et al. (1995). "An examination of driving records in a dementia clinic." $\underline{\mathbf{J}}$ Gerontol B Psychol Sci Soc Sci 50(3): S173-181.

Unterecker, S., C. Hiemke, et al. (2012). "The Effect of Age, Sex, Smoking and Co-Medication on Serum Levels of Venlafaxine and O-Desmethylvenlafaxine under Naturalistic Conditions." Pharmacopsychiatry 45(06): 229-235.

Van Laar, M., A. Van Willigenburg, et al. (1993). "Over-the-road and simulated driving: comparison of measures of the hangover effects to two benzodiazepine hypnotics." Proceedings International Council on Alcohol, Drugs and Traffic Safety Conference 1993: 672-677. 
Van Laar, M., E. Volkerts, et al. (2001). "Subchronic effects of the GABA-agonist lorazepam and the 5HT2A/2C antagonist ritanserin on driving performance, slow wave sleep and daytime sleepiness in healthy volunteers." Psychopharmacology (Berl) 154(2): 189-197.

Van Laar, M. W., E. R. Volkerts, et al. (1992). "Therapeutic effects and effects on actual driving performance of chronically administered buspirone and diazepam in anxious outpatients." Journal Of Clinical Psychopharmacology 12(2): 86-95.

Vermeeren, A., P. E. Danjou, et al. (1998). "Residual effects of evening and middle-of-the-night administration of zaleplon 10 and $20 \mathrm{mg}$ on memory and actual driving performance." Human Psychopharmacology: Clinical \& Experimental 13: S98-S107.

Vermeeren, A. and J. F. O'Hanlon (1998) "Fexofenadine's effects, alone and with alcohol, on actual driving and psychomotor performance." Journal of allergy and clinical immunology, 306-311 DOI: 10.1016/S0091-6749(98)70240-4.

Vermeeren, A., J. G. Ramaekers, et al. (2002) "Effects of emedastine and cetirizine, alone and with alcohol, on actual driving of males and females." Journal of psychopharmacology (Oxford, England), 57-64.

Vermeeren, A., J. G. Ramaekers, et al. (1998). "Residual effects on actual car driving of evening dosing of chlorpheniramine 8 and $12 \mathrm{mg}$ when used with terfenadine $60 \mathrm{mg}$ in the morning." Human Psychopharmacology: Clinical \& Experimental 13: S79-S86.

Vermeeren, A., W. J. Riedel, et al. (2002) "Differential residual effects of zaleplon and zopiclone on actual driving: a comparison with a low dose of alcohol." Sleep, 224-231.

Verster, J. C., D. S. Veldhuijzen, et al. (2006). "Effects of an opioid (oxycodone/paracetamol) and an NSAID (bromfenac) on driving ability, memory functioning, psychomotor performance, pupil size, and mood." Clinical Journal of Pain 22(5): 499-504.

Verster, J. C., E. R. Volkerts, et al. (2002). "Residual effects of middle-of-the-night administration of zaleplon and zolpidem on driving ability, memory functions, and psychomotor performance." Journal of Clinical Psychopharmacology 22(6): 576-583.

Verster, J. C., E. R. Volkerts, et al. (2002). "Effects of Alprazolam on Driving Ability, Memory Functioning and Psychomotor Performance: A Randomized, Placebo-controlled Study." Neuropsychopharmacology 27(2): 260.

Verster, J. C., A. M. Weert, et al. (2003) "Driving ability after acute and sub-chronic administration of levocetirizine and diphenhydramine: a randomized, double-blind, placebo-controlled trial." Psychopharmacology, 84-90 DOI: 10.1007/s00213-003-1462-6. 
Volkerts, E. R., M. W. V. Laar, et al. (1992). "A Comparative Study of On-the-road and Simulated Driving Performance after Nocturnal Treatment with Lormetazepam $1 \mathrm{mg}$ and Oxazepam 50 mg." Human Psychopharmacology: Clinical \& Experimental 7(5): 297-309.

Volkerts, E. R., W. Van, et al. (1992) "Does cetirizine belong to the new generation of antihistamines? An investigation into its acute and subchronic effects on highway driving, psychometric test performance and daytime sleepiness." Human psychopharmacology, 227-238.

Vuurman, E., T. Eef, et al. (2007). "Lack of Effects Between Rupatadine 10 Mg and Placebo on Actual Driving Performance of Healthy Volunteers." Human Psychopharmacology: Clinical \& Experimental 22(5): pp 289-297.

Vuurman, E. F., G. H. Rikken, et al. (2004). "Effects of desloratadine, diphenhydramine, and placebo on driving performance and psychomotor performance measurements." Eur J Clin Pharmacol 60(5): 307-313.

Vuurman, E. F., M. M. Uiterwijk, et al. (1994). "Effects of mizolastine and clemastine on actual driving and psychomotor performance in healthy volunteers." European Journal Of Clinical Pharmacology 47(3): 253-259.

Webber, S. C., M. M. Porter, et al. (2010). "Mobility in Older Adults: A Comprehensive Framework." The Gerontologist 50(4): 443-450.

Williams, A. F. and V. I. Shabanova (2003). "Responsibility of drivers, by age and gender, for motorvehicle crash deaths." J Safety Res 34(5): 527-531.

Willumeit, H. P., H. Ott, et al. (1991). "Effects of Lormetazepam and other Benzodiazepine Receptor Ligands on Car Driving-related Skills." Human Psychopharmacology: Clinical \& Experimental 6(3): 209-218

Wingen, M., J. Bothmer, et al. (2005). "Actual driving performance and psychomotor function in healthy subjects after acute and subchronic treatment with escitalopram, mirtazapine, and placebo: a crossover trial." The Journal Of Clinical Psychiatry 66(4): 436-443.

Wingen, M., J. G. Ramaekers, et al. (2006). "Driving impairment in depressed patients receiving longterm antidepressant treatment." Psychopharmacology 188(1): 84-91.

Yang, Y. H., J. N. Lai, et al. (2011). "Increased risk of hospitalization related to motor vehicle accidents among people taking zolpidem: A case-crossover study." Journal of Epidemiology 21(1): 37-43.

\section{Chapter 2}

Amanda, H. and C. David B. (2014). "Medications and impaired driving: A review of the literature." Annals of pharmacotherapy ePub(ePub): ePub-ePub. 
Ammon, S., U. Hofmann, et al. (1999). "Pharmacokinetics of dihydrocodeine and its active metabolite after single and multiple oral dosing." Br J Clin Pharmacol 48(3): 317-322.

Augustijns, P., B. Wuyts, et al. (2014). "A review of drug solubility in human intestinal fluids: implications for the prediction of oral absorption." Eur J Pharm Sci 57: 322-332.

Bachs, L. C., A. Engeland, et al. (2009). "The risk of motor vehicle accidents involving drivers with prescriptions for codeine or tramadol." Clinical Pharmacology and Therapeutics 85(6): 596-599.

Betts, T. A. and J. Birtle (1982). "Effect of two hypnotic drugs on actual driving performance next morning." British Medical Journal (England) 285(Sep 25): 852.

Biehl, B. (1979). "Studies of clobazam and car-driving." British Journal Of Clinical Pharmacology 7

Suppl 1: 85S-90S.

Bocca, M. L., F. Le Doze, et al. (1999). "Residual effect of zolpidem $10 \mathrm{mg}$ and zopiclone $7.5 \mathrm{mg}$ versus flunitrazepam $1 \mathrm{mg}$ and placebo on driving performance and ocular saccades." Psychopharmacology 143(4): 373-379.

Bocca, M. L., S. Marie, et al. (2011) "Zolpidem and zopiclone impair similarly monotonous driving performance after a single nighttime intake in aged subjects." Psychopharmacology, 699-706 DOI: 10.1007/s00213-010-2075-5.

Borgeat, A. (2010). "Do Opioids Affect the Ability to Drive Safely?" Journal of Pain \& Palliative Care Pharmacotherapy 24(2): 167-169.

Bramness, J. G., S. Skurtveit, et al. (2012). "An increased risk of motor vehicle accidents after prescription of methadone." Addiction 107(5): 967-972.

Bramness, J. G., S. Skurtveit, et al. (2007). "The risk of traffic accidents after prescriptions of carisoprodol." Accident Analysis \& Prevention 39(5): pp 1050-1055.

Bramness, J. G., S. Skurtveit, et al. (2009). "An increased risk of road traffic accidents after prescriptions of lithium or valproate?" Pharmacoepidemiology And Drug Safety 18(6): 492-496.

Brookhuis, K. A., E. R. Volkerts, et al. (1990). "Repeated dose effects of lormetazepam and flurazepam upon driving performance." European Journal Of Clinical Pharmacology 39(1): 83-87. 
Brown, L. B. and B. R. Ott (2004). "Driving and dementia: a review of the literature." J Geriatr Psychiatry Neurol 17(4): 232-240.

Brown, T., G. Milavetz, et al. (2013). Alcohol, drugs and driving: Implications for evaluating driver impairment. 57th Annual Scientific Conference of the Association for the Advancement of Automotive Medicine, Quebec City, QC.

Brunnauer, A. and G. Laux (2013). "The effects of most commonly prescribed second generation antidepressants on driving ability: a systematic review : 70th Birthday Prof. Riederer." Journal Of Neural Transmission (Vienna, Austria: 1996) 120(1): 225-232.

Bushardt, R. L., E. B. Massey, et al. (2008). "Polypharmacy: misleading, but manageable." Clin Interv Aging 3(2): 383-389.

Carr, D. B. (2000). "The older adult driver." Am Fam Physician 61(1): 141-146, 148.

Carr, D. B., J. M. Duchek, et al. (2006). "Older adult drivers with cognitive impairment." $\underline{\text { Am Fam }}$ Physician 73(6): 1029-1034.

Cheung, I. and A. T. McCartt (2011). "Declines in fatal crashes of older drivers: changes in crash risk and survivability." Accid Anal Prev 43(3): 666-674.

Cooper, L., L. B. Meuleners, et al. (2011). "Psychotropic medications and crash risk in older drivers: A review of the literature." Asia-Pacific Journal of Public Health 23(4): 443-457.

Coopersmith, H. G., N. A. Korner-Bitensky, et al. (1989). "Determining medical fitness to drive: physicians' responsibilities in Canada." $\underline{\mathrm{CMAJ}}$ 140(4): 375-378.

Corsenac, P., E. Lagarde, et al. (2012). "Road traffic crashes and prescribed methadone and buprenorphine: A french registry-based case-control study." Drug \& Alcohol Dependence 123(13): 91-97.

Coupland, C. A. C., P. Dhiman, et al. (2011). "A study of the safety and harms of antidepressant drugs for older people: A cohort study using a large primary care database." Health Technology Assessment 15(28): 5-218. 
Cross, J. M., G. McGwin, Jr., et al. (2009). "Visual and medical risk factors for motor vehicle collision involvement among older drivers." Br J Ophthalmol 93(3): 400-404.

Cui, X. (2001). Injurious crash risk and medical condition and medication use among senior drivers. NQ60285 Ph.D., University of Alberta (Canada).

Daly, A. K. (2014). "Is there a need to teach pharmacogenetics?" Clin Pharmacol Ther 95(3): 245-247. Dassanayake, T., P. Michie, et al. (2011). "Effects of Benzodiazepines, Antidepressants and Opioids on Driving." Drug Safety 34(2): 125-156.

Daurat, A., P. Sagaspe, et al. (2013). "Lorazepam impairs highway driving performance more than heavy alcohol consumption." Accident Analysis \& Prevention 60: 31-34.

De Gier, J. J. (1984). "Driving tests with patients." British Journal of Clinical Pharmacology (England) 18(Suppl. 1): 103-108.

de Gier, J. J., B. J. t Hart, et al. (1981). "Psychomotor performance and real driving performance of outpatients receiving diazepam." Psychopharmacology 73(4): 340-344.

Deits, C., L. Boyle, et al. (2011). Driving Performance of Drug-Impaired Bus Drivers in Work Zone Areas.

Delaney, J. A. C., L. Opatrny, et al. (2006). "Warfarin use and the risk of motor vehicle crash in older drivers." British Journal of Clinical Pharmacology 61(2): 229-232.

Downs, S. H. and N. Black (1998). "The feasibility of creating a checklist for the assessment of the methodological quality both of randomised and non-randomised studies of health care interventions." J Epidemiol Community Health 52(6): 377-384.

Ellen, R. L., S. C. Marshall, et al. (2006). "Systematic review of motor vehicle crash risk in persons with sleep apnea." J Clin Sleep Med 2(2): 193-200.

Elvik, R. (2013). "Risk of road accident associated with the use of drugs: A systematic review and metaanalysis of evidence from epidemiological studies." Accident Analysis \& Prevention 60: 254-267. 
Etminan, M., B. Hemmelgarn, et al. (2004). "Use of lithium and the risk of injurious motor vehicle crash in elderly adults: case-control study nested within a cohort." BMJ: British Medical Journal (International Edition) 328(7439): 558-559.

Fishbain, D. A., R. B. Cutler, et al. (2002). "Can patients taking opioids drive safely? A structured evidence-based review." J Pain Palliat Care Pharmacother 16(1): 9-28.

Fishbain, D. A., R. B. Cutler, et al. (2003). "Are Opioid-Dependent/Tolerant Patients Impaired in Driving-Related Skills? A Structured Evidence-Based Review." Journal of Pain \& Symptom Management 25(6): 559.

Foley, D. J., R. B. Wallace, et al. (1995). "Risk factors for motor vehicle crashes among older drivers in a rural community." J Am Geriatr Soc 43(7): 776-781.

Friedel, B., S. Joo, et al. (1991). "TESTING DRIVERS TAKING DIAZEPAM IN THE DAIMLERBENZ DRIVING SIMULATOR." Journal of traffic medicine 19(1.2): p. 15-27.

Gibson, J. E., R. B. Hubbard, et al. (2009). "Use of Self-controlled Analytical Techniques to Assess the Association Between Use of Prescription Medications and the Risk of Motor Vehicle Crashes." American Journal of Epidemiology 169(6): 761-768.

Gjerde, H., A. S. Christophersen, et al. (2013). "Associations between substance use among car and van drivers in Norway and fatal injury in road traffic accidents: A case-control study." Transportation Research: Part F 17: 134-144.

Gjerde, H., P. T. Normann, et al. (2011). "Alcohol, psychoactive drugs and fatal road traffic accidents in Norway: A case-control study." Accident Analysis \& Prevention 43(3): pp 1197-1203.

Gunja, N. (2013). "In the Zzz zone: the effects of Z-drugs on human performance and driving." Journal Of Medical Toxicology: Official Journal Of The American College Of Medical Toxicology 9(2): $163-171$.

Gustavsen, I., J. G. Bramness, et al. (2008). "Road traffic accident risk related to prescriptions of the hypnotics zopiclone, zolpidem, flunitrazepam and nitrazepam." Sleep Medicine 9(8): 818-822. 
Hansen, R. N. (2012). Investigating the Association Between Sedative Hypnotic Prescription Exposures and Motor Vehicle Crashes. 3521668 Ph.D., University of Washington.

Hansotia, P. and S. K. Broste (1991). "The effect of epilepsy or diabetes mellitus on the risk of automobile accidents." N Engl J Med 324(1): 22-26.

Hayman, A. V. and M. L. Crandall (2009). "Deadly partners: interdependence of alcohol and trauma in the clinical setting." Int J Environ Res Public Health 6(12): 3097-3104.

Hemmelgarn, B., L. E. Lévesque, et al. (2006). "Anti-diabetic drug use and the risk of motor vehicle crash in the elderly." Canadian Journal of Clinical Pharmacology 13(1): e112-e120.

Hindmarch, I. and A. C. Gudgeon (1980). "The effects of clobazam and lorazepam on aspects of psychomotor performance and car handling ability." British Journal Of Clinical Pharmacology 10(2): $145-150$.

Huisingh, C., G. McGwin, Jr., et al. (2013). "Frequent Falling and Motor Vehicle Collision Involvement of Older Drivers." J Am Geriatr Soc.

IMS Health. (2014). "U.S. pharmaceutical sales-Q4 2013." Retrieved 10/1/2014, from http://www.drugs.com/stats/top100/sales.

Irving, A. and W. Jones (1992). "Methods for testing impairment of driving due to drugs." European Journal Of Clinical Pharmacology 43(1): 61-66.

Jones, J. D., S. Mogali, et al. (2012). "Polydrug abuse: a review of opioid and benzodiazepine combination use." Drug Alcohol Depend 125(1-2): 8-18.

Joris C., V. and M. Monique Anna Johanna (2009). "Psychoactive medication and traffic safety." International Journal of Environmental Research and Public Health 6(3): 1041-1054.

Jusko, W. J. (2013). "Moving from basic toward systems pharmacodynamic models." J Pharm Sci 102(9): 2930-2940.

Kelly, E., S. Darke, et al. (2004). "A review of drug use and driving: epidemiology, impairment, risk factors and risk perceptions." Drug \& Alcohol Review 23(3): 319-344. 
Kelly, R., T. Warke, et al. (1999). "Medical restrictions to driving: the awareness of patients and doctors." Postgrad Med J 75(887): 537-539.

Koepsell, T. D., M. E. Wolf, et al. (1994). "Medical conditions and motor vehicle collision injuries in older adults." Journal of the American Geriatrics Society 42(7): 695-700.

Kress, H. G. and B. Kraft (2005). "Opioid medication and driving ability." European Journal Of Pain (London, England) 9(2): 141-144.

Krueger, G. P. (2010). "Psychoactive Medications, Stimulants, Hypnotics, and Nutritional Aids: Effects on Driving Alertness and Performance." Journal of the Washington Academy of Sciences 96(3): pp 51-85.

Kuitunen, T. (1994). "Drug and ethanol effects on the clinical test for drunkenness: single doses of ethanol, hypnotic drugs and antidepressant drugs." Pharmacology \& Toxicology 75(2): 91-98.

Laurell, H. and J. Tornros (1986). "The carry-over effects of triazolam compared with nitrazepam and placebo in acute emergency driving situations and in monotonous simulated driving." Acta Pharmacol Toxicol (Copenh) 58(3): 182-186.

Lenné, M. G., P. Dietze, et al. (2003) "The effects of the opioid pharmacotherapies methadone, LAAM and buprenorphine, alone and in combination with alcohol, on simulated driving." Drug and alcohol dependence, $271-278$.

Leufkens, T. R. M., J. S. Lund, et al. (2009). "Highway driving performance and cognitive functioning the morning after bedtime and middle-of-the-night use of gaboxadol, zopiclone and zolpidem." Journal Of Sleep Research 18(4): 387-396.

Leufkens, T. R. M. and A. Vermeeren (2009). "Highway driving in the elderly the morning after bedtime use of hypnotics: A comparison between temazepam $20 \mathrm{mg}$, zopiclone $7.5 \mathrm{mg}$, and placebo." Journal of Clinical Psychopharmacology 29(5): 432-438.

Leufkens, T. R. M. and A. Vermeeren (2014). "Zopiclone's Residual Effects on Actual Driving Performance in a Standardized Test: A Pooled Analysis of Age and Sex Effects in 4 PlaceboControlled Studies." Clinical Therapeutics 36(1): 141-150. 
Leung, S., R. Chapman, et al. (2013). Medicinal drug use and driving.

Leung, S. Y. (2011). "Benzodiazepines, opioids and driving: An overview of the experimental research." Drug \& Alcohol Review 30(3): 281-286.

Linnoila, M. and S. Hakkinen (1974). "Effects of diazepam and codeine, alone and in combination with alcohol, on simulated driving." Clin Pharmacol Ther 15(4): 368-373.

Lonnen, K. F., R. J. Powell, et al. (2008). "Road traffic accidents and diabetes: insulin use does not determine risk." Diabetic Medicine 25(5): 578-584.

Mailis-Gagnon, A., S. F. Lakha, et al. (2012). "Systematic review of the quality and generalizability of studies on the effects of opioids on driving and cognitive/psychomotor performance." Clinical Journal of Pain 28(6): 542-555.

Marottoli, R. A., A. M. Ostfeld, et al. (1993). "Driving cessation and changes in mileage driven among elderly individuals." J Gerontol 48(5): S255-260.

Martinez, R. (1995). "Older drivers and physicians." JAMA 274(13): 1060.

Mathijssen, M. P. M. (2006). The risk of drink and drug driving--results of a case-control study conducted in the Netherlands.

Mattila, M. J., T. Kuitunen, et al. (1993). "RELATED COORDINATIVE, REACTIVE AND COGNITIVE PERFORMANCES AS IMPAIRED BY DRUGS AND ALCOHOL: COMPARISON WITH CLINICAL TEST FOR DRIVING FITNESS." Journal of traffic medicine 21(3): p. 101-114.

McGwin, G., Jr., R. V. Sims, et al. (2000). "Relations among chronic medical conditions, medications, and automobile crashes in the elderly: a population-based case-control study." Am J Epidemiol 152(5): 424-431.

Mercier-Guyon, C., J. Lejay, et al. (1999). "Comparative Study of the Effects of Captodiamine and Lorazepam on Car Driving Ability." Clinical Drug Investigation 17(6): 451-459.

Meskali, M., C. Berthelon, et al. (2009). "Residual effects of hypnotic drugs in aging drivers submitted to simulated accident scenarios: An exploratory study." Psychopharmacology 207(3): 461-467. 
Mets, M. A., J. M. Vries, et al. (2011) "Next-day effects of ramelteon (8 mg), zopiclone (7.5 mg), and placebo on highway driving performance, memory functioning, psychomotor performance, and mood in healthy adult subjects." Sleep, 1327-1334 DOI: 10.5665/SLEEP.1272.

Mintzer, M. Z., R. K. Lanier, et al. (2010). "Effects of repeated tramadol and morphine administration on psychomotor and cognitive performance in opioid-dependent volunteers." Drug Alcohol Depend 111(3): 265-268.

Morland, J. (2000). "Driving under the influence of non-alcohol drugs." Forensic Sci.Rev 12: 80-105.

Morrison, J. B., L. N. Boyle, et al. (2011). PATH - Post-Accident Testing Heuristics: 217p.

Moskowitz, H. and A. Smiley (1982) "Effects of chronically administered buspirone and diazepam on driving-related skills performance." Journal of clinical psychiatry, 45-55.

Moták, L., L. Bayssac, et al. (2014). "Naturalistic conversation improves daytime motorway driving performance under a benzodiazepine: A randomised, crossover, double-blind, placebo-controlled study." Accident Analysis and Prevention 67: 61-66.

Movig, K. L. L., M. P. M. Mathijssen, et al. (2004). "Psychoactive substance use and the risk of motor vehicle accidents." Accident Analysis \& Prevention 36(4): 631.

Mura, P., P. Kintz, et al. (2003). "Comparison of the prevalence of alcohol, cannabis and other drugs between 900 injured drivers and 900 control subjects: Results of a French collaborative study." Forensic Science International 133(1-2): 79-85.

National Highway Traffic Safety Administration (2010). Drug Involvement of Fatally Injured Drivers. Traffic Safety Facts United States Department of Transportation. November: 1-3.

Nelson, R. C. (1992). Psychotherapeutic drugs, mental disorders and automobile crashes: A case control study of 1308 females. (Volumes I and II). 9222476 Ph.D., University of Minnesota.

Neutel, I. (1998). "Benzodiazepine-related traffic accidents in young and elderly drivers." Human Psychopharmacology: Clinical \& Experimental 13: S115-S123. 
Nilsen, H. K., N. I. Landr, et al. (2011). "Driving Functions in a Video Simulator in Chronic Nonmalignant Pain Patients Using and Not Using Codeine." European Journal of Pain 15(4): pp 409415.

O'Hanlon, J. F., T. W. Haak, et al. (1982). "Diazepam impairs lateral position control in highway driving." Science 217(4554): 79-81.

O'Hanlon, J. F., A. Vermeeren, et al. (1995). "Anxiolytics' effects on the actual driving performance of patients and healthy volunteers in a standardized test. An integration of three studies." Neuropsychobiology 31(2): 81-88.

O'Hanlon, J. F. and E. R. Volkerts (1986). "Hypnotics and actual driving performance." Acta Psychiatrica Scandinavica. Supplementum 332: 95-104.

Orriols, L., P. Philip, et al. (2011). "Benzodiazepine-like hypnotics and the associated risk of road traffic accidents." Clinical Pharmacology and Therapeutics 89(4): 595-601.

Orriols, L., L. R. Salmi, et al. (2009). "The impact of medicinal drugs on traffic safety: a systematic review of epidemiological studies." Pharmacoepidemiol Drug Saf 18(8): 647-658.

Oster, C. V. and J. Strong (2013). "Analyzing road safety in the United States." Research in Transportation Economics 43: 98-111.

Otmani, S., A. Demazières, et al. (2008) "Effects of prolonged-release melatonin, zolpidem, and their combination on psychomotor functions, memory recall, and driving skills in healthy middle aged and elderly volunteers." Human psychopharmacology, 693-705 DOI: 10.1002/hup.980.

Partinen, M., K. Hirvonen, et al. (2003). "Effects of after-midnight intake of zolpidem and temazepam on driving ability in women with non-organic insomnia." Sleep Medicine 4(6): 553.

Popescu, F. D. (2008). "H1 antihistamines and driving." Journal of medicine and life 1(3): 262-268.

Purgato, M. and C. E. Adams (2012). "Heterogeneity: the issue of apples, oranges and fruit pie." Epidemiol Psychiatr Sci 21(1): 27-29.

Ramaekers, J. G. (2003). "Antidepressants and driver impairment: empirical evidence from a standard onthe-road test." J Clin Psychiatry 64(1): 20-29. 
Rapoport, M. J. and M. C. Baniña (2007). "Impact of Psychotropic Medications on Simulated Driving: A Critical Review." CNS Drugs 21(6): 503.

Rapoport, M. J., K. L. Lanctôt, et al. (2009). "Benzodiazepine use and driving: A meta-analysis." Journal of Clinical Psychiatry 70(5): 663-673.

Ravera, S., J. G. Ramaekers, et al. (2012). "Are Selective Serotonin Reuptake Inhibitors Safe for Drivers? What is the Evidence?" Clinical Therapeutics 34(5): 1070-1083.

Riedel, W. J. (1991). "EYE-MOVEMENTS, EXPERT RATINGS, WEAVING AND TIME-TO-LINECROSSING AS MEASURES OF DRIVING PERFORMANCE AND DRIVING PERFORMANCE IMPAIRMENT." VISION IN VEHICLES--III / EDITED BY A.G. GALE; CO-EDITED BY I. D. BROWN... ET AL.. --.

Riedel, W. J., R. Quasten, et al. (1988). A Study Comparing the Hypnotic Efficacies and Residual Effects on Actual Driving Performance of Midazolam 15 mg, Triazolam 0.5 mg, Temazepam 20 mg and Placebo in Shift Workers on Night Duty: 57p.

Roberts, D. J. (2005). "Antihistamines and driving ability: evidence from on-the-road driving studies during normal traffic." Ann Allergy Asthma Immunol 94(3): 407-409; author reply 409-410.

Rockett, I. R., M. D. Regier, et al. (2012). "Leading causes of unintentional and intentional injury mortality: United States, 2000-2009." Am J Public Health 102(11): e84-92.

Rothman, K. J., S. Greenland, et al. (2008). Modern Epidemiology. Philadelphia, Lippincott Williams \& Williams,

Sagberg, F. (2006). "Driver health and crash involvement: a case-control study." Accid Anal Prev 38(1): 28-34.

Schmidt, U., D. Brendemühl, et al. (1986) "Aspects of driving after hypnotic therapy with particular reference to temazepam." Acta psychiatrica Scandinavica. Supplementum, 112-118.

Sims, R. V., G. McGwin, Jr., et al. (2000). "Exploratory study of incident vehicle crashes among older drivers." Journals of Gerontology: Series A: Biological Sciences and Medical Sciences 55A(1): M22-M27. 
Sise, R. G., R. Y. Calvo, et al. (2014). "The epidemiology of trauma-related mortality in the United States from 2002 to 2010." J Trauma Acute Care Surg 76(4): 913-919; discussion 920.

Skurtveit, S., H. Strøm, et al. (2009). "Road traffic accident risk in patients with diabetes mellitus receiving blood glucose-lowering drugs. Prospective follow-up study." Diabetic Medicine 26(4): 404-408.

Smiley, A. and H. Moskowitz (1986). "Effects of long-term administration of buspirone and diazepam on driver steering control." Am J Med 80(3B): 22-29.

Smink, B. E., A. Egberts, et al. (2010). "The Relationship between Benzodiazepine Use and Traffic Accidents: A Systematic Literature Review." CNS Drugs 24(8): pp 639-653.

Soyka, M. (2014). "Opioids and Traffic Safety - Focus on Buprenorphine." Pharmacopsychiatry 47(1): 717.

Staner, L., S. Ertlé, et al. (2005) "Next-day residual effects of hypnotics in DSM-IV primary insomnia: a driving simulator study with simultaneous electroencephalogram monitoring." Psychopharmacology, 790-798 DOI: 10.1007/s00213-005-0082-8.

Stein, C. and C. Baerwald (2014). "Opioids for the treatment of arthritis pain." Expert Opin Pharmacother 15(2): 193-202.

Strand, M. C., B. Fjeld, et al. (2013). "Can patients receiving opioid maintenance therapy safely drive? A systematic review of epidemiological and experimental studies on driving ability with a focus on concomitant methadone or buprenorphine administration." Traffic Injury Prevention 14(1): 26-38.

Takahashi, M., K. Iwamoto, et al. (2010) "The effects of acute treatment with tandospirone, diazepam, and placebo on driving performance and cognitive function in healthy volunteers." Human psychopharmacology, 260-267 DOI: 10.1002/hup.1105.

Tamaji, A., K. Iwamoto, et al. (2012) "Differential effects of diazepam, tandospirone, and paroxetine on plasma brain-derived neurotrophic factor level under mental stress." Human psychopharmacology, 329-333 DOI: 10.1002/hup.2220. 
Uc, E. Y., M. Rizzo, et al. (2006). "Driving with distraction in Parkinson disease." Neurology 67(10): 1774-1780.

van Laar, M., E. Volkerts, et al. (2001). "Subchronic effects of the GABA-agonist lorazepam and the 5HT2A/2C antagonist ritanserin on driving performance, slow wave sleep and daytime sleepiness in healthy volunteers." Psychopharmacology (Berl) 154(2): 189-197.

van Laar, M. W. and E. R. Volkerts (1998). "Driving and Benzodiazepine Use: Evidence That They Do Not Mix." CNS Drugs 10(5): 383-396.

van Laar, M. W., E. R. Volkerts, et al. (1992). "Therapeutic effects and effects on actual driving performance of chronically administered buspirone and diazepam in anxious outpatients." Journal Of Clinical Psychopharmacology 12(2): 86-95.

Vanakoski, J., M. J. Mattila, et al. (2000) "Driving under light and dark conditions: effects of alcohol and diazepam in young and older subjects." European journal of clinical pharmacology, 453-458.

Vermeeren, A., T. R. M. Leufkens, et al. (2009). "Effects of Anxiolytics on Driving." pp 289-305.

Vermeeren, A., W. J. Riedel, et al. (2002) "Differential residual effects of zaleplon and zopiclone on actual driving: a comparison with a low dose of alcohol." Sleep, 224-231.

Verster, J., A. Bervoets, et al. (2014). "Lapses of attention as outcome measure of the on-the-road driving test." Psychopharmacology 231(1): 283-292.

Verster, J. C. and M. A. J. Mets (2009). "Psychoactive medication and traffic safety." International Journal Of Environmental Research And Public Health 6(3): 1041-1054.

Verster, J. C. and J. G. Ramaekers (2009). "Antidepressants and Traffic Safety." pp 307-313.

Verster, J. C., D. S. Veldhuijzen, et al. (2006). "Hypnotics and driving safety: meta-analyses of randomized controlled trials applying the on-the-road driving test." Current Drug Safety 1(1): 6371.

Verster, J. C., D. S. Veldhuijzen, et al. (2005). "Is it Safe to Drive a Car when Treated with Anxiolytics? Evidence from onthe- Road Driving Studies During Normal Traffic." Current Psychiatry Reviews 1(2): 215-225. 
Verster, J. C., E. R. Volkerts, et al. (2007). "Zolpidem and Traffic Safety - The Importance of Treatment Compliance." Current Drug Safety 2(3): 220-226.

Verster, J. C., E. R. Volkerts, et al. (2002). "Residual effects of middle-of-the-night administration of zaleplon and zolpidem on driving ability, memory functions, and psychomotor performance." Journal of Clinical Psychopharmacology 22(6): 576-583.

Verster, J. C., E. R. Volkerts, et al. (2007). "Effects of Sleep Medications on Cognition, Psychomotor Skills, Memory and Driving Performance in the Elderly." Current Psychiatry Reviews 3(4): 281292.

Verster, J. C., A. M. Weert, et al. (2003) "Driving ability after acute and sub-chronic administration of levocetirizine and diphenhydramine: a randomized, double-blind, placebo-controlled trial." Psychopharmacology, 84-90 DOI: 10.1007/s00213-003-1462-6.

Walsh, J. M., J. J. de Gier, et al. (2004). "Drugs and driving." Traffic Inj Prev 5(3): 241-253.

Willumeit, H. P., W. Neubert, et al. (1983). "Driving ability following the subchronic application of lormetazepam, flurazepam and placebo." Ergonomics 26(11): 1055-1061.

Willumeit, H. P., H. Ott, et al. (1984). "Simulated car driving as a useful technique for the determination of residual effects and alcohol interaction after short- and long-acting benzodiazepines." Psychopharmacology. Supplementum 1: 182-192.

Willumeit, H. P., H. Ott, et al. (1984). "Alcohol interaction of lormetazepam, mepindolol sulphate and diazepam measured by performance on the driving simulator." Pharmacopsychiatry 17(2): 36-43. Wilson, F. A., J. P. Stimpson, et al. (2014). "Fatal crashes from drivers testing positive for drugs in the U.S., 1993-2010." Public Health Rep 129(4): 342-350.

Yang, Y. H., J. N. Lai, et al. (2011). "Increased risk of hospitalization related to motor vehicle accidents among people taking zolpidem: A case-crossover study." Journal of Epidemiology 21(1): 37-43.

Zacny, J. P., J. A. Paice, et al. (2011). "Characterizing the subjective and psychomotor effects of carisoprodol in healthy volunteers." Pharmacol Biochem Behav 100(1): 138-143. 
Zacny, J. P., J. A. Paice, et al. (2012). "Subjective and psychomotor effects of carisoprodol in combination with oxycodone in healthy volunteers." Drug Alcohol Depend 120(1-3): 229-232.

\section{Chapter 3}

Adis International (2002). "Oxycodone/ibuprofen." Drugs R D 3(1): 56-57.

Bachs, L. C., A. Engeland, et al. (2009). The risk of motor vehicle accidents involving drivers with prescriptions for codeine or tramadol. Clin Pharmacol Ther. United States. 85: 596-599.

Bailey, J. E., P. L. Barton, et al. (2006). "The effect of FDA approval of a generic competitor to OxyContin (oxycodone $\mathrm{HCl}$ controlled-release) tablets on the abuse of oxycodone." Drug Alcohol Depend 84(2): 182-187.

Ball, K., C. Owsley, et al. (1998). "Driving avoidance and functional impairment in older drivers." Accid Anal Prev 30(3): 313-322.

Blanchard, R. A., A. M. Myers, et al. (2010). "Correspondence between self-reported and objective measures of driving exposure and patterns in older drivers." Accid Anal Prev 42(2): 523-529.

Brady, J. E. and G. Li (2013). "Prevalence of alcohol and other drugs in fatally injured drivers." Addiction 108(1): 104-114.

Braver, E. R. and R. E. Trempel (2004). "Are older drivers actually at higher risk of involvement in collisions resulting in deaths or non-fatal injuries among their passengers and other road users?" Inj Prev 10(1): 27-32.

Brown, L. B. and B. R. Ott (2004). "Driving and dementia: a review of the literature." J Geriatr Psychiatry Neurol 17(4): 232-240.

Brown, T., G. Milavetz, et al. (2013). Alcohol, drugs and driving: Implications for evaluating driver impairment. 57th Annual Scientific Conference of the Association for the Advancement of Automotive Medicine. Quebec City, QC.

Bushardt, R. L., E. B. Massey, et al. (2008). "Polypharmacy: misleading, but manageable." Clin Interv Aging 3(2): 383-389.

Carr, D. B. (2000). "The older adult driver." Am Fam Physician 61(1): 141-146, 148. 
Carr, D. B., J. M. Duchek, et al. (2006). "Older adult drivers with cognitive impairment." Am Fam Physician 73(6): 1029-1034.

Centers for Disease Control and Prevention (2006). "Alcohol and other drug use among victims of motorvehicle crashes--West Virginia, 2004-2005." MMWR Morb Mortal Wkly Rep 55(48): 12931296.

Centers for Disease Control and Prevention (2010). "Emergency department visits involving nonmedical use of selected prescription drugs - United States, 2004-2008." MMWR Morb Mortal Wkly Rep 59(23): 705-709.

Centers for Disease Control and Prevention (2011). "Vital signs: overdoses of prescription opioid pain relievers---United States, 1999--2008." MMWR Morb Mortal Wkly Rep 60(43): 1487-1492.

Centers for Disease Control and Prevention. (2015). "Impaired driving." Retrieved 2/16/2015, 2015, from http://www.cdc.gov/motorvehiclesafety/impaired_driving/index.html.

Cheung, I. and A. T. McCartt (2011). "Declines in fatal crashes of older drivers: changes in crash risk and survivability." Accid Anal Prev 43(3): 666-674.

Christensen, L. Q., L. M. Nielsen, et al. (1990). "Traffic accidents and drivers suspected for drug influence." Forensic Sci Int 45(3): 273-280.

Coben, J. H., S. M. Davis, et al. (2010). "Hospitalizations for poisoning by prescription opioids, sedatives, and tranquilizers." Am J Prev Med 38(5): 517-524.

Colliver, J. D., W. M. Compton, et al. (2006). "Projecting drug use among aging baby boomers in 2020." Ann Epidemiol 16(4): 257-265.

Coopersmith, H. G., N. A. Korner-Bitensky, et al. (1989). "Determining medical fitness to drive: physicians' responsibilities in Canada." CMAJ 140(4): 375-378.

Cross, J. M., G. McGwin, Jr., et al. (2009). "Visual and medical risk factors for motor vehicle collision involvement among older drivers." Br J Ophthalmol 93(3): 400-404.

Daly, A. K. (2014). "Is there a need to teach pharmacogenetics?" Clin Pharmacol Ther 95(3): 245-247. 
Ellen, R. L., S. C. Marshall, et al. (2006). "Systematic review of motor vehicle crash risk in persons with sleep apnea." J Clin Sleep Med 2(2): 193-200.

Foley, D. J., H. K. Heimovitz, et al. (2002). "Driving life expectancy of persons aged 70 years and older in the United States." Am J Public Health 92(8): 1284-1289.

Fulton, M. M. and E. R. Allen (2005). "Polypharmacy in the elderly: a literature review." J Am Acad Nurse Pract 17(4): 123-132.

Hansotia, P. and S. K. Broste (1991). "The effect of epilepsy or diabetes mellitus on the risk of automobile accidents." N Engl J Med 324(1): 22-26.

Higgins, J. P., S. W. Wright, et al. (1996). "Alcohol, the elderly, and motor vehicle crashes." Am J Emerg Med 14(3): 265-267.

Hours, M., E. Fort, et al. (2008). "Diseases, consumption of medicines and responsibility for a road crash: a case-control study." Accid Anal Prev 40(5): 1789-1796.

Hughes, C. M. (2004). "Medication non-adherence in the elderly: how big is the problem?" Drugs Aging 21(12): 793-811.

Jones, J. D., S. Mogali, et al. (2012). "Polydrug abuse: a review of opioid and benzodiazepine combination use." Drug Alcohol Depend 125(1-2): 8-18.

Joranson, D. E., K. M. Ryan, et al. (2000). "Trends in medical use and abuse of opioid analgesics." $\underline{\text { JAMA }}$ 283(13): 1710-1714.

Jorgensen, T., S. Johansson, et al. (2001). "Prescription drug use, diagnoses, and healthcare utilization among the elderly." Ann Pharmacother 35(9): 1004-1009.

Kelly, E., S. Darke, et al. (2004). "A review of drug use and driving: epidemiology, impairment, risk factors and risk perceptions." Drug Alcohol Rev 23(3): 319-344.

Kelly, R., T. Warke, et al. (1999). "Medical restrictions to driving: the awareness of patients and doctors." Postgrad Med J 75(887): 537-539.

Lotfipour, S. and F. Vaca (2007). "Commentary: Polypharmacy and older drivers: beyond the doors of the emergency department (ED) for patient safety." Ann Emerg Med 49(4): 535-537. 
Manchikanti, L. (2007). "National drug control policy and prescription drug abuse: facts and fallacies." Pain Physician 10(3): 399-424.

Marottoli, R. A., A. M. Ostfeld, et al. (1993). "Driving cessation and changes in mileage driven among elderly individuals." J Gerontol 48(5): S255-260.

Martinez, R. (1995). "Older drivers and physicians." JAMA 274(13): 1060.

McGwin, G., Jr., R. V. Sims, et al. (2000). "Relations among chronic medical conditions, medications, and automobile crashes in the elderly: a population-based case-control study." Am J Epidemiol 152(5): 424-431.

National Highway Traffic Safety Administration (2010). Drug Involvement of Fatally Injured Drivers. Traffic Safety Facts November: $1-3$.

National Highway Traffic Safety Administration (2012). Fatality Analysis Reporting System Analytical Users Manual 1975-2011.

National Highway Traffic Safety Administration. (2015). "Fact sheet: national roadside survey of alcohol and druguse by drives." Retrieved 2/16/2015, 2015, from http://www.nhtsa.gov/Driving+Safety/Research+\&+Evaluation/Alcohol+and+Drug+Use+By+Dri vers.

Paulozzi, L. J., M. F. Ballesteros, et al. (2006). "Recent trends in mortality from unintentional injury in the United States." J Safety Res 37(3): 277-283.

Pletcher, M. J., S. G. Kertesz, et al. (2008). "Trends in opioid prescribing by race/ethnicity for patients seeking care in US emergency departments." JAMA 299(1): 70-78.

Rubin, D. B., J. L. Schafer, et al. (1998). Multiple imputation of missing blood alcohol concentration values in FARS. Transportation. Wasington, DC, National Highway Traffic Safety Administration: 34 .

Rudisill, T. M., S. Zhao, et al. (2014). "Trends in drug use among drivers killed in U.S. traffic crashes, 1999-2010." Accid Anal Prev 70: 178-187. 
Sagberg, F. (2006). "Driver health and crash involvement: a case-control study." Accid Anal Prev 38(1): $28-34$

SAS Institute (2010). Cary, NC.

Srihari, Y., S. Padmaja, et al. (2009). "Implications of Drug Price Competition and Patent Term Restoration Act (DPCPTRA) on Indian Pharma Industry." Journal of Intellectual Property Rights 14(6): 501-512.

Stein, C. and C. Baerwald (2014). "Opioids for the treatment of arthritis pain." Expert Opin Pharmacother 15(2): 193-202.

Stewart, R. B., M. T. Moore, et al. (1993). "Driving cessation in the elderly: An analysis of symptoms, diseases, and medications." Journal of Geriatric Drug Therapy.

Subramanian, R. (2002). Transitioning to multiple imputation-a new method to estimate missing blood alcohol concentration (BAC) values in FARS. Transportation. Washington, DC, National Highway Traffic Safey Administration: 36.

Uc, E. Y., M. Rizzo, et al. (2006). "Driving with distraction in Parkinson disease." Neurology 67(10): 1774-1780

Verster, J. C. and E. R. Volkerts (2004). "Antihistamines and driving ability: evidence from on-the-road driving studies during normal traffic." Ann Allergy Asthma Immunol 92(3): 294-303; quiz 303295,355

Walsh, J. M., J. J. de Gier, et al. (2004). "Drugs and driving." Traffic Inj Prev 5(3): 241-253.

Wilson, F. A., J. P. Stimpson, et al. (2014). "Fatal crashes from drivers testing positive for drugs in the U.S., 1993-2010." Public Health Rep 129(4): 342-350.

Zou, G. (2004). "A modified poisson regression approach to prospective studies with binary data." Am J Epidemiol 159(7): 702-706.

\section{Chapter 4}


American Medical Association (2010). Physician's guide to assessing and counseling older drivers-second edition,. Chicago.

Augustijns, P., B. Wuyts, et al. (2014). "A review of drug solubility in human intestinal fluids: implications for the prediction of oral absorption." Eur J Pharm Sci 57: 322-332.

Awadzi, K. D., S. Classen, et al. (2008). "Predictors of injury among younger and older adults in fatal motor vehicle crashes." Accid Anal Prev 40(6): 1804-1810.

Bachs, L. C., A. Engeland, et al. (2009). "The risk of motor vehicle accidents involving drivers with prescriptions for codeine or tramadol." Clinical Pharmacology and Therapeutics 85(6): 596-599.

Barbone, F., A. D. McMahon, et al. (1998). Association of road-traffic accidents with benzodiazepine use. Lancet. England. 352: 1331-1336.

Berta, E., M. Harangi, et al. (2014). "Effect of thyroid hormone status and concomitant medication on statin induced adverse effects in hyperlipidemic patients." Pharmazie 69(6): 420-423.

Blanchard, R. A., A. M. Myers, et al. (2010). "Correspondence between self-reported and objective measures of driving exposure and patterns in older drivers." Accid Anal Prev 42(2): 523-529.

Bowman, S. M. and M. E. Aitken (2011). "Assessing external cause of injury coding accuracy for transport injury hospitalizations." Perspect Health Inf Manag 8: 1c.

Brown, L. B. and B. R. Ott (2004). "Driving and dementia: a review of the literature." J Geriatr Psychiatry Neurol 17(4): 232-240.

Brown, T., G. Milavetz, et al. (2013). Alcohol, drugs and driving: Implications for evaluating driver impairment. 57th Annual Scientific Conference of the Association for the Advancement of Automotive Medicine, Quebec City, QC.

Brzaković, B. B., S. D. Vezmar Kovačević, et al. (2012). "Impact of age, weight and concomitant treatment on lamotrigine pharmacokinetics." Journal of Clinical Pharmacy \& Therapeutics 37(6): 693-697.

Bushardt, R. L., E. B. Massey, et al. (2008). "Polypharmacy: misleading, but manageable." $\underline{\text { Clin Interv }}$ Aging 3(2): 383-389. 
Centers for Disease Control and Prevention (2006). "Alcohol and other drug use among victims of motorvehicle crashes--West Virginia, 2004-2005." MMWR Morb Mortal Wkly Rep 55(48): 12931296.

Chang, C. M., E. C. Wu, et al. (2013). "Psychotropic drugs and risk of motor vehicle accidents: a population-based case-control study." Br J Clin Pharmacol 75(4): 1125-1133.

Chu, T. (2014). "Gender differences in pharmakokinectics." U.S. Pharmacist 39(9): 40-43.

Cingi, C., S. Z. Toros, et al. (2013). "Effect of grapefruit juice on bioavailability of montelukast." The Laryngoscope 123(4): 816-819.

Colliver, J. D., W. M. Compton, et al. (2006). "Projecting drug use among aging baby boomers in 2020." Ann Epidemiol 16(4): 257-265.

Coupland, C., P. Dhiman, et al. (2011). "Antidepressant use and risk of adverse outcomes in older people: population based cohort study." BMJ 343: d4551.

Cross, J. M., G. McGwin, Jr., et al. (2009). "Visual and medical risk factors for motor vehicle collision involvement among older drivers." Br J Ophthalmol 93(3): 400-404.

Cui, X. (2001). Injurious crash risk and medical condition and medication use among senior drivers. NQ60285 Ph.D., University of Alberta (Canada).

Daly, A. K. (2014). "Is there a need to teach pharmacogenetics?" Clin Pharmacol Ther 95(3): 245-247. Delaney, J. A. C., L. Opatrny, et al. (2006). "Warfarin use and the risk of motor vehicle crash in older drivers." British Journal of Clinical Pharmacology 61(2): 229-232.

Ellen, R. L., S. C. Marshall, et al. (2006). "Systematic review of motor vehicle crash risk in persons with sleep apnea." J Clin Sleep Med 2(2): 193-200.

Gibson, J. E., R. B. Hubbard, et al. (2009). "Use of Self-controlled Analytical Techniques to Assess the Association Between Use of Prescription Medications and the Risk of Motor Vehicle Crashes." American Journal of Epidemiology 169(6): 761-768.

Greenland, S. (1996). "Confounding and exposure trends in case-crossover and case-time-control designs." Epidemiology 7(3): 231-239. 
Greenland, S. (1999). "A unified approach to the analysis of case-distribution (case-only) studies." Stat Med 18(1): 1-15.

Gupta, A. and S. Li (2013). "Safety and efficacy of once-daily gastroretentive gabapentin in patients with postherpetic neuralgia aged 75 years and over." Drugs Aging 30(12): 999-1008.

Gustavsen, I., J. G. Bramness, et al. (2008). "Road traffic accident risk related to prescriptions of the hypnotics zopiclone, zolpidem, flunitrazepam and nitrazepam." Sleep Medicine 9(8): 818-822.

Hansen, R. N. (2012). Investigating the Association Between Sedative Hypnotic Prescription Exposures and Motor Vehicle Crashes. 3521668 Ph.D., University of Washington

Hansotia, P. and S. K. Broste (1991). "The effect of epilepsy or diabetes mellitus on the risk of automobile accidents." N Engl J Med 324(1): 22-26.

Hemmelgarn, B., L. E. Lévesque, et al. (2006). "Anti-diabetic drug use and the risk of motor vehicle crash in the elderly." Canadian Journal of Clinical Pharmacology 13(1): e112-e120.

Higgins, J. P., S. W. Wright, et al. (1996). "Alcohol, the elderly, and motor vehicle crashes." Am J Emerg Med 14(3): 265-267.

Innominato, P. F., V. P. Roche, et al. (2014). "The circadian timing system in clinical oncology." Ann Med 46(4): 191-207.

Joy, M. S., R. F. Frye, et al. (2014). "In vivo alterations in drug metabolism and transport pathways in patients with chronic kidney diseases." Pharmacotherapy 34(2): 114-122.

Keall, M. D. and W. J. Frith (2004). "Older driver crash rates in relation to type and quantity of travel." Traffic Inj Prev 5(1): 26-36.

Kelly, E., S. Darke, et al. (2004). "A review of drug use and driving: epidemiology, impairment, risk factors and risk perceptions." Drug Alcohol Rev 23(3): 319-344.

Lonnen, K. F., R. J. Powell, et al. (2008). "Road traffic accidents and diabetes: insulin use does not determine risk." Diabet Med 25(5): 578-584.

Lotfipour, S. and F. Vaca (2007). "Commentary: Polypharmacy and older drivers: beyond the doors of the emergency department (ED) for patient safety." Ann Emerg Med 49(4): 535-537. 
Lumley, T. and D. Levy (2000). "Bias in the case crossover design implications for studies of air pollution." Environmetrics 11: 689-704.

Maclure, M. (1991). "The case-crossover design: a method for studying transient effects on the risk of acute events." Am J Epidemiol 133(2): 144-153.

Markert, C., P. Ngui, et al. (2014). "Influence of St. John's wort on the steady-state pharmacokinetics and metabolism of bosentan." Int J Clin Pharmacol Ther 52(4): 328-336.

Martin, R., K. Meador, et al. (2001). "Comparative cognitive effects of carbamazepine and gabapentin in healthy senior adults." Epilepsia 42(6): 764-771.

Massie, D. L., K. L. Campbell, et al. (1995). "Traffic accident involvement rates by driver age and gender." Accid Anal Prev 27(1): 73-87.

McGwin, G., Jr., R. V. Sims, et al. (2000). "Relations among chronic medical conditions, medications, and automobile crashes in the elderly: a population-based case-control study." Am J Epidemiol 152(5): 424-431.

Mintzer, M. Z., R. K. Lanier, et al. (2010). "Effects of repeated tramadol and morphine administration on psychomotor and cognitive performance in opioid-dependent volunteers." Drug Alcohol Depend 111(3): 265-268.

Mittleman, M. A., M. Maclure, et al. (1995). "Control sampling strategies for case-crossover studies: an assessment of relative efficiency." Am J Epidemiol 142(1): 91-98.

Orriols, L., P. Philip, et al. (2011). Benzodiazepine-like hypnotics and the associated risk of road traffic accidents. Clin Pharmacol Ther. United States. 89: 595-601.

Rothman, K. J., S. Greenland, et al. (2008). Modern epidemiology-third edition,. Philadelphia, Lippincott Williams and Wilkins,.

Rudisill, T. M., S. Zhao, et al. (2014). "Trends in drug use among drivers killed in U.S. traffic crashes, 1999-2010." Accid Anal Prev 70: 178-187.

Sagberg, F. (2006). "Driver health and crash involvement: a case-control study." Accid Anal Prev 38(1): 28-34. 
SAS Institute (2010). Cary, NC.

Siegmund, W., J. Siegert, et al. (2012). "Influence of a Fat-Rich Meal on Bioavailability of ExtendedRelease and Immediate-Release Propiverine." The Journal of Clinical Pharmacology 52(5): 681690.

Sims, R. V., G. McGwin, Jr., et al. (2000). "Exploratory study of incident vehicle crashes among older drivers." Journals of Gerontology: Series A: Biological Sciences and Medical Sciences $\mathbf{5 5 A}(1)$ : M22-M27.

Skurtveit, S., H. Strøm, et al. (2009). "Road traffic accident risk in patients with diabetes mellitus receiving blood glucose-lowering drugs. Prospective follow-up study." Diabetic Medicine 26(4): 404-408.

Stein, C. and C. Baerwald (2014). "Opioids for the treatment of arthritis pain." Expert Opin Pharmacother 15(2): 193-202.

Uc, E. Y., M. Rizzo, et al. (2006). "Driving with distraction in Parkinson disease." Neurology 67(10): 1774-1780.

Walsh, J. M., J. J. de Gier, et al. (2004). "Drugs and driving." Traffic Inj Prev 5(3): 241-253.

Wang, P. S., S. Schneeweiss, et al. (2004). "Use of the case-crossover design to study prolonged drug exposures and insidious outcomes." Ann Epidemiol 14(4): 296-303.

West Virginia Department of Health and Human Resources. (2010). "West Virginia Vital Statistics 2010." Retrieved 11/20/2013, from www.wvdhhr.org/bph/hsc/pubs/vital/2010/2010Vital.pd.

West Virginia Department of Health Bureau of Family Planning (2010). West Virginia Vital Statistics 2010. Charleston WV, Department of Health and Human Services,.

West Virginia University Healthcare (2013). Community health needs assessment and report. Morgantown WV,.

Wilson, F. A., J. P. Stimpson, et al. (2014). "Fatal crashes from drivers testing positive for drugs in the U.S., 1993-2010." Public Health Rep 129(4): 342-350. 
Yang, Y. H., J. N. Lai, et al. (2011). "Increased risk of hospitalization related to motor vehicle accidents among people taking zolpidem: a case-crossover study." J Epidemiol 21(1): 37-43.

Zhu, M., S. Zhao, et al. (2013). "Appalachian versus non-Appalachian U.S. traffic fatalities, 2008-2010." Ann Epidemiol 23(6): 377-380.

\section{Chapter 5}

Baker, D. W., J. A. Gazmararian, et al. (2000). "The Association Between Age and Health Literacy Among Elderly Persons." The Journals of Gerontology Series B: Psychological Sciences and Social Sciences 55(6): S368-S374.

Blanchard, R. A., A. M. Myers, et al. (2010). "Correspondence between self-reported and objective measures of driving exposure and patterns in older drivers." Accid Anal Prev 42(2): 523-529.

D’Ambrosio, L. A., L. K. Donorfio, et al. (2008). "Gender Differences in Self-Regulation Patterns and Attitudes Toward Driving Among Older Adults." Journal of Women \& Aging 20(3-4): 265-282.

Fulton, M. M. and E. R. Allen (2005). "Polypharmacy in the elderly: a literature review." J Am Acad Nurse Pract 17(4): 123-132.

Kelly, R., T. Warke, et al. (1999). "Medical restrictions to driving: the awareness of patients and doctors." Postgrad Med J 75(887): 537-539. 


\section{Figures}

\section{Chapter 2}

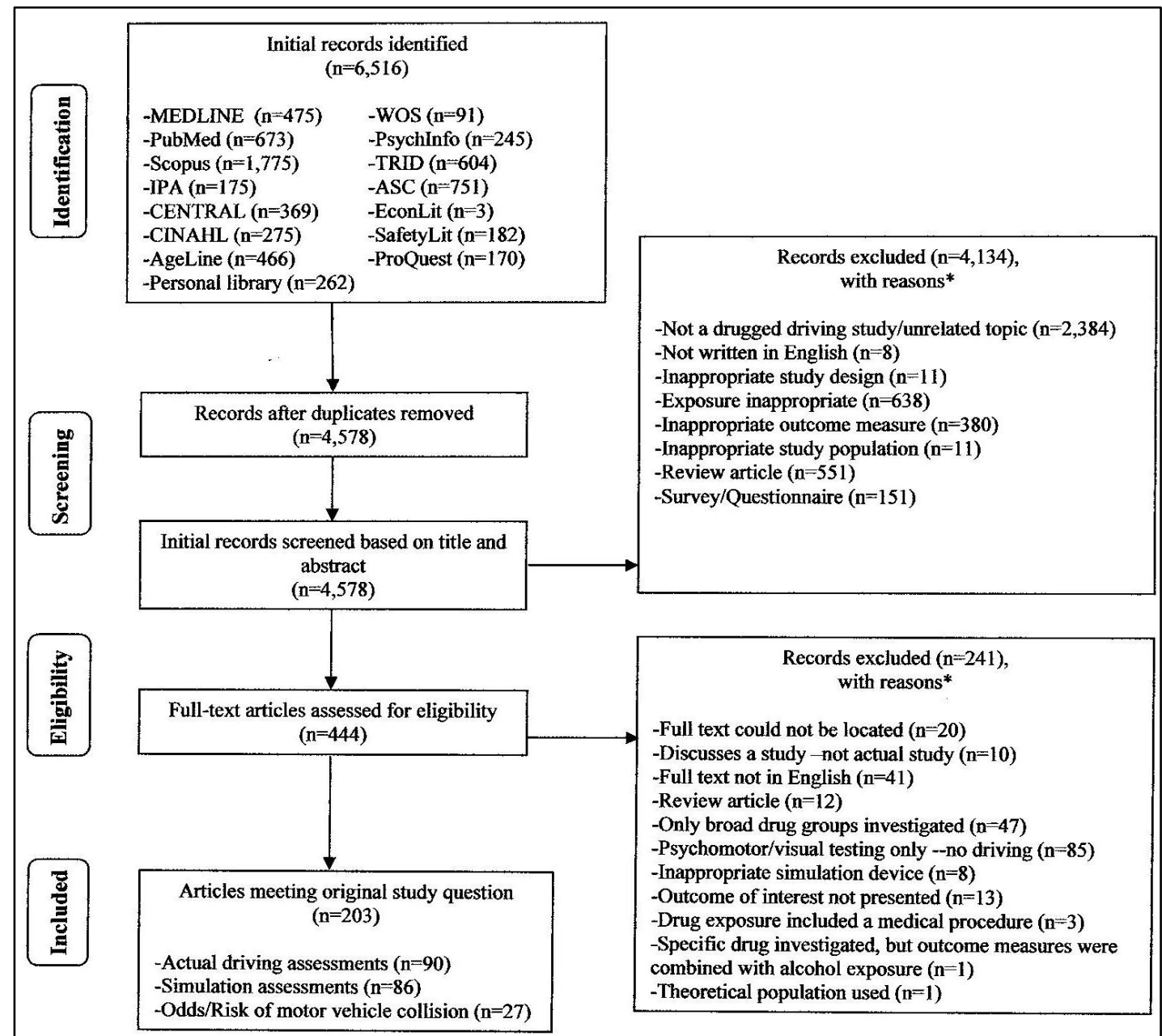

Figure 1 Flow chart for the selection of studies. *, studies could have been excluded for more than one reason 


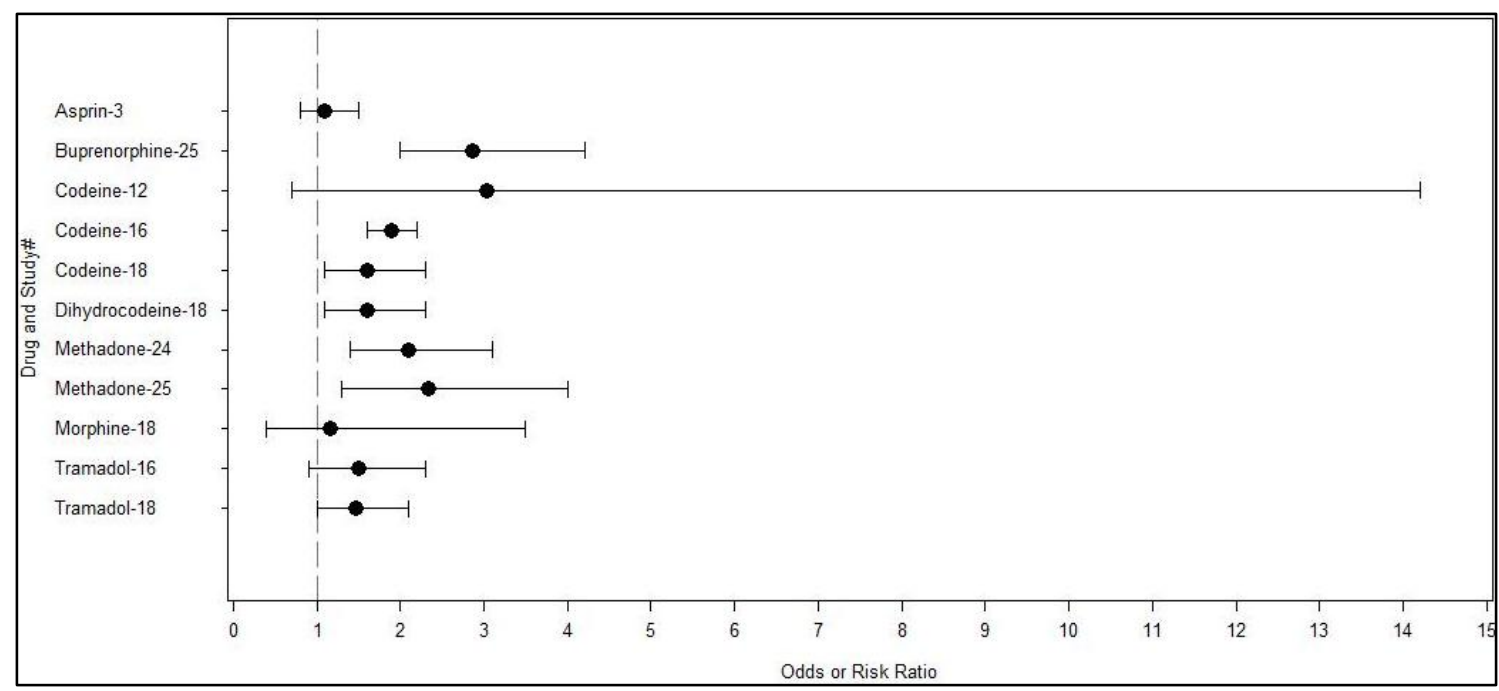

Figure 2. Odds or risk ratios with corresponding 95\% confidence intervals from included studies that investigated the association between specific analgesic medications and MVC. Estimates by Foley et al (Aspirin-3) were adjusted for age and gender; all other estimates presented are unadjusted.

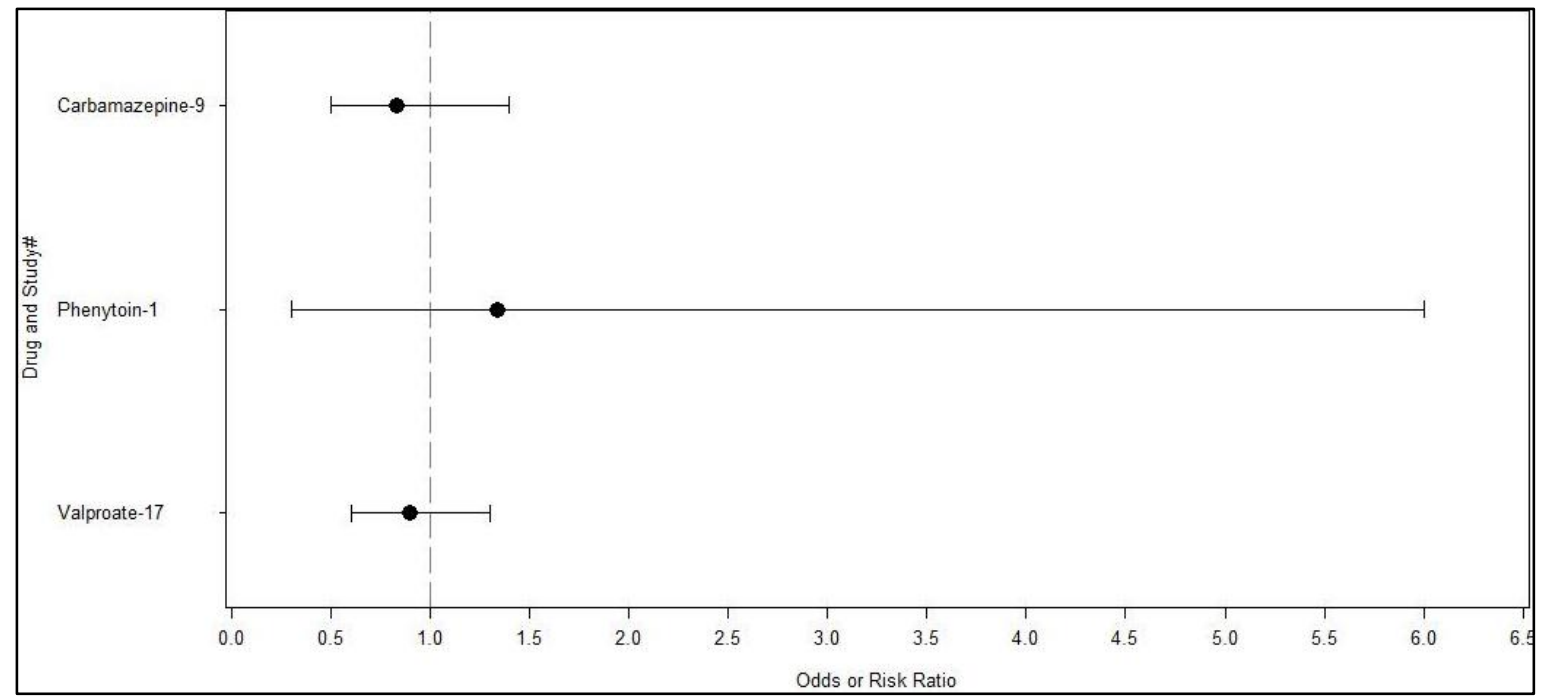

Figure 3. Odds or risk ratios with corresponding 95\% confidence intervals from included studies that investigated the association between specific anticonvulsant medications and MVC. The study by Etminan et al (Carbazepine-9) was adjusted for age, sex, residence, previous MVC, chronic disease score, and exposure to antidepressants, antiepileptic, benzodiazepines, antipsychotics, antimigraine, muscle relaxants, and/or narcotic analgesics. All other estimates are unadjusted. 


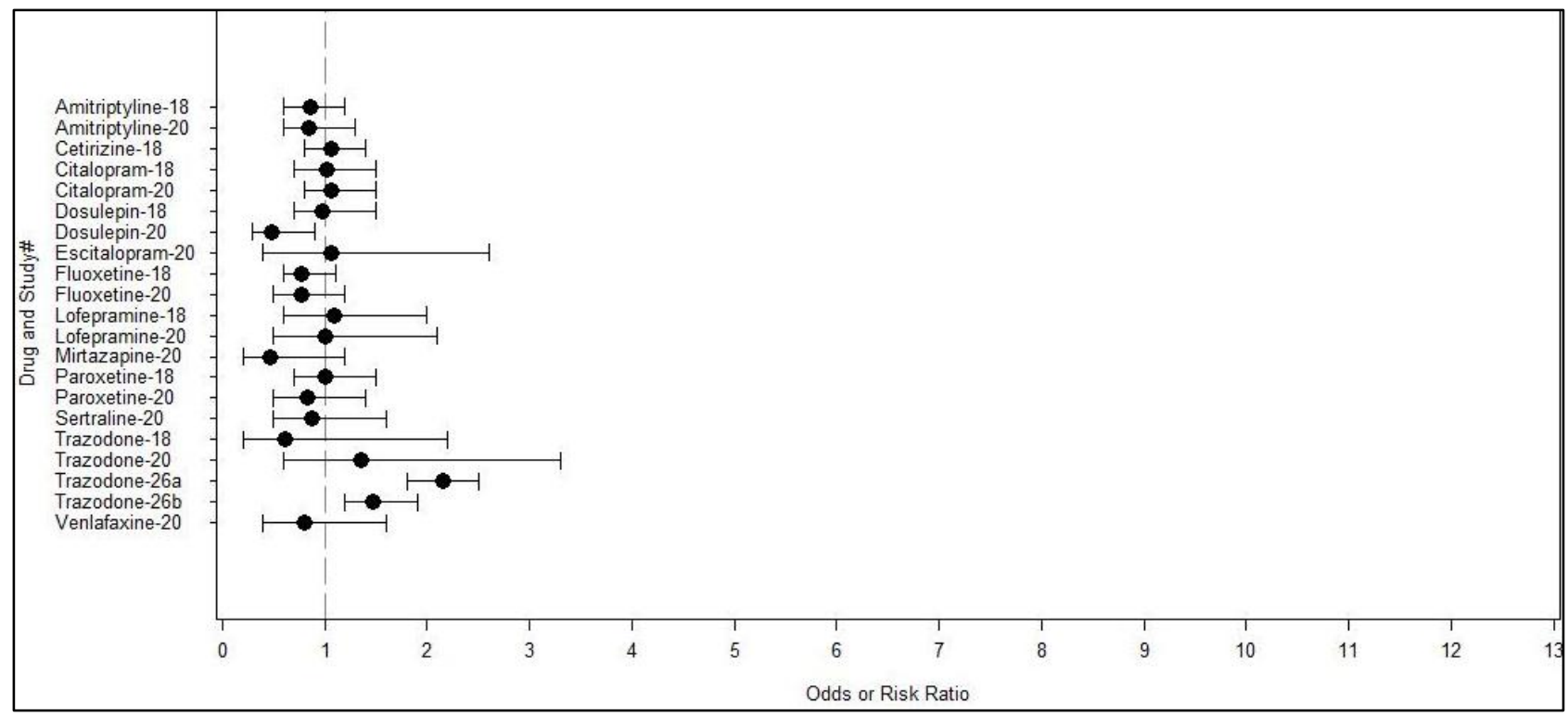

Figure 4. Odds or risk ratios with corresponding 95\% confidence intervals from included studies that investigated the association between specific antidepressant medications and MVC. The study by Hansen (Trazadone-26) provided two estimates. The first estimate (26a) corresponds to new users of Trazodone while the second (26b) corresponds to prevalent users of Trazodone.

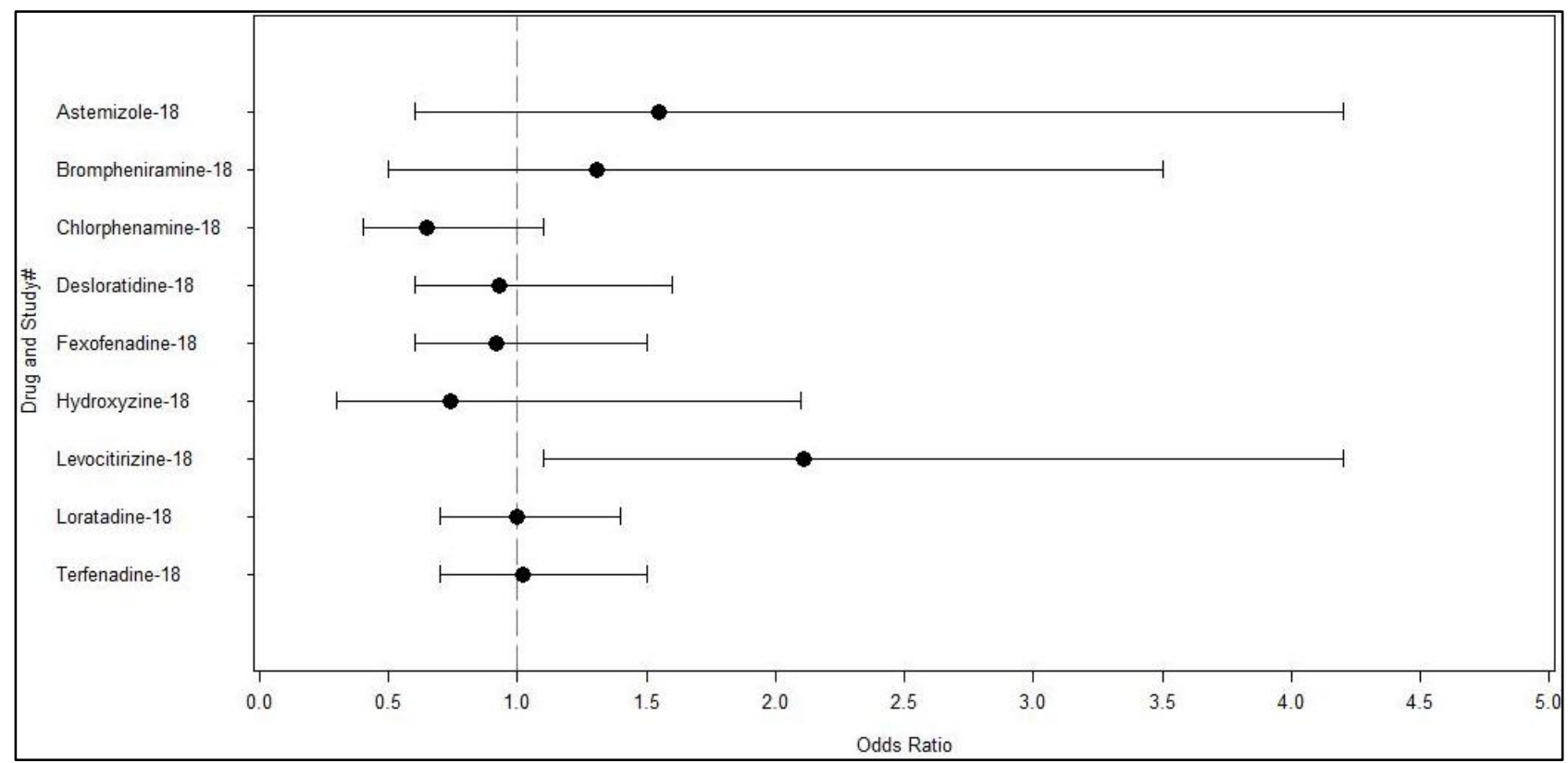

Figure 5. Odds or risk ratios with corresponding 95\% confidence intervals from included studies that investigated the association between specific antihistamine medications and MVC. All estimates are unadjusted. 


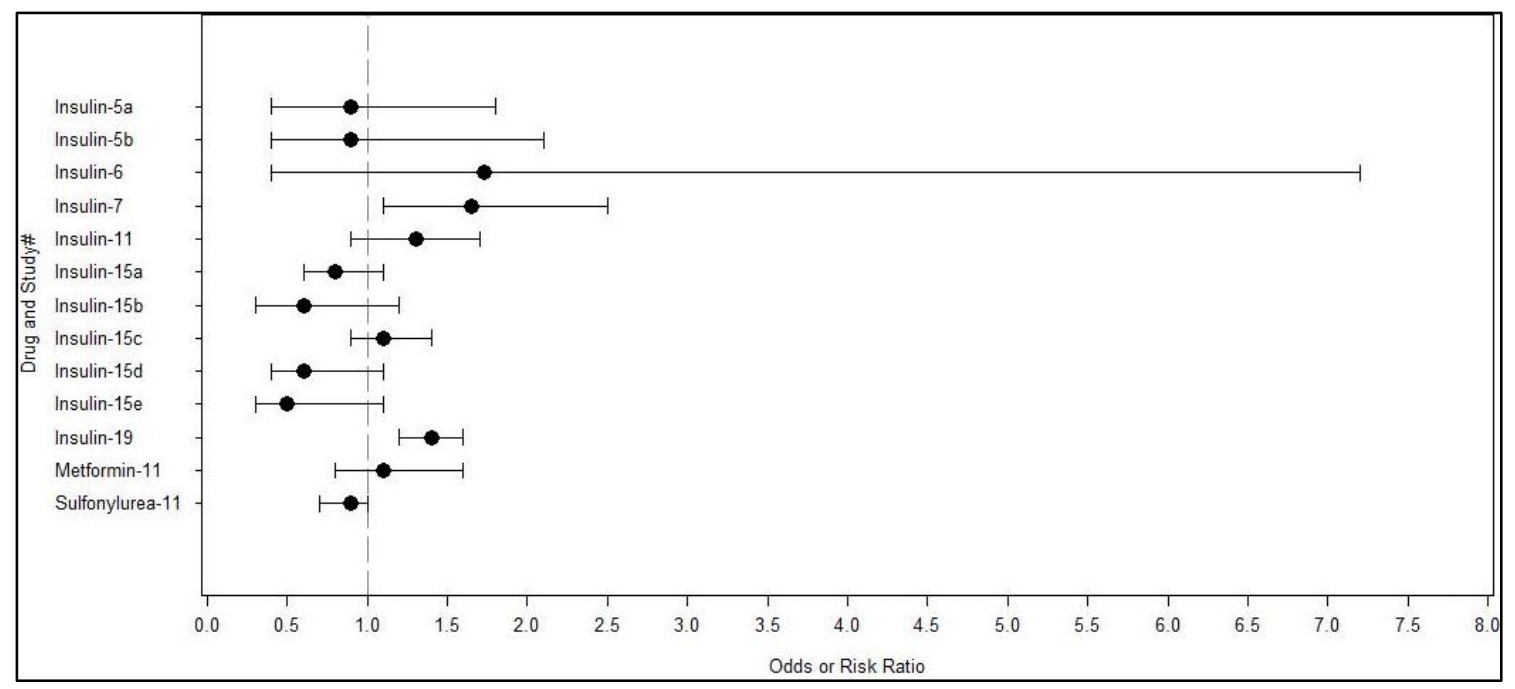

Figure 6. Odds or risk ratios with corresponding 95\% confidence intervals from included studies that investigated the association between specific antihyperglycemic medications and MVC. The study by Lonnen et al provided five estimates of risk (Insulin15a-e). These correspond to the risk of MVC for the following age groups respectively, $<25,25-44,45-64,65-74$, and 75-84 years of age. The study by Mcgwin et al provided two estimates of risk (Insulin-5a-b), which corresponds with the odds of having any crash or a not-at-fault crash, respectively. The study by Mcgwin et al was adjusted for age, sex, and annual miles driven. The study by Sims et al was adjusted for sex, age, and days driven per week (Insulin-6). The study by Hemmelgarn et al was adjusted for age, gender, previous MVC, and place of residence (Insulin-11). All other estimates are unadjusted.

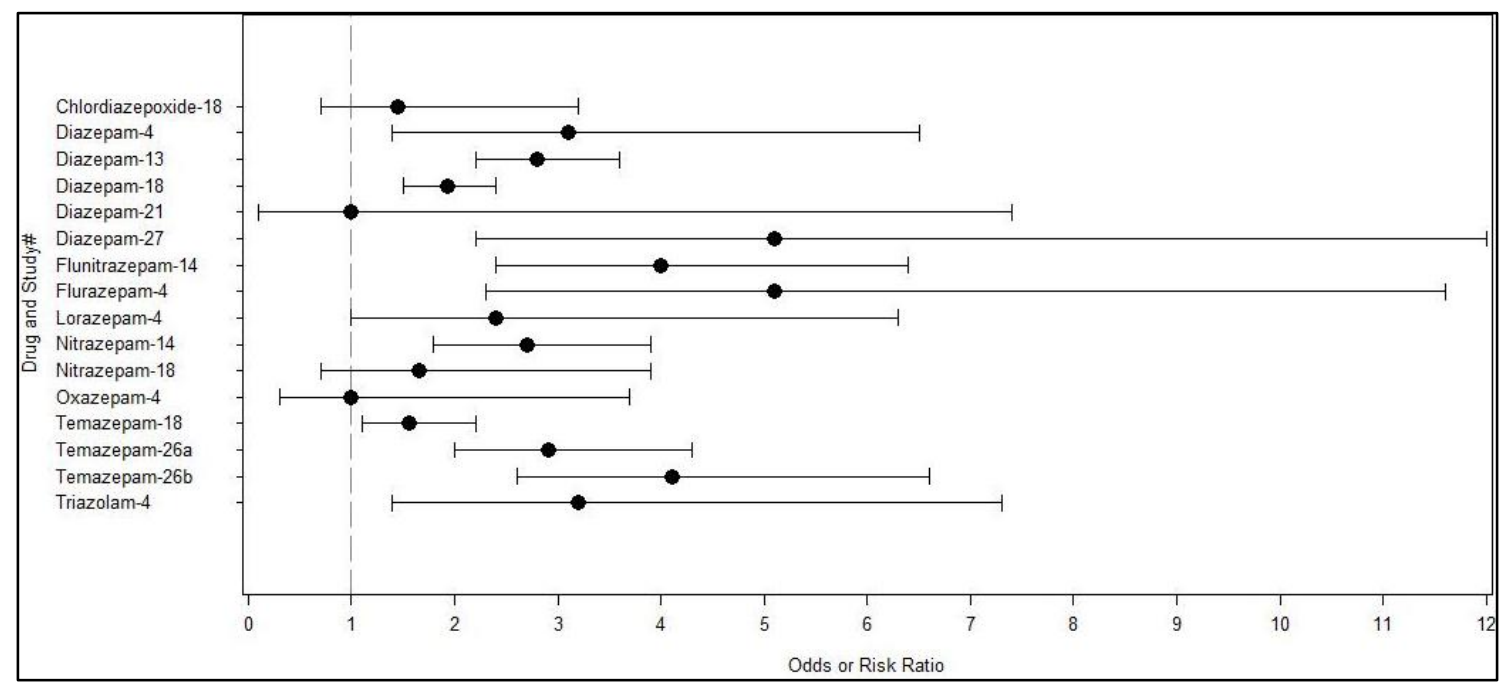

Figure 7. Odds or risk ratios with corresponding 95\% confidence intervals from included studies that investigated the association between specific benzodiazepine medications and MVC. Hansen provided two estimates of risk (Temazepam-26a-b) which correspond to new and prevalent users of Temazepam, respectively. All other estimates are unadjusted. 


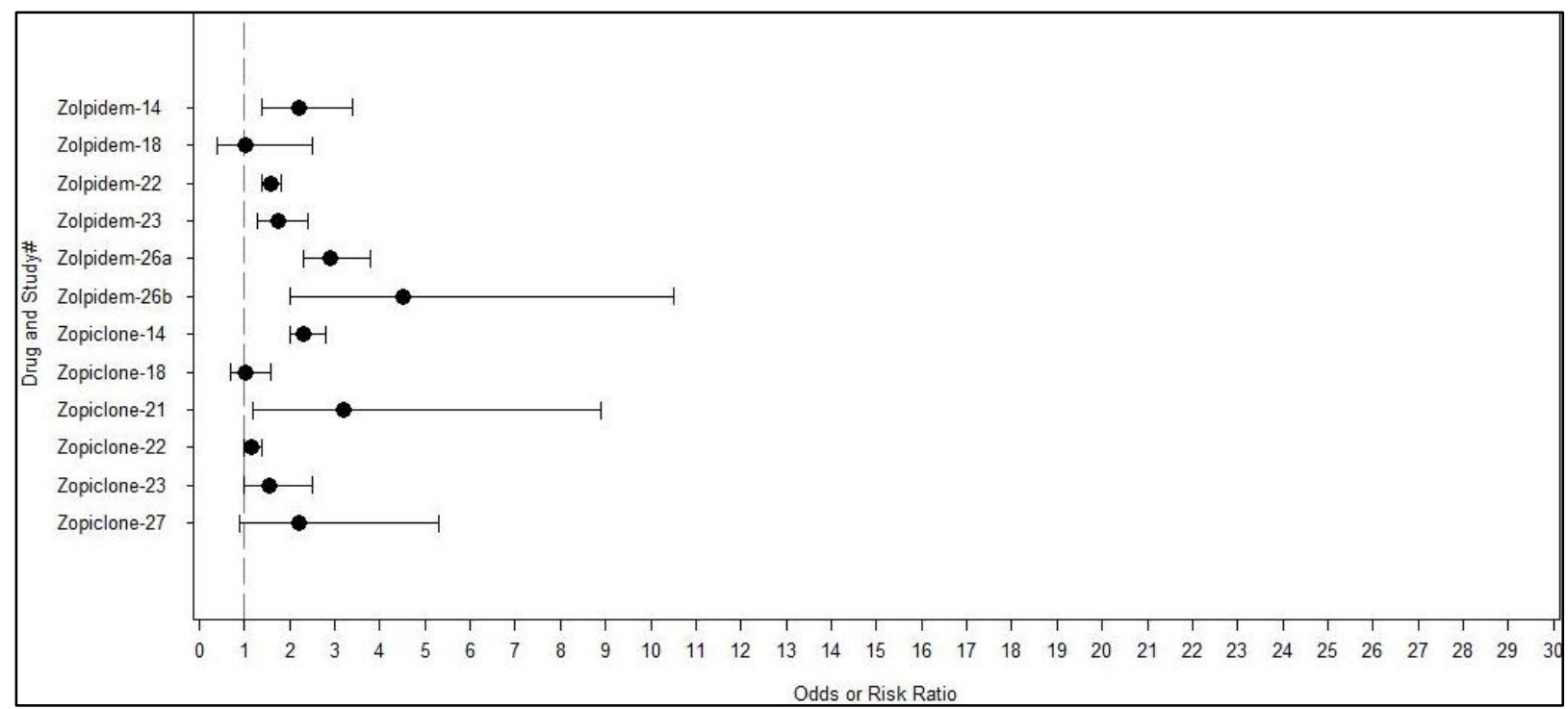

Figure 8. Odds or risk ratios with corresponding 95\% confidence intervals from included studies that investigated the association between specific sleep-promoting medications and MVC. Hansen (Zolpidem-26a-b) provided two estimates of risk which correspond to new and prevalent users of Zolpidem, respectively. The estimates provided by Yang et al (Zolpidem-23 and Zopiclone-23) were adjusted for concomitant use of the following medications: Zolpidem, Zopiclone, long and short acting benzodiazepines, antihistamines, anticonvulsants, antidepressants, other sedatives/hypnotics, other psychoactive drugs, muscle relaxants, and opioid analgesics. All other estimates are unadjusted.

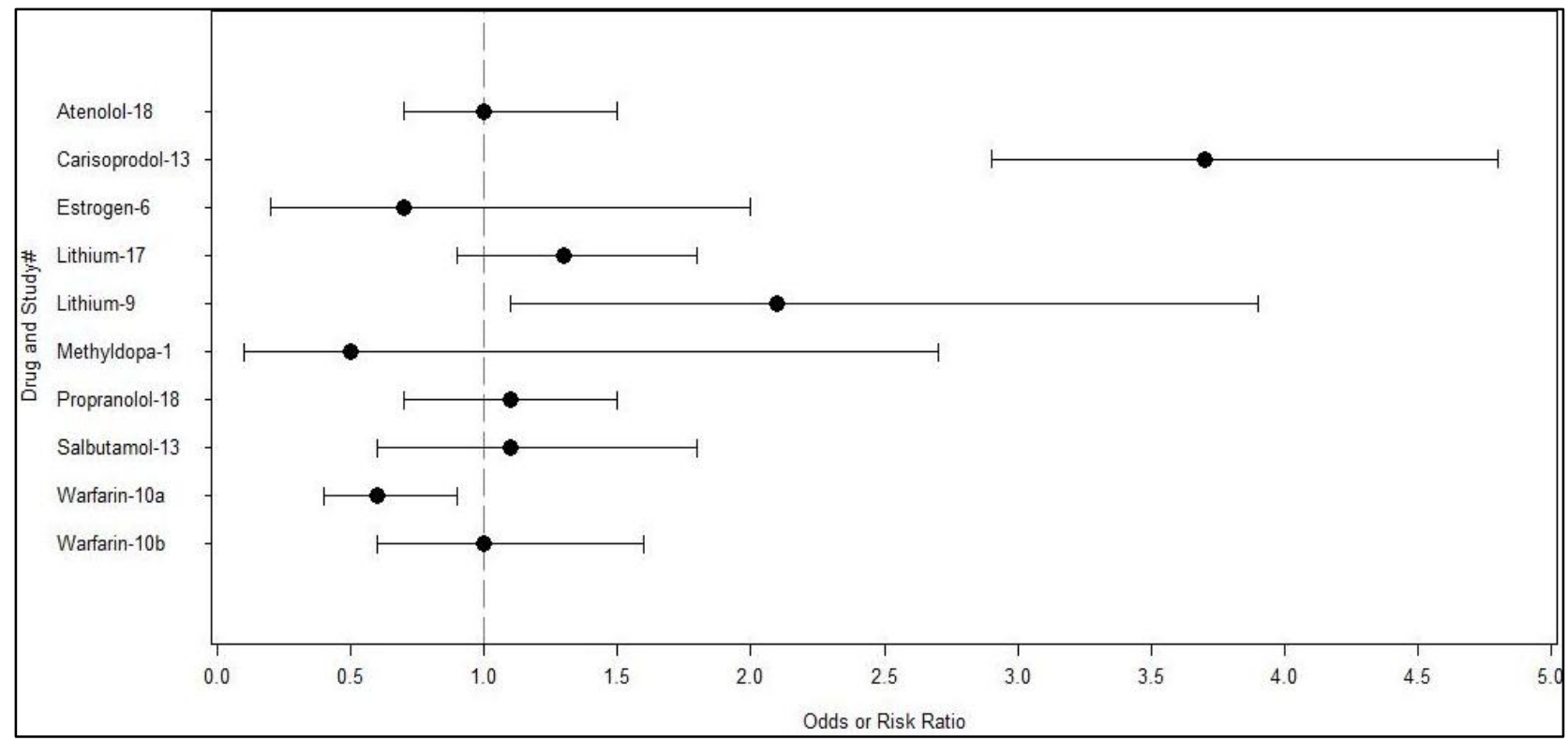

Figure 9. Odds or risk ratios with corresponding 95\% confidence intervals from included studies that investigated the association between MVC and specific medications that could not be grouped into the other drug categories. Delaney et al provided two estimates of risk (Warfarin-10a-b) which correspond to both new and prevalent users, respectively, of Warfarin. The study by Delaney et al was adjusted for cardiac or stroke events within the past year and the following drug classes in the previous 60 days: antidepressants, anti-epileptics, benzodiazepines, antipsychotics, anti-migraine, narcotic analgesics, muscle relaxants. The study by Sims et al was adjusted for sex, age, and days driven per week (Estrogen-6). The study by Etminan et al was adjusted for age, sex, residence, previous MVC, chronic disease score, and exposure to antidepressants, antiepileptic, benzodiazepines, antipsychotics, antimigraine, muscle relaxants, and/or narcotic analgesics (Lithium-9). All other estimates are unadjusted. 


\section{Chapter 3}

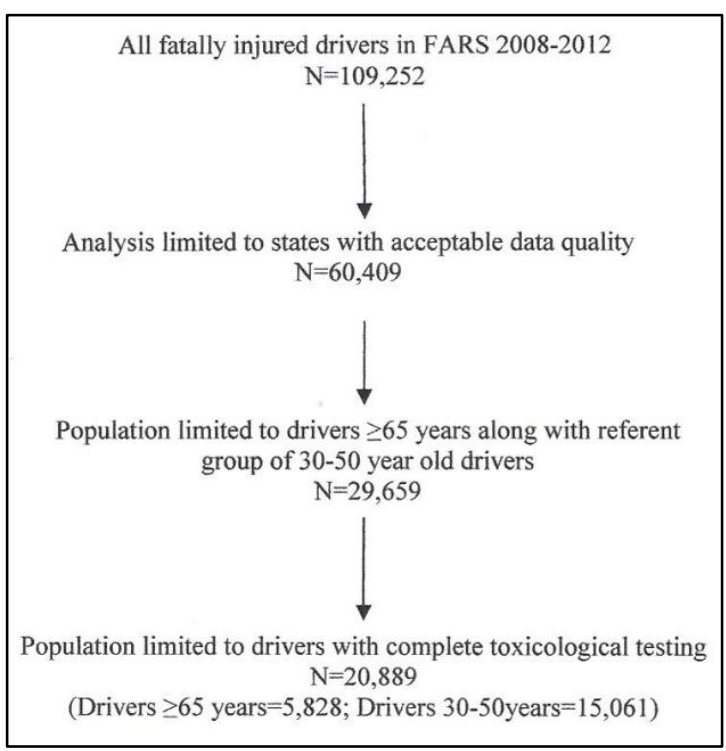

Figure 1. Overview of participant selection with sample sizes

\section{Chapter 4}

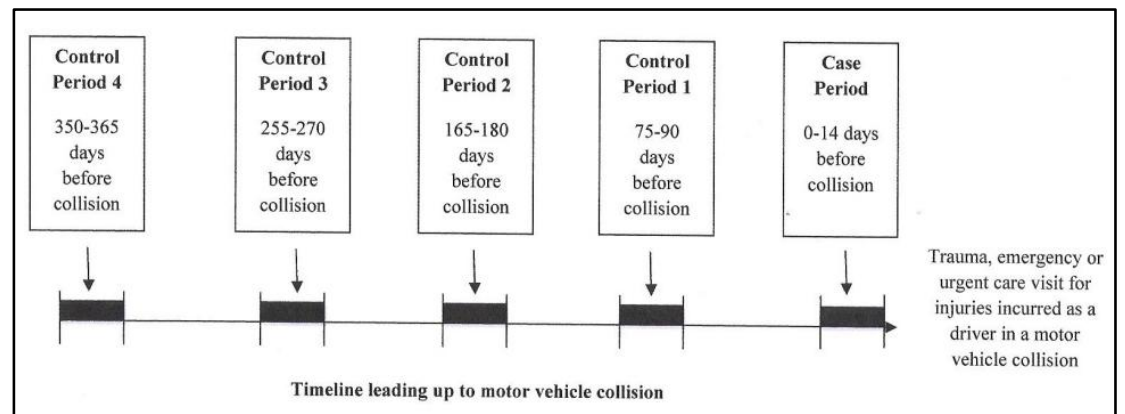

Figure 1. Overview of sampling schema for case and control periods 


\section{Tables}

\section{Chapter 2}

\begin{tabular}{|c|c|c|c|c|c|c|c|c|}
\hline $\begin{array}{c}\text { Study } \\
\#\end{array}$ & Author & Year & $\begin{array}{c}\text { Country of } \\
\text { Origin }\end{array}$ & Study Design & $\begin{array}{l}\text { Outcome } \\
\text { Measure }\end{array}$ & $\begin{array}{c}\text { Population* } \\
\text { Characteristics }\end{array}$ & Medications Investigated & $\begin{array}{c}\text { Quality } \\
\text { Score }\end{array}$ \\
\hline 1 & Nelson & 1992 & U.S. & Case-Control & Odds Ratio & $30-65 \mathrm{yrs}$, females & Methyldopa, Phenytoin & $5 / 5$ \\
\hline 2 & Koepsell et al & 1994 & U.S. & Case-Control & Odds Ratio & $\geq 65$ yrs, both genders & Insulin & $5 / 5$ \\
\hline 3 & Foley et al & 1995 & U.S. & Cohort & \begin{tabular}{|l} 
Relative Risk \\
\end{tabular} & $\geq 65 \mathrm{yrs}$, both genders & Aspirin & $5 / 5$ \\
\hline 4 & Neutel & 1998 & Canada & Case-Control & Odds Ratio & $\geq 20 \mathrm{yrs}$, both genders & $\begin{array}{l}\text { Diazepam, Flurazepam, Lorazepam, Oxazepam, } \\
\text { Triazolam }\end{array}$ & $4 / 5$ \\
\hline 5 & McGwin et al & 2000 & U.S. & Case-Control & Odds Ratio & $\geq 65$ yrs, both genders & Insulin & $5 / 5$ \\
\hline 6 & Sims et al & 2000 & U.S. & Cohort & Relative Risk & $\geq 55$ yrs, both genders & Estrogen, Insulin & $5 / 5$ \\
\hline 7 & Cui & 2001 & Canada & Case-Control & Odds Ratio & $\geq 65.5 \mathrm{yrs}$, both genders & Insulin & $5 / 5$ \\
\hline 8 & Mura et al & 2003 & France & Case-Control & Odds Ratio & $\geq 18$ yrs, both genders & Morphine & $4 / 5$ \\
\hline 9 & Etminan et al & 2004 & Canada & Case-Control & Odds Ratio & $67-84$ yrs, both genders & Carbamazepine, Lithium & $5 / 5$ \\
\hline 10 & Delaney et al & 2006 & Canada & Case-Control & Odds Ratio & $67-84$ yrs, both genders & Warfarin & $5 / 5$ \\
\hline 11 & Hemmelgarn et al & 2006 & Canada & Case-Control & Odds Ratio & $67-84 \mathrm{yrs}$, both genders & Insulin, Metformin, Sulfonylurea & $5 / 5$ \\
\hline 12 & Mathijssen et al & 2006 & Netherlands & Case-Control & Odds Ratio & $\geq 18 \mathrm{yrs}$, both genders & Codeine & $4 / 5$ \\
\hline 13 & Bramness et al & 2007 & Norway & Cohort & Relative Risk & $18-69 \mathrm{yrs}$, both genders & Carisoprodol, Diazepam, Salbutamol & $5 / 5$ \\
\hline 14 & Gustavsen et al & 2008 & Norway & Cohort & Relative Risk & $18-69 \mathrm{yrs}$, both genders & Flunitrazepam, Nitrazepam, Zolpidem, Zopiclone & $5 / 5$ \\
\hline 15 & Lonnen et al & 2008 & England & Cohort & Relative Risk & $\geq 15$ yrs, both genders & 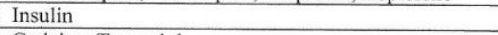 & $4 / 5$ \\
\hline 16 & Bachs et al & 2009 & Norway & Cohort & Relative Risk & $18-70$ yrs, both genders & Codeine, Tramadol & $5 / 5$ \\
\hline 17 & Bramness et al & 2009 & Norway & Cohort & Relative Risk & $18-70$ yrs, both genders & Lithium, Valproate & $5 / 5$ \\
\hline 18 & Gibson et al & 2009 & England & Case-Crossover & Odds Ratio & 18-74 yrs, both genders & $\begin{array}{l}\text { Amitryptyline, Astemizole, Atenolol, } \\
\text { Brompheniramine, Cetirizine, Chlordiazepoxide } \\
\text { Chlorphenamine Citalopram Codeine, Desloratidine, } \\
\text { Diazepam, Dihydrocodeine, Doselupin, Fexofenadine, } \\
\text { Fluoxetine, Hydroxyzine, Levocitirizine,, } \\
\text { Lofepramine, Loratadine, Morphine, Nitrazepam, } \\
\text { Paroxetine, Propranolol, Temazepam, Terfendine, } \\
\text { Tramadol, Trazodone, Zopiclone, Zolpidem }\end{array}$ & $5 / 5$ \\
\hline 19 & Skurtveit et al & 2009 & Norway & Cohort & Relative Risk & $18-69$ yrs, both genders & Insulin & $5 / 5$ \\
\hline 20 & Coupland et al & 2011 & England & Cohort & Relative Risk & $\geq 65 \mathrm{yrs}$, both genders & $\begin{array}{l}\text { Amitiptyline, Citalopram, Dosulepin, Escitalopram, } \\
\text { Fluoxetine, Lofepramine, Mirtazapine, Paroxetine, } \\
\text { Sertraline, Trazodone, Venlafaxine }\end{array}$ & $5 / 5$ \\
\hline 21 & Gjerde et al & 2011 & Norway & Case-Control & Odds Ratio & Both genders & Diazepam, Zopiclone & $5 / 5$ \\
\hline 22 & Orriols et al & 2011 & France & Case-Control & Odds Ratio & Both genders & Zopiclone, Zolpidem & $5 / 5$ \\
\hline 23 & Yang et al & 2011 & Taiwan & Case-Crossover & Odds Ratio & $\geq 18$ yrs, both genders & Zopiclone, Zolpidem & $5 / 5$ \\
\hline 24 & Bramness et al & 2012 & Norway & Cohort & Relative Risk & $18-69$ yrs, both genders & Methadone & $5 / 5$ \\
\hline 25 & Corsenac et al & 2012 & France & Case-Control & Odds Ratio & Both genders & Buprenorphine, Methadone & $5 / 5$ \\
\hline 26 & Hansen & 2012 & U.S. & Cohort & Relative Risk & 21-79 yrs, both genders & Temazepam, Trazodone, Zolpidem & $5 / 5$ \\
\hline 27 & Gjerde et al & 2013 & Norway & Case-Control & Odds Ratio & Both genders & Diazepam, Zopiclone & $5 / 5$ \\
\hline
\end{tabular}




\section{Chapter 3}

Table 1. Characteristics of fatally injured drivers $\geq 65$ years with known drug test results ${ }^{\mathrm{a}}$

\begin{tabular}{|c|c|c|c|c|c|c|}
\hline \multirow[t]{2}{*}{ Characteristic } & \multicolumn{2}{|c|}{$\begin{array}{c}\text { Drivers } \\
\geq 65 \text { years who tested } \\
\text { negative for drugs } \\
(\mathrm{N}=4,789)\end{array}$} & \multicolumn{2}{|c|}{$\begin{array}{c}\text { Drivers } \\
\geq 65 \text { years who tested } \\
\text { positive for drugs } \\
(\mathrm{N}=1,039)\end{array}$} & \multicolumn{2}{|c|}{$\begin{array}{c}\text { Total Drivers } \\
\geq 65 \text { years with } \\
\text { known test results } \\
(\mathrm{N}=5,828)\end{array}$} \\
\hline & $\begin{array}{l}\text { No. of } \\
\text { drivers }\end{array}$ & $\%$ & $\begin{array}{l}\text { No. of } \\
\text { drivers }\end{array}$ & $\%$ & $\begin{array}{l}\text { No. of } \\
\text { drivers }\end{array}$ & $\%$ \\
\hline \multicolumn{7}{|l|}{ Age (in years) } \\
\hline $65-69$ & 1,306 & 27.3 & 353 & 34.0 & 1,659 & 28.5 \\
\hline $70-79$ & 1,885 & 39.4 & 431 & 41.5 & 2,316 & 39.7 \\
\hline $80-89$ & 1,382 & 28.9 & 229 & 22.0 & 1,611 & 27.6 \\
\hline$\geq 90$ & 216 & 4.5 & 26 & 2.5 & 242 & 4.2 \\
\hline \multicolumn{7}{|l|}{ Gender } \\
\hline Male & 3,309 & 69.1 & 737 & 70.9 & 4,046 & 69.4 \\
\hline Female & 1,480 & 30.9 & 302 & 29.1 & 1,782 & 30.6 \\
\hline \multicolumn{7}{|l|}{ Race } \\
\hline White & 3,466 & 91.3 & 804 & 92.3 & 4,270 & 91.5 \\
\hline African American & 239 & 6.3 & 49 & 5.6 & 288 & 6.2 \\
\hline Asian & 57 & 1.5 & 9 & 1.0 & 66 & 1.4 \\
\hline Native American & 30 & 0.8 & 6 & 0.7 & 36 & 0.8 \\
\hline Other & 3 & 0.1 & 3 & 0.3 & 6 & 0.1 \\
\hline Unknown & 994 & & 168 & & 1162 & \\
\hline \multicolumn{7}{|l|}{ Ethnicity } \\
\hline Hispanic & 132 & 3.5 & 21 & 2.4 & 153 & 3.3 \\
\hline Non-Hispanic & 3,642 & 96.5 & 850 & 97.6 & 4,492 & 96.7 \\
\hline Unknown & 1,015 & & 168 & & 1,183 & \\
\hline \multicolumn{7}{|l|}{ Type of drug test given } \\
\hline Urine & 172 & 3.7 & 40 & 3.9 & 212 & 3.7 \\
\hline Blood & 4,336 & 92.5 & 864 & 85.2 & 5,200 & 91.2 \\
\hline Urine \& Blood & 180 & 3.8 & 110 & 10.9 & 290 & 5.1 \\
\hline Unknown & 101 & & 25 & & 126 & \\
\hline \multicolumn{7}{|l|}{$\begin{array}{l}\text { Blood alcohol concentration } \\
(\mathrm{g} / \mathrm{dl})\end{array}$} \\
\hline 0 & 4,327 & 90.0 & 915 & 88.0 & 5,242 & 90.0 \\
\hline $0.01-0.07$ & 149 & 3.0 & 46 & 4.0 & 195 & 3.0 \\
\hline$\geq 0.08$ & 313 & 7.0 & 78 & 7.0 & 391 & 7.0 \\
\hline \multicolumn{7}{|l|}{$\begin{array}{l}\text { Number of drugs detected at } \\
\text { time of } \operatorname{crash}^{b}\end{array}$} \\
\hline 0 & 4,789 & 100.0 & NA & & 4,789 & 82.2 \\
\hline 1 & NA & & 653 & 62.9 & 653 & 11.2 \\
\hline 2 & NA & & 241 & 23.2 & 241 & 4.1 \\
\hline 3 & NA & & 145 & 14.0 & 145 & 2.5 \\
\hline \multicolumn{7}{|l|}{$\begin{array}{l}\text { DWI conviction within past } \\
3 \text { years }^{c}\end{array}$} \\
\hline No & 4,717 & 99.5 & 1,023 & 99.2 & 5,740 & 99.5 \\
\hline Yes & 23 & 0.5 & 8 & 0.8 & 31 & 0.5 \\
\hline Unknown & 49 & & 8 & & 57 & \\
\hline \multicolumn{7}{|l|}{ Crash within past 3 years } \\
\hline No & 4,127 & 89.4 & 908 & 88.8 & 5,035 & 89.3 \\
\hline Yes & 489 & 10.6 & 115 & 11.2 & 604 & 10.7 \\
\hline
\end{tabular}




\begin{tabular}{|c|c|c|c|c|c|c|}
\hline Unknown & 173 & & 16 & & 189 & \\
\hline \multicolumn{7}{|l|}{ Day of crash } \\
\hline Friday-Sunday & 1,906 & 39.8 & 438 & 42.2 & 2,344 & 40.2 \\
\hline Monday-Thursday & 2,883 & 60.2 & 601 & 57.8 & 3,484 & 59.8 \\
\hline \multicolumn{7}{|l|}{ Time of crash } \\
\hline Day (7:00am-6:59pm) & 3,928 & 82.4 & 831 & 80.4 & 4,759 & 82.0 \\
\hline Night (7:00pm-6:59am) & 839 & 17.6 & 203 & 19.6 & 1,042 & 18.0 \\
\hline Unknown & 22 & & 5 & & 27 & \\
\hline \multicolumn{7}{|l|}{$\begin{array}{l}\text { Number of vehicles } \\
\text { involved }\end{array}$} \\
\hline 1 & 1,762 & 36.8 & 428 & 41.2 & 2,190 & 37.6 \\
\hline$\geq 2$ & 3,027 & 63.2 & 611 & 58.8 & 3,638 & 62.4 \\
\hline \multicolumn{7}{|l|}{$\begin{array}{l}\text { Weather conditions at time } \\
\text { of } \operatorname{crash}^{\mathrm{d}}\end{array}$} \\
\hline Clear & 4,267 & 89.1 & 937 & 90.2 & 5,204 & 89.3 \\
\hline Adverse & 522 & 10.9 & 102 & 9.8 & 624 & 10.7 \\
\hline \multicolumn{7}{|l|}{ Survival time after crash } \\
\hline Death within 1 hour & 2,612 & 57.1 & 553 & 55.0 & 3,165 & 56.7 \\
\hline Death beyond 1 hour & 1,963 & 42.9 & 452 & 45.0 & 2,415 & 43.3 \\
\hline Unknown & 214 & & 34 & & 248 & \\
\hline \multicolumn{7}{|l|}{ Year of crash } \\
\hline 2008 & 1,056 & 22.1 & 166 & 16.0 & 1,222 & 21.0 \\
\hline 2009 & 956 & 20.0 & 191 & 18.4 & 1,147 & 19.7 \\
\hline 2010 & 960 & 20.1 & 194 & 18.7 & 1,154 & 19.8 \\
\hline 2011 & 924 & 19.3 & 243 & 23.4 & 1,167 & 20.0 \\
\hline 2012 & 893 & 18.7 & 245 & 23.6 & 1,138 & 19.5 \\
\hline
\end{tabular}

a:States included in this analysis are AK, AZ, CA, CO, FL, HI, IL, IN, KY, MD, MA, MI, MN, NV, NH ,NJ, NM, NY, ND, OH, PA, RI, SC, VT, VA, WA, WV, WY, and Washington, DC.

b: N/A=not applicable

c: DWI=driving while intoxicated 
Table 2. Prevalence and prevalence ratios for drug presence among sub-groups of fatally injured drivers $\geq 65$ years ${ }^{\mathrm{a}}$

\begin{tabular}{|c|c|c|c|}
\hline Characteristic & $\begin{array}{r}\text { No. of } \\
\text { Drivers } \\
\text { Found } \\
\text { Positive } \\
(\mathrm{N}=1,039) \\
\end{array}$ & $\begin{array}{r}\text { Prevalence Rate } \\
\text { Per } 1,000\end{array}$ & $\begin{array}{r}\text { Prevalence } \\
\text { Ratio } \\
(95 \% \mathrm{CI})^{\mathrm{b}}\end{array}$ \\
\hline \multicolumn{4}{|l|}{ Age (in years) } \\
\hline $65-69$ & 353 & 212.8 & 1.00 (Reference) \\
\hline $70-79$ & 431 & 186.1 & $0.87(0.76,1.01)$ \\
\hline $80-89$ & 229 & 142.5 & $0.67(0.57,0.79)$ \\
\hline$\geq 90$ & 26 & 107.4 & $0.50(0.34,0.75)$ \\
\hline \multicolumn{4}{|l|}{ Gender } \\
\hline Male & 737 & 182.2 & 1.00 (Reference) \\
\hline Female & 302 & 169.5 & $0.93(0.81,1.06)$ \\
\hline \multicolumn{4}{|l|}{ Race } \\
\hline White & 804 & 188.3 & 1.00 (Reference) \\
\hline African American & 49 & 170.1 & $0.90(0.68,1.21)$ \\
\hline Asian & 9 & 136.4 & $0.72(0.38,1.40)$ \\
\hline Native American & 6 & 166.7 & $0.89(0.40,1.98)$ \\
\hline Other & 3 & 500.0 & $2.66(0.85,8.25)$ \\
\hline Unknown & 1,162 & & \\
\hline \multicolumn{4}{|l|}{ Ethnicity } \\
\hline Non-Hispanic & 850 & 189.2 & 1.00 (Reference) \\
\hline Hispanic & 21 & 137.3 & $0.73(0.47,1.12)$ \\
\hline Unknown & 1,183 & & \\
\hline \multicolumn{4}{|l|}{ Type of drug test given } \\
\hline Blood & 864 & 166.2 & 1.00 (Reference) \\
\hline Urine & 40 & 188.7 & $1.14(0.83,1.56)$ \\
\hline Urine \& Blood & 110 & 379.3 & $2.28(1.87,2.78)$ \\
\hline Unknown & 126 & & \\
\hline \multicolumn{4}{|l|}{$\begin{array}{l}\text { Blood alcohol concentration } \\
(\mathrm{g} / \mathrm{dl})\end{array}$} \\
\hline - & 915 & 174.6 & 1.00 (Reference) \\
\hline $0.01-0.07$ & 46 & 235.9 & $1.36(1.01,1.82)$ \\
\hline$\geq 0.08$ & 78 & 199.5 & $1.14(0.90,1.44)$ \\
\hline \multicolumn{4}{|l|}{$\begin{array}{l}\text { DWI conviction within past } \\
3 \text { years }^{c}\end{array}$} \\
\hline No & 1,023 & 178.2 & 1.00 (Reference) \\
\hline Yes & 8 & 258.1 & $1.45(0.72,2.90)$ \\
\hline Unknown & 57 & & \\
\hline \multicolumn{4}{|l|}{ Crash within past 3 years } \\
\hline No & 908 & 180.3 & 1.00 (Reference) \\
\hline Yes & 115 & 190.4 & $1.06(0.87,1.28)$ \\
\hline Unknown & 189 & & \\
\hline \multicolumn{4}{|l|}{ Day of crash } \\
\hline Monday-Thursday & 601 & 172.5 & 1.00 (Reference) \\
\hline Friday-Sunday & 438 & 186.9 & $1.08(0.96,1.23)$ \\
\hline \multicolumn{4}{|l|}{ Time of crash } \\
\hline Day (7:00am-6:59pm) & 831 & 174.6 & 1.00 (Reference) \\
\hline Night (7:00pm-6:59am) & 203 & 194.8 & $1.12(0.96,1.30)$ \\
\hline Unknown & 27 & & \\
\hline
\end{tabular}




\begin{tabular}{|c|c|c|c|}
\hline \multicolumn{4}{|l|}{$\begin{array}{l}\text { Number of vehicles } \\
\text { involved }\end{array}$} \\
\hline 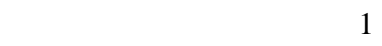 & 428 & 195.4 & $1.16(1.03,1.32)$ \\
\hline$\geq 2$ & 611 & 168.0 & 1.00 (Reference) \\
\hline \multicolumn{4}{|l|}{$\begin{array}{l}\text { Weather conditions at time } \\
\text { of crash }\end{array}$} \\
\hline Clear & 937 & 180.1 & 1.00 (Reference) \\
\hline Adverse & 102 & 163.5 & $0.91(0.74,1.11)$ \\
\hline \multicolumn{4}{|l|}{ Survival time after crash } \\
\hline Death within 1 hour & 553 & 174.7 & 1.00 (Reference) \\
\hline Death beyond 1 hour & 452 & 187.2 & $1.07(0.95,1.21)$ \\
\hline Unknown & 248 & & \\
\hline \multicolumn{4}{|l|}{ Year of crash } \\
\hline 2008 & 166 & 135.8 & 1.00 (Reference) \\
\hline 2009 & 191 & 166.5 & $1.23(1.00,1.51)$ \\
\hline 2010 & 194 & 168.1 & $1.24(1.01,1.52)$ \\
\hline 2011 & 243 & 208.2 & $1.53(1.26,1.87)$ \\
\hline 2012 & 245 & 215.3 & $1.58(1.30,1.93)$ \\
\hline
\end{tabular}

a:States included in this analysis are AK, AZ, CA, CO, FL, HI, IL, IN, KY, MD, MA, MI, MN, NV, NH ,NJ, NM, NY, ND, OH, PA, RI, SC, VT, VA, WA, WV, WY, and Washington, DC.

b: $\mathrm{CI}=$ confidence interval

c: DWI=driving while intoxicated

Table 3. Prevalence rates and ratios of drug involvement in fatally injured drivers

\begin{tabular}{|c|c|c|c|c|c|}
\hline \multirow[t]{2}{*}{ Drug Category } & \multicolumn{2}{|c|}{$\begin{array}{c}\text { Drivers } \geq 65 \text { years } \\
\text { (No. Drivers Tested }=5,828 \text { ) }\end{array}$} & \multicolumn{2}{|c|}{$\begin{array}{c}\text { Drivers } 30-50 \text { years } \\
\text { (No. Drivers } \\
\text { Tested }=15,061)\end{array}$} & \multirow[b]{2}{*}{ Rate ratio ${ }^{\mathrm{a},}\left(95 \% \mathrm{CI}^{\mathrm{b}}\right)$} \\
\hline & No. Positive & rate $/ 1,000$ & $\begin{array}{r}\text { No. } \\
\text { Positive } \\
\end{array}$ & rate/ 1,000 & \\
\hline Any drug usage & 1,039 & 178.3 & 5,140 & 341.3 & $0.53(0.50,0.57)$ \\
\hline $\begin{array}{l}\text { Multiple drug groups } \\
\text { detected }\end{array}$ & 238 & 40.8 & 1,524 & 101.2 & $0.43(0.39,0.48)$ \\
\hline Drug and alcohol use & 124 & 21.2 & 2,861 & 172.7 & $0.12(0.10,0.15)$ \\
\hline Alcohol use only & 462 & 79.3 & 4,508 & 299.3 & $0.21(0.19,0.24)$ \\
\hline
\end{tabular}

a: Rates and ratios were attained through Poisson regression with robust error variance and controlled for the random effects of state

b: $\mathrm{CI}=$ confidence interval 
Table 4. Proportions of broad drug categories and specific drugs identified in fatally injured drivers

\begin{tabular}{|c|c|c|c|c|}
\hline \multirow[t]{2}{*}{ Drug Category or Type } & \multicolumn{2}{|c|}{$\begin{array}{l}\text { Drivers } \geq 65 \text { years old } \\
(\text { No. Positive }=1,039)\end{array}$} & \multicolumn{2}{|c|}{$\begin{array}{l}\text { Drivers } 30-50 \text { years old } \\
(\text { No. Positive }=5,140)\end{array}$} \\
\hline & No. Positive & Proportion $^{\mathrm{a}}(\%)$ & No. Positive & Proportion $(\%)$ \\
\hline \multicolumn{5}{|l|}{ Broad drug categories } \\
\hline Others & 451 & 43.4 & 917 & 17.8 \\
\hline Narcotics & 387 & 37.2 & 1,312 & 25.5 \\
\hline Depressants & 330 & 31.8 & 1,226 & 23.9 \\
\hline Cannabinoid & 66 & 6.4 & 1,778 & 34.6 \\
\hline Stimulants & 57 & 5.5 & 1,564 & 30.4 \\
\hline \multicolumn{5}{|l|}{ Specific drugs } \\
\hline Benzodiazepines & 271 & 26.1 & 1,098 & 21.4 \\
\hline Hydrocodone/Oxycodone & 184 & 17.7 & 842 & 16.4 \\
\hline Barbiturates & 29 & 2.8 & 75 & 1.5 \\
\hline Cocaine & 19 & 1.8 & 896 & 17.4 \\
\hline Methadone & 15 & 1.4 & 200 & 3.9 \\
\hline
\end{tabular}

a: Proportions will not equal $100 \%$ as drivers may have tested positive for multiple drugs 
Table 5a. Most commonly encountered broad drug categories and combinations identified in fatally injured drivers

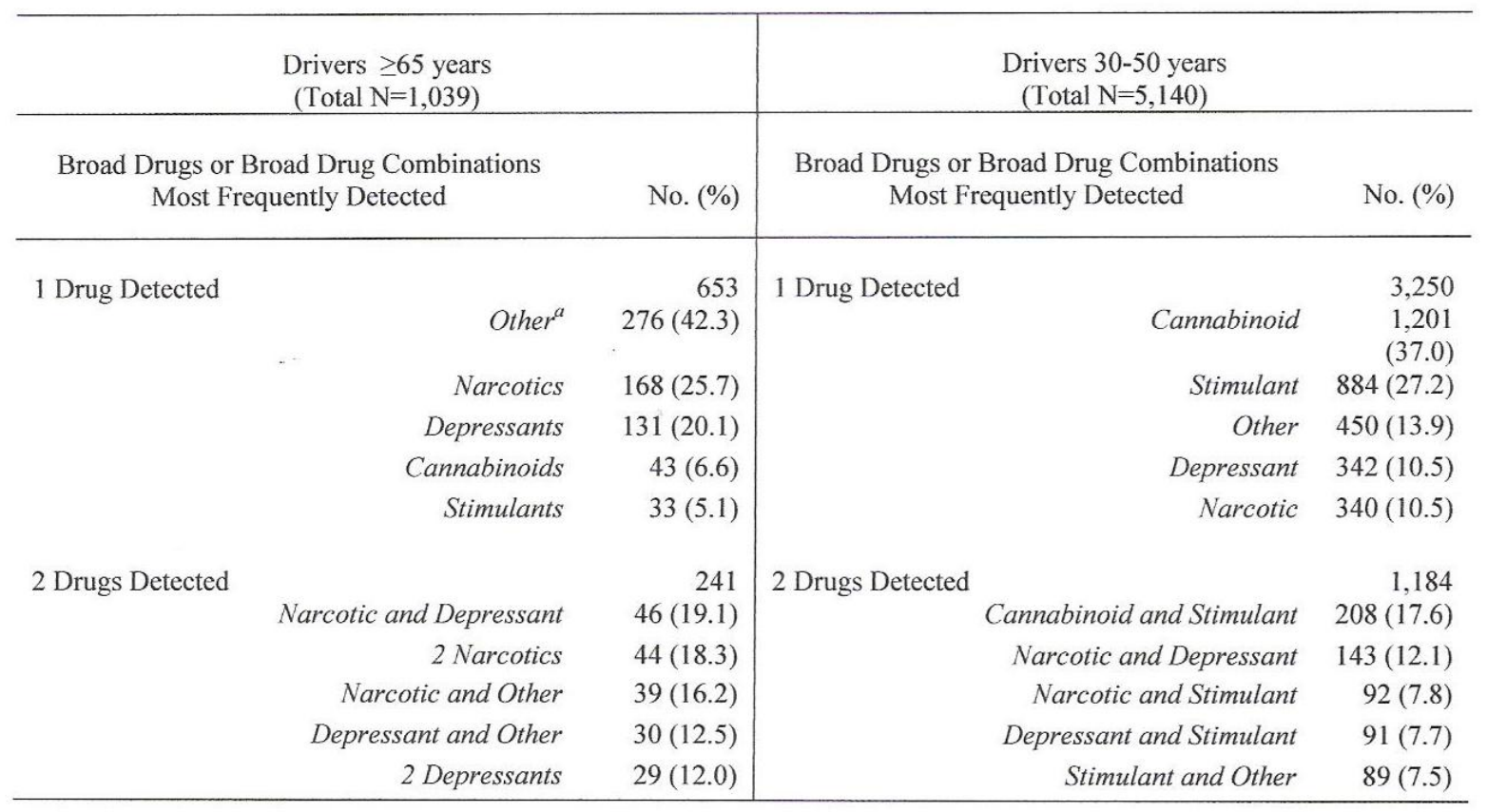

a: Other indicates that the drug(s) detected was not classified as a narcotic, depressant, stimulant, hallucinogen, cannabinoid, phencyclidine, anabolic steroid, or inhalant.

Table 5b. Most commonly encountered specific drugs and specific drug combinations identified in fatally injured drivers

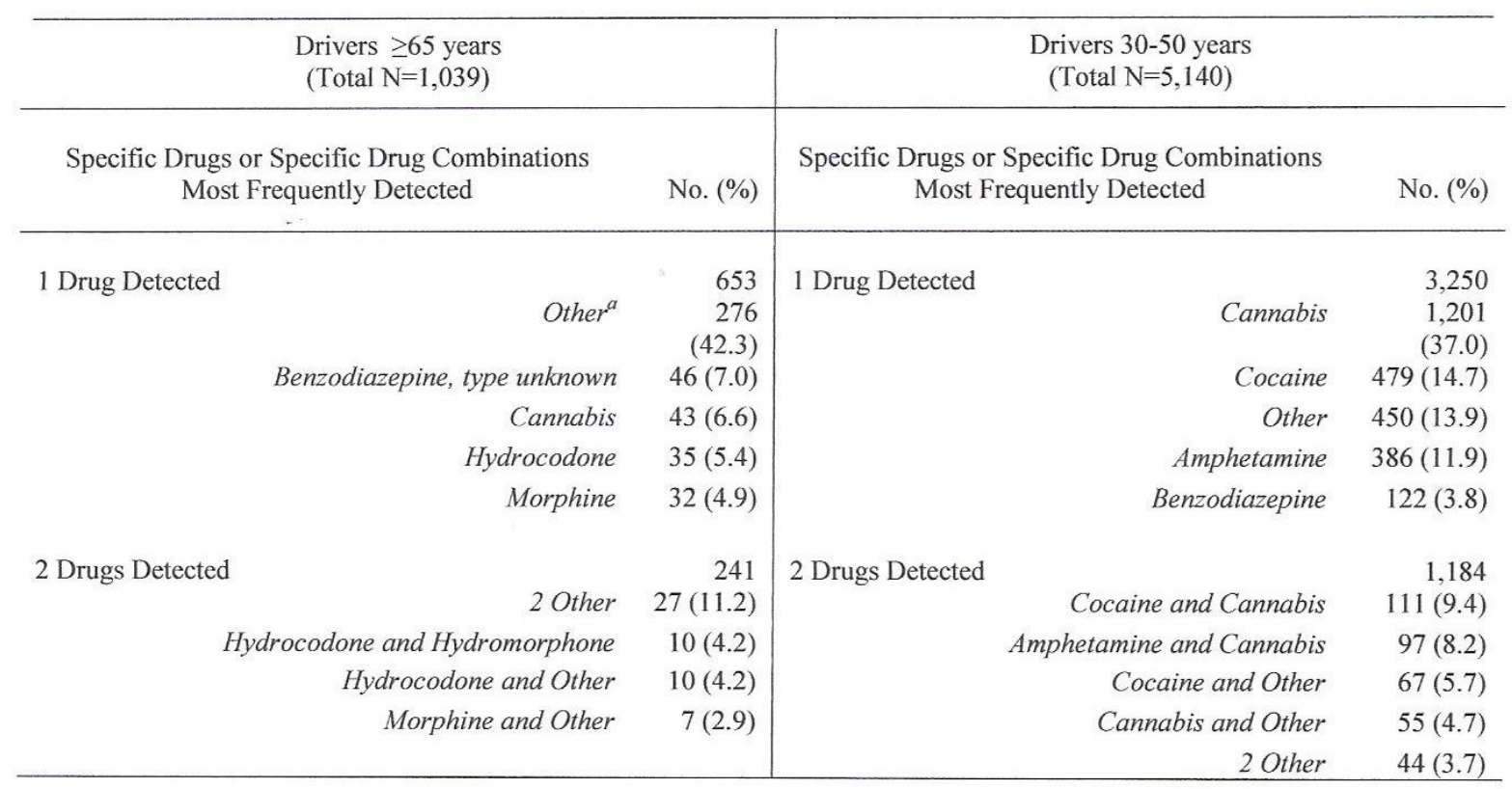

a: Other indicates that the drug(s) detected was not classified as a narcotic, depressant, stimulant, hallucinogen, cannabinoid, phencyclidine, anabolic steroid, or inhalant. 


\section{Chapter 4}

Table 1. Demographic characteristics of case

\begin{tabular}{|c|c|c|c|}
\hline \multirow[t]{2}{*}{ Characteristic } & & \multicolumn{2}{|c|}{$\begin{array}{c}\text { Case Visits } \\
(\mathrm{N}=611)\end{array}$} \\
\hline & & $\mathrm{N}$ & $(\%)$ \\
\hline \multicolumn{4}{|l|}{ Age (years) } \\
\hline & $65-69$ & 225 & $(36.8)$ \\
\hline & $70-79$ & 252 & $(41.2)$ \\
\hline & $80-89$ & 119 & $(19.5)$ \\
\hline & $\geq 90$ & 15 & $(2.5)$ \\
\hline \multicolumn{4}{|l|}{ Gender } \\
\hline & Male & 330 & $(54.0)$ \\
\hline & Female & 281 & $(46.0)$ \\
\hline \multicolumn{4}{|l|}{ Race } \\
\hline & White & 560 & (97.0) \\
\hline & Other & 17 & (3.0) \\
\hline & Missing & 34 & \\
\hline \multicolumn{4}{|c|}{ Day of admittance } \\
\hline & Mon-Thurs & 368 & $(60.2)$ \\
\hline & Fri-Sun & 243 & $(39.8)$ \\
\hline \multicolumn{4}{|c|}{ Time of admittance } \\
\hline & 7:00AM-6:59PM & 462 & (75.7) \\
\hline & 7:00PM-6:59AM & 148 & (24.3) \\
\hline \multicolumn{4}{|c|}{ Season of admittance } \\
\hline & Winter & 133 & $(21.8)$ \\
\hline & Spring & 189 & (30.9) \\
\hline & Summer & 147 & $(24.1)$ \\
\hline & Fall & 142 & $(23.2)$ \\
\hline \multicolumn{4}{|c|}{ Year of admittance } \\
\hline & 2009 & 102 & (16.7) \\
\hline & 2010 & 87 & $(14.2)$ \\
\hline & 2011 & 89 & (14.6) \\
\hline & 2012 & 154 & $(25.2)$ \\
\hline & 2013 & 110 & $(18.0)$ \\
\hline & 2014 & 69 & $(11.3)$ \\
\hline \multicolumn{4}{|c|}{ Method of transport } \\
\hline & Ambulance & 248 & $(40.6)$ \\
\hline & Helicopter & 62 & $(10.2)$ \\
\hline & Self & 37 & $(6.1)$ \\
\hline & Other/Unknown & 264 & $(43.2)$ \\
\hline \multicolumn{4}{|c|}{ Treatment location } \\
\hline & Trauma & 135 & $(22.1)$ \\
\hline & Emergency \& Urgent Care & 476 & $(77.9)$ \\
\hline \multicolumn{4}{|l|}{ Injury severity } \\
\hline & Minor & 29 & $(9.0)$ \\
\hline & Moderate & 43 & (13.4) \\
\hline & Severe & 250 & (77.6) \\
\hline \multirow{2}{*}{ Insurance statu } & Unknown & 289 & \\
\hline & Government & 331 & $(54.2)$ \\
\hline
\end{tabular}




$\begin{array}{rrr}\text { Private } & 181 & (29.6) \\ \text { None } & 30 & (4.9) \\ \text { Other/Unknown } & 69 & (11.3)\end{array}$

County of residence

$\begin{array}{rrr}\text { Monongalia } & 92 & (15.4) \\ \text { Other } & 506 & (84.6) \\ \text { Missing } & 13 & \end{array}$

State of residence

$\begin{array}{rrr}\text { WV } & 491 & (80.4) \\ \text { Other/Missing } & 120 & (19.6) \\ & & \\ \text { Employed } & 74 & (12.4) \\ \text { Retired } & 523 & (87.6) \\ \text { Missing } & 14 & \\ & & \\ 0 & 108 & (17.6) \\ 1-3 & 314 & (51.4) \\ \geq 4 & 189 & (31.0)\end{array}$

Average length of stay (days \pm SD) $\quad 1.9 \pm 4.9$

Drugs detected

\begin{tabular}{lrrr} 
& Yes & 61 & $(31.4)$ \\
& No & 133 & $(68.6)$ \\
Missing & 417 & \\
Alcohol detected & & & \\
& Yes & 12 & $(4.5)$ \\
& No & 257 & $(95.5)$ \\
& Missing & 342 & \\
\hline
\end{tabular}

Table 2. Most common drugs identified in cases via laboratory testing

\begin{tabular}{lcc}
\hline Drug & $\begin{array}{c}\text { Cases testing } \\
\text { positive } \\
\mathrm{N}(\%)\end{array}$ & $\begin{array}{c}\text { Cases testing positive who have } \\
\text { prescriptions for the identified } \\
\text { drug } \\
\mathrm{N}(\%)\end{array}$ \\
\hline Total cases tested & 194 & \\
Benzodiazepines & $33(17.0)$ & $16(48.5)$ \\
Opiates & $30(15.5)$ & $15(50.0)$ \\
\hline
\end{tabular}


Table 3. Most frequently identified broad therapeutic groups, specific medications, and combinations among cases up to 365 days before collision

\begin{tabular}{|c|c|c|}
\hline \multirow[t]{2}{*}{$\begin{array}{l}\text { Total cases } \\
(\mathrm{N}=611) \\
\text { Broad therapeutic groups }\end{array}$} & \multicolumn{2}{|c|}{$\begin{array}{c}\text { Number of cases } \\
\text { taking these } \\
\text { drugs } \\
\mathrm{N}(\%)\end{array}$} \\
\hline & & \\
\hline Analgesics & 82 & (13.4) \\
\hline Cardiovascular & 81 & $(13.3)$ \\
\hline Gastrointestinal & 74 & $(12.1)$ \\
\hline Psychotherapeutics & 48 & (7.9) \\
\hline \multicolumn{3}{|l|}{ Specific medications } \\
\hline Metoprolol & 38 & $(6.2)$ \\
\hline Aspirin & 33 & $(5.4)$ \\
\hline Esomeprazole & 30 & (4.9) \\
\hline Lisinopril & 27 & $(4.4)$ \\
\hline Furosemide & 21 & $(3.4)$ \\
\hline \multicolumn{3}{|l|}{ Combinations } \\
\hline Oxycodone and Acetaminophen & 20 & $(3.3)$ \\
\hline
\end{tabular}


Table 4. The risk of involvement in a motor vehicle collision by medication exposure

\begin{tabular}{|c|c|c|c|c|c|}
\hline \multirow[b]{2}{*}{ Medication } & \multicolumn{5}{|c|}{ 1:4 Matched control periods ${ }^{a}$} \\
\hline & $\mathrm{N}$ & \multicolumn{2}{|c|}{$\begin{array}{c}\text { Model } 1 \\
\text { OR }(95 \% \text { CI })\end{array}$} & \multicolumn{2}{|c|}{$\begin{array}{c}\text { Model } 2 \\
\text { OR }(95 \% \text { CI })\end{array}$} \\
\hline \multicolumn{6}{|l|}{ Anticholesteremic } \\
\hline Simvastatin & 12 & 1.00 & $(0.12,8.17)$ & 0.42 & $(0.03,07.23)$ \\
\hline \multicolumn{6}{|l|}{ Anticoagulants } \\
\hline Clopidogrel & 10 & 10.73 & $(1.19,96.67)$ & 7.62 & $(0.48,122.10)$ \\
\hline Warfarin & 10 & 0.72 & $(0.06,9.04)$ & 0.30 & $(0.08,1.22)$ \\
\hline \multicolumn{6}{|l|}{ Anticonvulsants } \\
\hline Gabapentin & 15 & 2.69 & $(0.59,12.32)$ & 1.32 & $(0.24,7.17)$ \\
\hline \multicolumn{6}{|l|}{ Antidepressants } \\
\hline Citalopram & 10 & 3.01 & $(0.31,29.65)$ & 3.21 & $(0.24,42.50)$ \\
\hline \multicolumn{6}{|l|}{ Antihyperglycemics } \\
\hline Insulin & 12 & 15.76 & $(1.78,139.61)$ & 2.63 & $(0.14,48.72)$ \\
\hline \multicolumn{6}{|l|}{ Antihypertensive } \\
\hline Furosemide & 12 & 0.50 & $(0.08,3.22)$ & 0.81 & $(0.06,10.62)$ \\
\hline Hydrochlorothiazide & 13 & 12.35 & $(1.35,113.06)$ & 15.01 & $(0.76,296.60)$ \\
\hline Lisinopril & 25 & 1.56 & $(0.43,5.69)$ & 0.27 & $(0.05,1.60)$ \\
\hline Metoprolol & 29 & 5.29 & $(1.31,21.38)$ & 1.16 & $(0.23,5.79)$ \\
\hline \multicolumn{6}{|l|}{ Muscle Relaxants } \\
\hline Albuterol & 11 & 0.44 & $(0.06,3.20)$ & 0.25 & $(0.02,3.34)$ \\
\hline \multicolumn{6}{|l|}{ Narcotic Analgesics } \\
\hline Hydrocodone & 15 & 1.32 & $(0.36,4.92)$ & 0.37 & $(0.04,3.79)$ \\
\hline Tramadol & 11 & 10.56 & $(1.17,95.51)$ & 11.41 & $(1.27,102.15)$ \\
\hline \multicolumn{6}{|l|}{ Sleep Medications } \\
\hline Zolpidem & 10 & 4.20 & $(0.73,24.13)$ & 1.42 & $(0.66,3.00)$ \\
\hline \multicolumn{6}{|l|}{ Steroids } \\
\hline Fluticasone & 11 & 0.56 & $(0.08,3.89)$ & 0.41 & $(0.04,4.85)$ \\
\hline Prednisone & 12 & 0.12 & $(0.01,1.07)$ & 0.19 & $(0.02,1.82)$ \\
\hline \multicolumn{6}{|l|}{ Vasodilators } \\
\hline Nitroglycerin & 12 & 2.54 & $(0.42,15.23)$ & 1.27 & $(0.07,23.82)$ \\
\hline \multicolumn{6}{|l|}{ Other Drugs } \\
\hline Alendronate & 10 & 1.15 & $(0.22,6.04)$ & 0.13 & $(0.01,1.78)$ \\
\hline \multicolumn{6}{|l|}{ Combination Drugs } \\
\hline Oxycodone and Acetaminophen & 16 & 0.61 & $(0.18,2.00)$ & 0.17 & $(0.02,1.63)$ \\
\hline
\end{tabular}

a: Conditional logistic regression was used to calculate the odds ratios and $95 \%$ CI. Each case's medication exposure during the 14 day risk period immediately before the crash was matched to four separate control periods up to one year before the collision to assess if medication use during the risk period was associated with an increase of motor vehicle collision compared to control periods. Model 1 is the crude estimate (i.e. unadjusted) while Model 2 was adjusted for the number of medications a person was taking at each case and control period. 
Table 5. The risk of involvement in a motor vehicle collision by medication exposure categorized by pharmaceutical sub-class

\begin{tabular}{|c|c|c|c|c|c|}
\hline \multirow[b]{2}{*}{ Medication sub-class } & \multirow[b]{2}{*}{$\mathrm{N}$} & \multicolumn{4}{|c|}{ 1:4 Matched control periods ${ }^{\mathrm{a}}$} \\
\hline & & Odds & $\begin{array}{l}\text { odel } 1 \\
\text { tio }(95 \% \text { CI) }\end{array}$ & Odds & $\begin{array}{l}\text { Model } 2 \\
\text { Ratio }(95 \% \mathrm{CI})\end{array}$ \\
\hline Anticholesteremics & 31 & 1.50 & $(0.44,5.18)$ & 0.42 & $(0.08,2.21)$ \\
\hline Anticoagulants & 20 & 3.59 & $(0.84,15.28)$ & 2.29 & $(0.35,15.19)$ \\
\hline Antidepressants & 30 & 2.05 & $(0.59,7.16)$ & 0.50 & $(0.09,2.67)$ \\
\hline Antihyperglycemics & 22 & 15.36 & $(1.79,132.0)$ & 2.24 & $(0.17,29.84)$ \\
\hline Antihypertensives & 39 & 3.32 & $(1.15,9.62)$ & 1.24 & $(0.29,5.32)$ \\
\hline Benzodiazepines & 21 & 1.96 & $(0.58,6.62)$ & 0.71 & $(0.15,3.34)$ \\
\hline Narcotic Analgesics & 41 & 1.56 & $(0.72,3.39)$ & 0.94 & $(0.32,2.75)$ \\
\hline
\end{tabular}

a: Conditional logistic regression was used to calculate the odds ratios and 95\% CI. Each case's medication exposure during the 14 day risk period immediately before the crash was matched to four separate control periods up to one year before the collision to assess if medication use during the risk period was associated with an increase of motor vehicle collision compared to control periods. Model 1 is the crude estimate (i.e. unadjusted) while Model 2 was adjusted for the number of medications a person was taking at each case and control period. 